\title{
Toward a General Route to the Eunicellin Diterpenes: The Asymmetric Total Synthesis of Deacetoxyalcyonin Acetate
}

Gary A. Molander *, David J. St. Jean, Jr. and Julia Haas

Roy and Diana Vagelos Laboratories, Department of Chemistry, University of Pennsylvania, Philadelphia, Pennsylvania 19104-6323

Cylcobutanone 4: $(R)-\alpha$-Phellandrene (121 g, $617 \mathrm{mmol}, 70 \%$ pure) along with $300 \mathrm{~mL}$ of toluene was heated to reflux. To this $67.8 \mathrm{~g}(668 \mathrm{mmol})$ of $\mathrm{NEt}_{3}$ and $55.7 \mathrm{~g}$ of methoxyacetyl chloride $(514 \mathrm{mmol})$ were added slowly and simultaneously, maintaining approximately a $10 \%$ excess of $\mathrm{NEt}_{3}$. After the addition was complete, the reaction mixture was heated at reflux for an additional $30 \mathrm{~min}$, cooled to rt, diluted with water, and extracted with EtOAc $(3 \times 750 \mathrm{~mL})$. The combined organic extracts were dried with $\mathrm{Na}_{2} \mathrm{SO}_{4}$, filtered and concentrated. The crude product was purified by column chromatography twice $\left(\mathrm{SiO}_{2}, 5 \% \mathrm{EtOAc} /\right.$ petroleum ether, $\left.1 \% \mathrm{NEt}_{3}\right)$ to give $30.0 \mathrm{~g}\left(25 \%, 91 \%\right.$ pure by GC) of 4 as a colorless oil. ${ }^{1} \mathrm{H} \mathrm{NMR}\left(500 \mathrm{MHz}, \mathrm{CDCl}_{3}\right) \delta 5.56-$ $5.43(\mathrm{~m}, 1 \mathrm{H}), 4.53-4.49(\mathrm{~m}, 1 \mathrm{H}), 3.50(\mathrm{~s}, 3 \mathrm{H}), 3.25-3.22(\mathrm{~m}, 1 \mathrm{H}), 2.99-2.92(\mathrm{~m}, 1 \mathrm{H}), 2.21-2.12$ (m, 1H), 1.94-1.87 (m, 1H), 1.86-1.81 (m, 1H), $1.76(\mathrm{~s}, 3 \mathrm{H}), 1.54-1.49(\mathrm{~m}, 1 \mathrm{H}), 0.86-0.84(\mathrm{~m}$, $6 \mathrm{H}) ;{ }^{13} \mathrm{C}$ NMR $\left(125 \mathrm{MHz}, \mathrm{CDCl}_{3}\right) \delta 209.8,130.8,123.0,88.6,59.6,52.6,34.47,33.7,30.4$, 24.4, 23.8, 21.0, 20.7; IR (neat) 2960, 1777, $1459 \mathrm{~cm}^{-1}$; HRMS calcd for $\mathrm{C}_{13} \mathrm{H}_{20} \mathrm{O}_{2} \mathrm{Na}(\mathrm{M}+\mathrm{Na})^{+}$ 231.1361 , found 231.1358 .

Mixed acetal 5: $4.3 \mathrm{~g}$ ( $21 \mathrm{mmol}, 90 \%$ purity by GC) of cyclobutanone 4 was dissolved in 250 $\mathrm{mL}$ of $\mathrm{CH}_{2} \mathrm{Cl}_{2}$, and acetic acid $(4.3 \mathrm{~mL})$ was added. The solution was degassed by two freeze/pump/thaw cycles and placed in a degassed photochemical reactor equipped with a borosilicate glass immersion well and a $450 \mathrm{~W}$ medium pressure UV lamp. The solution was irradiated at $10{ }^{\circ} \mathrm{C}$ for $42 \mathrm{~h}$, quenched with $13.0 \mathrm{~g}$ of solid $\mathrm{K}_{2} \mathrm{CO}_{3}$, filtered, and concentrated. 
The residue was purified by flash chromatography $\left(\mathrm{SiO}_{2}, 5 \% \mathrm{EtOAc} /\right.$ petroleum ether, $\left.1 \% \mathrm{NEt}_{3}\right)$ to give $4.30 \mathrm{~g}$ of 5 ( $86 \%$ based on starting material purity, $90 \%$ pure by GC). ${ }^{1} \mathrm{H}$ NMR (500 $\left.\mathrm{MHz}, \mathrm{CDCl}_{3}\right) \delta 6.37(\mathrm{~s}, 1 \mathrm{H}), 5.61-5.58(\mathrm{~m}, 1 \mathrm{H}), 5.13(\mathrm{~d}, J=6.0 \mathrm{~Hz}, 1 \mathrm{H}), 3.38(\mathrm{~s}, 3 \mathrm{H}), 2.85(\mathrm{~m}$, 1H), 2.12-1.94 (m, 5H), 1.72-1.64 (m, 5H), $0.92(\mathrm{~d}, J=6.9 \mathrm{~Hz}, 3 \mathrm{H}), 0.92-0.80(\mathrm{~m}, 1 \mathrm{H}), 0.76(\mathrm{~d}$, $J=6.9 \mathrm{~Hz}, 3 \mathrm{H}) ;{ }^{13} \mathrm{C} \mathrm{NMR}\left(125 \mathrm{MHz}, \mathrm{CDCl}_{3}\right) \delta 170.1,128.7,123.8,106.2,100.0,56.3,45.8$, 45.0, 36.4, 27.8, 23.1, 22.1, 21.3, 21.2, 15.1; IR (neat) 2958, 1751, 1442, $1368 \mathrm{~cm}^{-1}:$ HRMS calcd for $\mathrm{C}_{13} \mathrm{H}_{21} \mathrm{O}_{2}(\mathrm{M}-\mathrm{OAc})^{+}$209.1542, found 209.1534.

Annulation product 6: To a flask containing $9.4 \mathrm{~g}(35.0 \mathrm{mmol}, 80 \%$ pure by GC) of 5 was added $12.06 \mathrm{~g}$ (35.0 mmol) of 1-methoxy-1,3-bis(triethylsilanyloxy)buta-1,3-diene ${ }^{1}$ followed by $200 \mathrm{~mL}$ of $\mathrm{CH}_{2} \mathrm{Cl}_{2}$. This solution was then cooled to $-80{ }^{\circ} \mathrm{C}$ and a solution of $15.9 \mathrm{~g}$ of $\mathrm{TiCl}_{4}$ in $150 \mathrm{~mL}$ of $\mathrm{CH}_{2} \mathrm{Cl}_{2}$ was then added over $1.5 \mathrm{~h}$. The resulting red solution was stirred for $10 \mathrm{~h}$ at $80{ }^{\circ} \mathrm{C}$ after which $100 \mathrm{~mL}$ of saturated aqueous $\mathrm{NaHCO}_{3}$ was added. After warming to rt, the biphasic solution was extracted with $\mathrm{CH}_{2} \mathrm{Cl}_{2}(4 \times 100 \mathrm{~mL})$, dried, filtered, and concentrated to give a dark yellow oil. Purification twice by MPLC $\left(\mathrm{SiO}_{2}, 3 \%\right.$ EtOAc/petroleum ether) yielded $3.7 \mathrm{~g}$ of 6 (43\% based on purity of starting material) as tan crystals. ${ }^{2} \mathrm{mp} 70{ }^{\circ} \mathrm{C} ;{ }^{1} \mathrm{H}$ NMR (500 $\left.\mathrm{MHz}, \mathrm{CDCl}_{3}\right) \delta 11.60(\mathrm{br}, 1 \mathrm{H}), 5.53-5.47(\mathrm{~m}, 1 \mathrm{H}), 4.69(\mathrm{~s}, 1 \mathrm{H}), 4.19-4.12(\mathrm{~m}, 1 \mathrm{H}), 3.78(\mathrm{~s}, 3 \mathrm{H})$, 2.81-2.60 (m, 1H), $2.54(\mathrm{~m}, 1 \mathrm{H}), 2.39-2.31(\mathrm{~m}, 1 \mathrm{H}), 2.12-1.91(\mathrm{~m}, 3 \mathrm{H}), 1.72(\mathrm{~s}, 3 \mathrm{H}), 1.44-1.32$ $(\mathrm{m}, 2 \mathrm{H}), 0.90(\mathrm{~d}, J=6.4 \mathrm{~Hz}, 3 \mathrm{H}), 0.82(\mathrm{~d}, J=6.5 \mathrm{~Hz}, 3 \mathrm{H}) ;{ }^{13} \mathrm{C} \mathrm{NMR}\left(125 \mathrm{MHz}, \mathrm{CDCl}_{3}\right) \delta$ $170.2,169.1,134.0,122.3,103.8,80.9,75.0,51.7,51.5,45.0,42.7,37.4,29.1,23.0,21.7,21.5$, 20.8; IR (neat) 2955, 1672, $1602 \mathrm{~cm}^{-1}$; HRMS calcd for $\mathrm{C}_{17} \mathrm{H}_{25} \mathrm{O}_{4}(\mathrm{M}+\mathrm{H})^{+} 293.1753$, found 293.1756; $[\alpha]_{\mathrm{D}}^{20}=-68.50\left(c 0.96, \mathrm{CHCl}_{3}\right)$. 
Methyl Ketones 7 and 8: A round-bottomed flask containing $4.50 \mathrm{~g}(106.0 \mathrm{mmol})$ of dry $\mathrm{LiCl}$ was flame-dried under vacuum and then cooled to rt. $1.25 \mathrm{~g}(4.28 \mathrm{mmol})$ of 6 in $50 \mathrm{~mL}$ of THF was then added via cannula. This slurry was cooled to $-78{ }^{\circ} \mathrm{C}$, after which $4.45 \mathrm{~mL}$ of $n-\mathrm{BuLi}$ (2.38 $\mathrm{M}$ in hexanes, $10.50 \mathrm{mmol}$ ) was added dropwise. After the addition was complete, the dark yellow solution was stirred at $-78{ }^{\circ} \mathrm{C}$ for $1 \mathrm{~h}$. Iodomethane $(1.05 \mathrm{~g}, 7.40 \mathrm{mmol})$ was added over several minutes at $-78{ }^{\circ} \mathrm{C}$. This solution was stirred for 15 min at $-78{ }^{\circ} \mathrm{C}$, quenched with saturated aqueous $\mathrm{NH}_{4} \mathrm{Cl}$, and allowed to warm to rt. The organics were repeatedly extracted with $\mathrm{Et}_{2} \mathrm{O}(3 \times 30 \mathrm{~mL})$, dried with $\mathrm{MgSO}_{4}$, filtered, and concentrated to give a tan colored solid. $1.0 \mathrm{~g}(20.35 \mathrm{mmol})$ of $\mathrm{LiCl}, 300 \mu \mathrm{L}$ of $\mathrm{H}_{2} \mathrm{O}$, and $80 \mathrm{~mL}$ of DMSO were then added. This solution was heated to $130{ }^{\circ} \mathrm{C}$ where in the solution slowly darkened. After heating at $130{ }^{\circ} \mathrm{C}$ for $3 \mathrm{~h}$, the solution was cooled to rt, diluted with ether and repeatedly washed with brine $(6 \times 50$ $\mathrm{mL}$ ). The organic extracts were dried with $\mathrm{MgSO}_{4}$, filtered, and concentrated to give a dark yellow oil which was purified by column chromatography $\left(\mathrm{SiO}_{2}, 8 \%\right.$ EtOAc/petroleum ether) to yield $383 \mathrm{mg}$ of $\mathbf{8}$ and $117 \mathrm{mg}$ of 7 (50\% combined yield over 2 steps).

Methyl ketone 7: ${ }^{1} \mathrm{H}$ NMR $\left(500 \mathrm{MHz}, \mathrm{CDCl}_{3}\right) \delta 5.57(\mathrm{~m}, 1 \mathrm{H}), 4.48(\mathrm{~d}, J=4.89 \mathrm{~Hz}, 1 \mathrm{H}), 4.04$ (s, 1H), 2.88-2.83 (dd, $J=5.3,15.9 \mathrm{~Hz}, 1 \mathrm{H}), 2.38-2.15(\mathrm{~m}, 4 \mathrm{H}), 1.98-1.95(\mathrm{~m}, 1 \mathrm{H}) 1.77-1.58$ (m, 5H), $1.35-1.26(\mathrm{~m}, 4 \mathrm{H}), 0.94-0.86(\mathrm{~d}, J=6.8 \mathrm{~Hz}, 3 \mathrm{H}), 0.79(\mathrm{~d}, J=6.8 \mathrm{~Hz}, 3 \mathrm{H}) ;{ }^{13} \mathrm{C}$ NMR $\left(125 \mathrm{MHz}, \mathrm{CDCl}_{3}\right) \delta 211.5,132.4,123.04,84.9,78.6,52.6,47.0,46.9,45.5,41.4,28.9,22.4$, 22.2, 21.6, 18.0, 16.9; IR (neat) 2959, 1716, $1456 \mathrm{~cm}^{-1}$; HRMS calcd for $\mathrm{C}_{16} \mathrm{H}_{24} \mathrm{O}_{2}(\mathrm{M})^{+}$ 248.1776, found 248.1783; $[\alpha]^{20}=-109.28\left(c 0.37, \mathrm{CHCl}_{3}\right)$. 
Methyl ketone 8: $\mathrm{mp} 49{ }^{\circ} \mathrm{C} ;{ }^{1} \mathrm{H}$ NMR $\left(500 \mathrm{MHz}, \mathrm{CDCl}_{3}\right) \delta 5.52(\mathrm{~m}, 1 \mathrm{H}), 4.48(\mathrm{~d}, J=4.7 \mathrm{~Hz}$, $1 \mathrm{H}), 4.13(\mathrm{~d}, J=4.5 \mathrm{~Hz}, 1 \mathrm{H}), 2.74-2.69(\mathrm{~m}, 2 \mathrm{H}), 2.29-2.24(\mathrm{~m}, 2 \mathrm{H}), 2.17-2.14(\mathrm{~m}, 1 \mathrm{H}), 1.94-$ $1.91(\mathrm{~m}, 1 \mathrm{H}), 1.74-1.69(\mathrm{~m}, 1 \mathrm{H}), 1.63(\mathrm{~s}, 3 \mathrm{H}), 1.57-1.55(\mathrm{~m}, 1 \mathrm{H}), 1.24-1.23(\mathrm{~m}, 1 \mathrm{H}), 0.99(\mathrm{~d}, J$ $=7.5 \mathrm{~Hz}, 3 \mathrm{H}), 0.90(\mathrm{~d}, J=6.8 \mathrm{~Hz}, 3 \mathrm{H}), 0.74(\mathrm{~d}, J=7.6 \mathrm{~Hz}, 3 \mathrm{H}) ;{ }^{13} \mathrm{C} \mathrm{NMR}\left(125 \mathrm{MHz}, \mathrm{CDCl}_{3}\right) \delta$ $208.8,132.5,123.0,85.1,79.5,51.7,49.0,47.4,42.0,41.3,28.9,22.3,22.1,21.6,18.1,9.6$; IR (neat) $2958,1714 \mathrm{~cm}^{-1}$; HRMS calcd for $\mathrm{C}_{16} \mathrm{H}_{24} \mathrm{O}_{2}(\mathrm{M})^{+} 248.1776$, found 248.1772; $[\alpha]^{20}=$ $44.40\left(\right.$ c $\left.1.09, \mathrm{CHCl}_{3}\right)$.

Equilibration of ketones 7 and 8: A $50 \mathrm{~mL}$ round-bottom flask was charged with $1.37 \mathrm{~g}$ (5.5 mmol) of 8. To this was added $25 \mathrm{~mL}$ of $\mathrm{MeOH}$ and $1.49 \mathrm{~g}(27.6 \mathrm{mmol})$ of NaOMe. This solution was heated at reflux for $45 \mathrm{~min}$ and quenched by the addition of $\sim 20 \mathrm{~g}$ of $\mathrm{SiO}_{2}$. Evaporation of the solvent followed by column chromatography yielded $1.0 \mathrm{~g}(73 \%)$ of 7 and $370 \mathrm{mg}(27 \%)$ of $\mathbf{8}$.

TBS enol ether 9: A flame dried round-bottomed flask was charged with $1.68 \mathrm{~g}(6.7 \mathrm{mmol})$ of 8 and $6.12 \mathrm{~g}$ (40.6 mmol) of TBSCl. $70 \mathrm{~mL}$ of THF was then added and the solution was cooled to $-78{ }^{\circ} \mathrm{C}$. Under a positive pressure of nitrogen, $\mathrm{KH}(1.62 \mathrm{~g}, 40.6 \mathrm{mmol})$ was then added and the solution was allowed to warm slowly to rt over $4 \mathrm{~h}$, and allowed to stir for an additional $2 \mathrm{~h}$. 25 mL of saturated aqueous $\mathrm{NaHCO}_{3}$ was added and the mixture was extracted with $\mathrm{CH}_{2} \mathrm{Cl}_{2}(4 \times 35$ $\mathrm{mL}$ ), dried with $\mathrm{MgSO}_{4}$, filtered, and concentrated to give an oil. Column chromatography $\left(\mathrm{SiO}_{2}, 2 \% \mathrm{EtOAc} /\right.$ petroleum ether, $\left.1 \% \mathrm{NEt}_{3}\right)$ provided $2.38 \mathrm{~g}(95 \%)$ of pure $9 .{ }^{1} \mathrm{H}$ NMR (500 $\left.\mathrm{MHz}, \mathrm{CDCl}_{3}\right) \delta 5.51(\mathrm{~m}, 1 \mathrm{H}), 4.27(\mathrm{~s}, 1 \mathrm{H}), 4.13(\mathrm{~s}, 1 \mathrm{H}), 2.68(\mathrm{~d}, J=14.0 \mathrm{~Hz}, 1 \mathrm{H}), 2.41(\mathrm{br}, 1 \mathrm{H})$, 2.23-2.19 (m, 1H), 1.92-1.85 (m, 2H), 1.74-1.66 (m, 4H), $1.60(\mathrm{~s}, 3 \mathrm{H}), 1.38-1.34(\mathrm{~m}, 1 \mathrm{H}), 1.24$ 
(m, 1H), 0.98-0.84 (m, 12H), $0.82(\mathrm{~d}, J=6.9 \mathrm{~Hz}, 3 \mathrm{H}), 0.13(\mathrm{~s}, 6 \mathrm{H}) ;{ }^{13} \mathrm{C} \mathrm{NMR}\left(125 \mathrm{MHz}, \mathrm{CDCl}_{3}\right)$

$\delta 139.7,132.9,122.7,115.0,79.5,76.7,49.7,48.7,39.8,39.3,28.6,25.7,22.6,22.6,21.7,18.1$,

15.6, 12.4, -3.8, -3.8; IR (neat) 2956, 2928, $1686 \mathrm{~cm}^{-1}$; HRMS calcd for $\mathrm{C}_{22} \mathrm{H}_{38} \mathrm{O}_{2} \mathrm{Si}(\mathrm{M})^{+}$

362.2641, found 326.2643; $[\alpha]^{20}=-10.97\left(c 0.98, \mathrm{CHCl}_{3}\right)$.

Enone 10: A round-bottomed flask containing $1.08 \mathrm{~g}(3.0 \mathrm{mmol})$ of 9 and $30 \mathrm{~mL}$ of THF was cooled to $-78{ }^{\circ} \mathrm{C}$. To this flask was added a solution of $\mathrm{PhSeCl}(632 \mathrm{mg}, 3.3 \mathrm{mmol})$ in $10 \mathrm{~mL}$ of THF via cannula over $5 \mathrm{~min}$. After stirring for $30 \mathrm{~min}$ at $-78{ }^{\circ} \mathrm{C}$, the flask was warmed to $0{ }^{\circ} \mathrm{C}$ and $961 \mathrm{mg}$ ( $3.9 \mathrm{mmol}, 70 \%$ pure) of $m-\mathrm{CPBA}$ was then added. The solution was allowed to stir for 30 min at $0{ }^{\circ} \mathrm{C}$, then $25 \mathrm{~mL}$ of a $10 \%$ aqueous $\mathrm{Na}_{2} \mathrm{CO}_{3}$ solution was added. The solution was extracted with $\mathrm{CH}_{2} \mathrm{Cl}_{2}(4 \times 20 \mathrm{~mL})$, dried with $\mathrm{MgSO}_{4}$, filtered, and concentrated to give a dark yellow oil. Purification by MPLC $\left(\mathrm{SiO}_{2}, 5 \%\right.$ EtOAc/petroleum ether) provided $554 \mathrm{mg}(74 \%)$ of 10 as a colorless oil. ${ }^{1} \mathrm{H}$ NMR (500 MHz, $\left.\mathrm{CDCl}_{3}\right) \delta 6.00(\mathrm{~s}, 1 \mathrm{H}), 5.54(\mathrm{~m}, 1 \mathrm{H}), 5.15(\mathrm{~s}, 1 \mathrm{H}), 4.79$ (m, 1H), $4.43(\mathrm{~m}, 1 \mathrm{H}), 2.80-2.76(\mathrm{dd}, J=5.3,17.8 \mathrm{~Hz}, 1 \mathrm{H}), 2.47$ (m, 1H), $2.34(\mathrm{~m}, 1 \mathrm{H}), 2.16-$ $2.12(\mathrm{~m}, 1 \mathrm{H}), 1.94-1.87(\mathrm{~m}, 2 \mathrm{H}), 1.67-1.62(\mathrm{~m}, 4 \mathrm{H}), 1.48-1.41(\mathrm{~m}, 1 \mathrm{H}), 0.96(\mathrm{~d}, J=6.9 \mathrm{~Hz}$ $3 \mathrm{H}), 0.81(\mathrm{~d}, J=6.9 \mathrm{~Hz}, 3 \mathrm{H}) ;{ }^{13} \mathrm{C} \mathrm{NMR}\left(125 \mathrm{MHz}, \mathrm{CDCl}_{3}\right) \delta 196.8,146.0,131.7,123.2,119.0$, 81.7, 77.6, 48.7, 48.3, 48.1, 39.1, 28.5, 22.3, 22.2, 21.5, 16.3; IR (neat) 1702, $1623 \mathrm{~cm}^{-1}$; HRMS calcd for $\mathrm{C}_{16} \mathrm{H}_{22} \mathrm{O}_{2}(\mathrm{M}+\mathrm{H})^{+} 247.1698$, found 247.1701; $[\alpha]^{20}{ }_{\mathrm{D}}=-3.90\left(c 10.3, \mathrm{CHCl}_{3}\right)$.

Vinyl triflate 11: $378 \mathrm{mg}$ (2.5 mmol) of cis-1-bromo-2-ethoxyethylene were dissolved in $10 \mathrm{~mL}$ of THF and cooled to $-78{ }^{\circ} \mathrm{C} .3 .40 \mathrm{~mL}(1.5 \mathrm{M}$ in pentane, $5.1 \mathrm{mmol})$ of $t$-BuLi was then added slowly over $1 \mathrm{~h}$ and the solution was allowed to stir for an additional hour at $-78^{\circ} \mathrm{C}$. Under a positive pressure of nitrogen, $258 \mathrm{mg}(1.25 \mathrm{mmol})$ of $\mathrm{CuBr} \cdot \mathrm{SMe}_{2}$ was added and the solution 
was stirred for $2 \mathrm{~h}$ at $-78{ }^{\circ} \mathrm{C}$ until a clear orange solution had formed. A $0.5 \mathrm{M}$ solution of $\mathbf{1 0}$ (234 mg, $1.0 \mathrm{mmol}$ ) in THF was then added dropwise and the resulting yellow mixture was stirred for $1 \mathrm{~h}$ at $-78{ }^{\circ} \mathrm{C}$. After $1 \mathrm{~h}$, a solution of $N$-4-chloropyridyltriflimide $(1.18 \mathrm{~g}, 3.0 \mathrm{mmol})$ in $10 \mathrm{~mL}$ of THF was added and the red solution was stirred for an additional $2 \mathrm{~h}$ at $-78{ }^{\circ} \mathrm{C}$. The reaction was quenched with $10 \mathrm{~mL}$ of saturated aqueous $\mathrm{NH}_{4} \mathrm{Cl}$, and the resulting solution was extracted with $\mathrm{Et}_{2} \mathrm{O}(3 \times 30 \mathrm{~mL})$ and $\mathrm{EtOAc}(2 \times 20 \mathrm{~mL})$. The organic extracts were dried with $\mathrm{Na}_{2} \mathrm{SO}_{4}$, filtered, concentrated, and dissolved in $5 \mathrm{~mL}$ of THF. $2 \mathrm{~mL}$ of $1 \mathrm{M} \mathrm{HCl}$ were added and the resulting suspension was stirred for $36 \mathrm{~h}$ at rt. The reaction mixture was portioned between water $(10 \mathrm{~mL})$ and $\mathrm{Et}_{2} \mathrm{O}(20 \mathrm{~mL})$ and the aqueous layer was extracted with $\mathrm{EtOAc}(2 \mathrm{x}$ $10 \mathrm{~mL}$ ). The organic extracts were washed with saturated aqueous $\mathrm{NaHCO}_{3}$, dried over $\mathrm{Na}_{2} \mathrm{SO}_{4}$, filtered, and concentrated to give a crude oil. Purification by column chromatography $\left(\mathrm{SiO}_{2}, 10 \%\right.$ EtOAc/ petroleum ether) yielded $304 \mathrm{mg}$ ( $71 \%$ over two steps) of $\mathbf{1 1}$ as a clear wax. ${ }^{1} \mathrm{H}$ NMR $\left(500 \mathrm{MHz}, \mathrm{CDCl}_{3}\right) \delta 9.79(\mathrm{~s}, 1 \mathrm{H}), 5.54(\mathrm{~m}, 1 \mathrm{H}), 4.38-4.34(\mathrm{~m}, 2 \mathrm{H}), 2.97-2.93(\mathrm{dd}, J=4.4,16.2$ $\mathrm{Hz}, 1 \mathrm{H}), 2.68-2.58(\mathrm{~m}, 3 \mathrm{H}), 2.46-2.45(\mathrm{br}, 1 \mathrm{H}), 2.34-2.24(\mathrm{~m}, 2 \mathrm{H}), 2.10-2.07$ (d, $J=16.2 \mathrm{~Hz}$, 1H), $1.94-1.83(\mathrm{~m}, 2 \mathrm{H}), 1.70-1.54(\mathrm{~m}, 4 \mathrm{H}), 1.41-1.35(\mathrm{~m}, 1 \mathrm{H}), 0.96(\mathrm{~d}, J=6.9 \mathrm{~Hz}, 3 \mathrm{H}), 0.81(\mathrm{~d}$, $J=6.9 \mathrm{~Hz}, 3 \mathrm{H}) ;{ }^{13} \mathrm{C} \mathrm{NMR}\left(125 \mathrm{MHz}, \mathrm{CDCl}_{3}\right) \delta 199.7,140.5,132.8,131.6,123.3,78.0,77.4$, $50.5,48.4,41.4,39.4,37.2,28.7,22.5,22.3,21.6,19.5,15.7 ;{ }^{19} \mathrm{~F} \mathrm{NMR}\left(300 \mathrm{MHz}, \mathrm{CDCl}_{3}\right) \delta$ 74.8; IR (neat) 1672, $1602 \mathrm{~cm}^{-1}$; HRMS calcd for $\mathrm{C}_{19} \mathrm{H}_{25} \mathrm{O}_{5} \mathrm{~F}_{3} \mathrm{~S}(\mathrm{M})^{+} 422.1375$, found 422.1373; $[\alpha]^{20}=-22.80\left(c 1.20, \mathrm{CHCl}_{3}\right)$.

Allylic alcohols 12 and 13: In a nitrogen filled glove box, a $100 \mathrm{~mL}$ flask was charged with 500 $\mathrm{mg}(4.06 \mathrm{mmol})$ of $\mathrm{CrCl}_{2}$ and $5 \mathrm{mg}(0.039 \mathrm{mmol})$ of $\mathrm{NiCl}_{2}$. After removing the flask from the glove box, $10 \mathrm{~mL}$ of anhydrous DMF was added and the solution was allowed to stir at $\mathrm{rt}$ for $1 \mathrm{~h}$. 
This blue-green solution was cooled to $0{ }^{\circ} \mathrm{C}$ and a solution of $210 \mathrm{mg}(0.47 \mathrm{mmol})$ of 11 in 10 $\mathrm{mL}$ of THF was then added dropwise. The solution was slowly allowed to warm to rt overnight. $3 \mathrm{~mL}$ of a $1 \mathrm{M}$ solution of (DL)-serinate in saturated aqueous $\mathrm{NaHCO}_{3}$ was slowly added. 30 $\mathrm{mL}$ of ether was added and the aqueous layer was further extracted with both $\mathrm{Et}_{2} \mathrm{O}(2 \times 15 \mathrm{~mL})$ and EtOAc $(1 \times 20 \mathrm{~mL})$. The organic extracts were dried with $\mathrm{MgSO}_{4}$, filtered, and concentrated to give a dark colored oil. Purification by gradient column chromatography $\left(\mathrm{SiO}_{2} 25 \% \mathrm{EtOAc} /\right.$ petroleum ether $\rightarrow 50 \% \mathrm{EtOAc} /$ petroleum ether) yielded $35 \mathrm{mg}(27 \%)$ of $\mathbf{1 2}$ and $78 \mathrm{mg}(60 \%)$ of 13.

Alcohol 12: ${ }^{1} \mathrm{H}$ NMR $\left(500 \mathrm{MHz}, \mathrm{CDCl}_{3}\right) \delta 5.50(\mathrm{~s}, 1 \mathrm{H}), 4.64(\mathrm{~s}, 1 \mathrm{H}), 4.35-4.32(\mathrm{br}, 2 \mathrm{H}), 2.56-$ $2.53(\mathrm{~m}, 1 \mathrm{H}), 2.43-2.21(\mathrm{~m}, 5 \mathrm{H}), 2.01-1.87(\mathrm{~m}, 3 \mathrm{H}), 1.70-1.55(\mathrm{~m}, 6 \mathrm{H}), 1.45-1.39(\mathrm{~m}, 1 \mathrm{H})$, $0.93(\mathrm{~d}, J=6.9 \mathrm{~Hz}, 3 \mathrm{H}), 0.77(\mathrm{~d}, J=6.9 \mathrm{~Hz}, 3 \mathrm{H}) ;{ }^{13} \mathrm{C} \mathrm{NMR}\left(125 \mathrm{MHz}, \mathrm{CDCl}_{3}\right) \delta 143.1,132.8$, $132.7,122.5,78.3,77.0,76.8,49.6,49.1,39.0,33.4,32.7,30.1,28.7,22.8,22.6,21.6,15.7$; IR (neat) 3390, 2957, 2923, 2892, 2872, $2849 \mathrm{~cm}^{-1}$; HRMS calcd for $\mathrm{C}_{18} \mathrm{H}_{25} \mathrm{O}_{2}(\mathrm{M}-\mathrm{H})^{+} 273.1855$, found 273.1858; $[\alpha]^{20}{ }_{\mathrm{D}}=-7.71\left(c 0.35, \mathrm{CHCl}_{3}\right)$.

Alcohol 13: $\mathrm{mp} 89-90{ }^{\circ} \mathrm{C} ;{ }^{1} \mathrm{H}$ NMR $\left(500 \mathrm{MHz}, \mathrm{CDCl}_{3}\right) \delta 5.49(\mathrm{~m}, 1 \mathrm{H}), 4.60(\mathrm{~s}, 1 \mathrm{H}), 4.37-4.34$ (m, 2H), 2.78-2.74 (m, 1H), $2.48(\mathrm{~m}, 1 \mathrm{H}), 2.34-2.27(\mathrm{~m}, 2 \mathrm{H}), 2.19-2.13(\mathrm{~m}, 2 \mathrm{H}), 1.92-1.86(\mathrm{~m}$, 2H), 1.80-1.77 (m, 1H), 1.72-1.66 (m, 4H), 1.60-1.39 (m, 3H), $0.92(\mathrm{~d}, J=6.9 \mathrm{~Hz}, 3 \mathrm{H}), 0.76(\mathrm{~d}$, $J=6.9 \mathrm{~Hz}, 3 \mathrm{H}) ;{ }^{13} \mathrm{C} \mathrm{NMR}\left(125 \mathrm{MHz}, \mathrm{CDCl}_{3}\right) \delta 143.4,132.9,132.8,122.5,79.4,77.0,76.7$, 49.7, 49.0, 39.0, 33.5, 33.1, 29.8, 28.6, 22.7, 22.7, 21.6, 15.7; IR (neat) 3356, 2956, 2924, 2845, $1676 \mathrm{~cm}^{-1}$; HRMS calcd for $\mathrm{C}_{18} \mathrm{H}_{26} \mathrm{O}_{2} \mathrm{Na}(\mathrm{M}+\mathrm{Na})^{+} 297.1831$, found 297.1845; $[\alpha]^{20}{ }_{\mathrm{D}}=-12.03(c$ $\left.0.69, \mathrm{CHCl}_{3}\right)$. 
Mitsunobu of 13: To a stirred solution of 13 ( $7 \mathrm{mg}, 0.025 \mathrm{mmol})$, triphenylphosphine (7 mg, $0.035 \mathrm{mmol})$ and benzoic acid (5 $\mathrm{mg}, 0.05 \mathrm{mmol})$ in $1 \mathrm{~mL}$ of THF at room temperature was slowly added a solution of DEAD $(6 \mathrm{mg}, 0.035 \mathrm{mmol})$ in $1 \mathrm{~mL}$ of THF. The reaction was stirred at room temperature for $10 \mathrm{~h}$ and $1 \mathrm{~mL}$ of a $1 \mathrm{M}$ solution of $\mathrm{NaOMe}$ in $\mathrm{MeOH}$ was added. The reaction was stirred for an additional $10 \mathrm{~h}$, portioned between $\mathrm{H}_{2} \mathrm{O}(3 \mathrm{~mL})$ and $\mathrm{Et}_{2} \mathrm{O}(6 \mathrm{~mL})$, and the aqueous layer was extracted with EtOAc $(2 \times 2 \mathrm{~mL})$. The organic extracts were combined, dried, concentrated, and purified by column chromatography $\left(\mathrm{SiO}_{2}, 4: 1\right.$ EtOAc/petroleum ether) to give $5 \mathrm{mg}(71 \%)$ of $\mathbf{1 2}$ as a clear oil.

Acetate 14: A flask containing $150 \mathrm{mg}(0.547 \mathrm{mmol})$ of 12 and $5 \mathrm{~mL}$ of pyridine was cooled to $0{ }^{\circ} \mathrm{C} .558 \mathrm{mg}(5.47 \mathrm{mmol})$ of $\mathrm{Ac}_{2} \mathrm{O}$ followed by $10 \mathrm{mg}(0.081 \mathrm{mmol})$ of DMAP were then added. This solution was allowed to warm to rt and stirred at that temperature for an additional $30 \mathrm{~min}$. $10 \mathrm{~mL}$ of EtOAc was added and the layers separated. The organic layer was washed with $1 \mathrm{M}$ $\mathrm{HCl}(3 \times 15 \mathrm{~mL}), \mathrm{H}_{2} \mathrm{O}(2 \times 20 \mathrm{~mL})$, and brine $(1 \times 25 \mathrm{~mL})$. The organic extracts were dried, filtered, and concentrated to give $174 \mathrm{mg}(100 \%)$ of $14 .{ }^{1} \mathrm{H} \mathrm{NMR}\left(500 \mathrm{MHz}, \mathrm{CDCl}_{3}\right) \delta 5.59$ (br, 1H), $5.52(\mathrm{br}, 1 \mathrm{H}), 4.37-4.35(\mathrm{~m}, 2 \mathrm{H}), 2.57-2.54(\mathrm{~d}, J=16.5 \mathrm{~Hz}, 1 \mathrm{H}), 2.43-2.25(\mathrm{~m}, 5 \mathrm{H}), 2.01$ (s, 3H), 1.92-1.88 (m, 2H), 1.82-1.71 (m, 5H), 1.62-1.56 (m, 1H), 1.45-1.43 (m, 1H), $0.94(\mathrm{~d}, J$ $=6.9 \mathrm{~Hz}, 3 \mathrm{H}), 0.78(\mathrm{~d}, J=6.8 \mathrm{~Hz}, 3 \mathrm{H}) ;{ }^{13} \mathrm{C} \mathrm{NMR}\left(125 \mathrm{MHz}, \mathrm{CDCl}_{3}\right) \delta 171.1,146.0,132.8$, $129.4,122.5,81.1,76.9,76.9,49.6,49.1,39.0,33.2,30.4,30.2,28.7,22.8,22.7,21.6,21.2$, 15.7; IR (neat) 2957, 2924, $1734 \mathrm{~cm}^{-1}$; HRMS calcd for $\mathrm{C}_{20} \mathrm{H}_{28} \mathrm{O}_{3}(\mathrm{M})^{+} 316.2038$, found $316.2036 ;[\alpha]^{20}=-68.83\left(c 0.58, \mathrm{CHCl}_{3}\right)$. 
Epoxide 15: $174 \mathrm{mg}(0.547 \mathrm{mmol})$ of 14 was diluted with $10 \mathrm{~mL}$ of $\mathrm{CH}_{2} \mathrm{Cl}_{2}$. This solution was cooled to $0{ }^{\circ} \mathrm{C}$ and $103 \mathrm{mg}(0.602 \mathrm{mmol}, 100 \%$ pure $)$ of $m$-CPBA was added in a single portion. The solution was kept at $0{ }^{\circ} \mathrm{C}$ until no starting material remained by TLC, typically $1.5 \mathrm{~h}$. The solution was then cooled to $-78{ }^{\circ} \mathrm{C}$, where $\mathrm{O}_{3}$ was bubbled through the solution until a faint blue color persisted. This solution was purged with $\mathrm{N}_{2}$ to remove excess ozone at $-78{ }^{\circ} \mathrm{C}$, then 100 $\mu \mathrm{L}$ of $\mathrm{SMe}_{2}$ was added. The colorless solution was allowed to warm to rt, and it was stirred for an additional $2 \mathrm{~h}$. The solution was washed with a $10 \%$ aqueous $\mathrm{Na}_{2} \mathrm{CO}_{3}$ solution, dried, filtered, and concentrated to give the crude dione. Purification by column chromatography $\left(\mathrm{SiO}_{2}, 5: 1\right.$ petroleum ether/ EtOAc) provided $100 \mathrm{mg}$ of $\mathbf{1 5}$ as a white crystalline solid (as well as $61 \mathrm{mg}$ (31\%) of the diepoxide 18 (see X-ray data). mp $154-156{ }^{\circ} \mathrm{C} ;{ }^{1} \mathrm{H}$ NMR $\left(500 \mathrm{MHz}, \mathrm{CDCl}_{3}\right) \delta 5.13$ $(\mathrm{dd}, J=2.2,9.7 \mathrm{~Hz}, 1 \mathrm{H}), 4.58(\mathrm{~m}, 1 \mathrm{H}), 4.15(\mathrm{~s}, 1 \mathrm{H}), 3.83-3.80(\mathrm{dd}, J=5.5,12.5 \mathrm{~Hz}, 1 \mathrm{H}), 3.11$ (s, 1H), 2.99-2.94 (m, 1H), 2.45-2.18 (m, 6H), 2.04-2.00 (m, 4H), 1.75-1.71 (m, 1H), 1.49-1.42 $(\mathrm{m}, 2 \mathrm{H}), 1.35(\mathrm{~s}, 3 \mathrm{H}), 0.88(\mathrm{~d}, J=6.9 \mathrm{~Hz}, 3 \mathrm{H}), 0.70(\mathrm{~d}, J=6.8 \mathrm{~Hz}, 3 \mathrm{H}) ;{ }^{13} \mathrm{C} \mathrm{NMR}(125 \mathrm{MHz}$, $\left.\mathrm{CDCl}_{3}\right) \delta 211.2,208.9,170.5,86.2,80.6,78.6,61.6,55.6,47.0,41.5,40.5,36.0,31.9,27.4,27.3$, 23.8, 22.9, 21.0, 20.3, 15.0; IR (neat) 2959, 2929, 2854, 1737, $1718 \mathrm{~cm}^{-1}$; HRMS calcd for

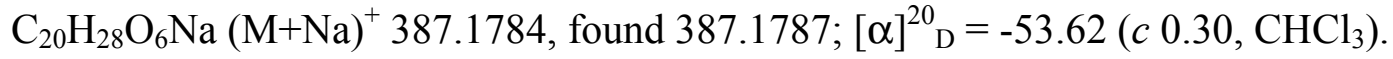

Dione 16: A flame dried round-bottomed flask was charged with $396 \mathrm{mg}(1.0 \mathrm{mmol})$ of $\mathrm{WCl}_{6}$ followed $10 \mathrm{~mL}$ of dry THF. The black suspension was cooled to $-78{ }^{\circ} \mathrm{C}$ and $1.25 \mathrm{~mL}(1.6 \mathrm{M}$ in hexane, $2 \mathrm{mmol}$ ) of $n-\mathrm{BuLi}$ was added dropwise. The mixture was then allowed to warm to rt during which time it underwent a number of color changes. $7 \mathrm{~mL}$ of this solution was then added to a solution of $\mathbf{1 5}(99 \mathrm{mg}, 0.272 \mathrm{mmol})$ in $2 \mathrm{~mL}$ of THF at $0{ }^{\circ} \mathrm{C}$. The reaction was 
stirred for 5 min at $0{ }^{\circ} \mathrm{C}$ then warmed to rt. After stirring for $30 \mathrm{~min}, 10 \mathrm{~mL}$ of saturated aqueous $\mathrm{NaHCO}_{3}$ was slowly added. The biphasic solution was extracted with $\mathrm{CH}_{2} \mathrm{Cl}_{2}(4 \times 15 \mathrm{~mL})$, dried with $\mathrm{MgSO}_{4}$, filtered, and concentrated to give the crude dione. Purification using column chromatography $\left(\mathrm{SiO}_{2}, 4: 1\right.$ petroleum ether/ EtOAc) yielded $88 \mathrm{mg}(93 \%)$ of $\mathbf{1 6}$ as a white crystalline solid. mp $156-157{ }^{\circ} \mathrm{C} ;{ }^{1} \mathrm{H}$ NMR $\left(500 \mathrm{MHz}, \mathrm{CDCl}_{3}\right) \delta 5.48(\mathrm{~s}, 1 \mathrm{H}), 5.15(\mathrm{~d}, J=8.5$ $\mathrm{Hz}, 1 \mathrm{H}), 4.26(\mathrm{~s}, 1 \mathrm{H}), 4.18(\mathrm{~m}, 1 \mathrm{H}), 3.72-3.69(\mathrm{dd}, J=5.5,12.5 \mathrm{~Hz}, 1 \mathrm{H}), 3.02-2.96(\mathrm{~m}, 1 \mathrm{H})$, 2.66-2.62 (m, 1H), 2.53-2.47 (m, 2H), 2.34-2.30 (m, 2H), 2.26-2.18 (m, 1H), 2.04 (s, 3H), 1.96-1.92 (m, 1H), 1.84-1.76 (m, 2H), 1.67 (s, 3H), 1.48-1.42 (m, 1H), $0.92(\mathrm{~d}, J=6.9 \mathrm{~Hz}, 3 \mathrm{H})$, $0.75(\mathrm{~d}, J=6.8 \mathrm{~Hz}, 3 \mathrm{H}) ;{ }^{13} \mathrm{C} \mathrm{NMR}\left(125 \mathrm{MHz}, \mathrm{CDCl}_{3}\right) \delta 211.4,208.1,170.6,130.7,123.3,86.3$, 83.1, 78.9, 47.3, 44.2, 41.6, 36.4, 36.2, 27.7, 27.6, 23.3, 22.8, 21.3, 20.3, 15.5; IR (neat) 2956, 1733, $1715 \mathrm{~cm}^{-1}$; HRMS calcd for $\mathrm{C}_{20} \mathrm{H}_{28} \mathrm{O}_{5} \mathrm{Na}(\mathrm{M}+\mathrm{Na})^{+} 371.1834$, found $371.1822 ;[\alpha]^{20}{ }_{\mathrm{D}}=-$ $56.39\left(\right.$ c $\left.0.31, \mathrm{CHCl}_{3}\right)$.

Allylic acetate 17: A $10 \mathrm{~mL}$ round-bottomed flask was charged with $10 \mathrm{mg}(0.028 \mathrm{mmol})$ of $\mathbf{1 8}$ along with $2 \mathrm{~mL}$ of THF. The solution was cooled to $-78{ }^{\circ} \mathrm{C}$ where $9.8 \mathrm{mg}$ of TBSOTf $(0.037$ mmol) was added. KHMDS ( $0.5 \mathrm{M}$ in toluene, $112 \mu \mathrm{L}, 0.056 \mathrm{mmol})$ was slowly added to the cooled solution. After $5 \mathrm{~min}$, the reaction was quenched with $2 \mathrm{~mL}$ of saturated aqueous $\mathrm{NaHCO}_{3}$, extracted with $\mathrm{CH}_{2} \mathrm{Cl}_{2}(4 \times 3 \mathrm{~mL})$, dried, filtered, and concentrated to give the silyl enol ether. To this TBS enol ether was added $1 \mathrm{~mL}$ of dry THF. This solution was cooled to 0 ${ }^{\circ} \mathrm{C}$ and $1 \mathrm{~mL}$ of a premade ylide (made from $86 \mathrm{mg} \mathrm{Ph}{ }_{3} \mathrm{PCH}_{2} \mathrm{Br}$ and $27 \mathrm{mg}$ of $t-\mathrm{BuOK}$ in $2.5 \mathrm{~mL}$ of THF) solution was added. The solution was stirred for $5 \mathrm{~min}$ at $0{ }^{\circ} \mathrm{C}$ then warmed to rt. After stirring for $1 \mathrm{~h}, 1.5 \mathrm{~mL}$ of $1 \mathrm{M} \mathrm{HCl}$ was added, and the solution was stirred overnight at rt. $5 \mathrm{~mL}$ of $\mathrm{CH}_{2} \mathrm{Cl}_{2}$ was then added, the layers were separated, and the aqueous layer further extracted 
with $\mathrm{CH}_{2} \mathrm{Cl}_{2}(3 \times 5 \mathrm{~mL})$. The organic extracts were dried, filtered, concentrated, and purified by column chromatography $\left(\mathrm{SiO}_{2}, 4: 1\right.$ hexanes/ EtOAc) to give $6.0 \mathrm{mg}$ (61\% over two steps) of 17 as a pale yellow oil. ${ }^{1} \mathrm{H} \mathrm{NMR}\left(500 \mathrm{MHz}, \mathrm{CDCl}_{3}\right) \delta 5.49(\mathrm{br}, 1 \mathrm{H}), 5.46(\mathrm{~s}, 1 \mathrm{H}), 5.08-5.04(\mathrm{~m}$, 2H), $4.21(\mathrm{~s}, 1 \mathrm{H}), 4.09-4.06(\mathrm{dd}, J=5.3,8.8 \mathrm{~Hz}, 1 \mathrm{H}), 3.10-3.07(\mathrm{dd}, J=5.4,13.9 \mathrm{~Hz}, 1 \mathrm{H}), 3.01$ $(\mathrm{t}, J=12.9 \mathrm{~Hz}, 1 \mathrm{H}), 2.66-2.62(\mathrm{dd}, J=6.8,12.3 \mathrm{~Hz}, 1 \mathrm{H}), 2.45(\mathrm{t}, J=7.9 \mathrm{~Hz}, 1 \mathrm{H}), 2.40(\mathrm{~d}, J=$ $13.9 \mathrm{~Hz}, 1 \mathrm{H}), 2.25-2.15(\mathrm{~m}, 3 \mathrm{H}), 1.98(\mathrm{~s}, 3 \mathrm{H}), 1.87-1.74(\mathrm{~m}, 2 \mathrm{H}), 1.65(\mathrm{~s}, 3 \mathrm{H}), 1.50-1.47$ (m, $1 \mathrm{H}), 0.92-0.88(\mathrm{~d}, J=6.9 \mathrm{~Hz}, 3 \mathrm{H}), 0.86-0.77(\mathrm{~m}, 1 \mathrm{H}), 0.73(\mathrm{~d}, J=6.9 \mathrm{~Hz}, 3 \mathrm{H}) ;{ }^{13} \mathrm{C} \mathrm{NMR}(125$ $\left.\mathrm{MHz}, \mathrm{CDCl}_{3}\right) \delta 212.0,170.3,145.7,131.0,123.3,121.0,86.2,84.2,77.9,43.4,41.5,40.7,36.5$, $36.4,32.9,27.5,23.3,23.3,21.3,21.3,15.3$; IR (neat) $2957,1732,1716,1641 \mathrm{~cm}^{-1}$; HRMS calcd for $\mathrm{C}_{21} \mathrm{H}_{30} \mathrm{O}_{4}(\mathrm{M})^{+} 346.2144$, found 346.2127; $[\alpha]^{20}{ }_{\mathrm{D}}=-51.80\left(c 0.34, \mathrm{CHCl}_{3}\right)$.

Deacetoxyalcyonin Acetate 2: $120 \mathrm{mg}(0.20 \mathrm{mmol})$ of anhydrous $\mathrm{Yb}(\mathrm{OTf})_{3}$ was dried under reduced pressure $(0.1 \mathrm{mmHg})$ at $180{ }^{\circ} \mathrm{C}$ for $12 \mathrm{~h}$. The flask was cooled to $\mathrm{rt}$ and $2 \mathrm{~mL}$ of anhydrous THF was then added and the resulting opaque solution stirred at $\mathrm{rt}$ for $15 \mathrm{~min}$. The $\mathrm{Yb}(\mathrm{OTf})_{3}$ solution was cooled to $-78{ }^{\circ} \mathrm{C}$ and $125 \mu \mathrm{L}$ of $\mathrm{MeLi}\left(1.6 \mathrm{M}\right.$ in $\left.\mathrm{Et}_{2} \mathrm{O}, 0.2 \mathrm{mmol}\right)$ was added. The resulting red solution was stirred at $-78^{\circ} \mathrm{C}$ for $30 \mathrm{~min}$. In a separate flask, $6.6 \mathrm{mg}$ (0.02 mmol) of 17 was combined with $1.0 \mathrm{~mL}$ of anhydrous THF. This flask was then cooled to $-78{ }^{\circ} \mathrm{C}$. A $1 \mathrm{~mL}$ aliquot of the ytterbium solution was then rapidly added to the solution of $\mathbf{1 7}$. The solution was allowed to stir at $-78{ }^{\circ} \mathrm{C}$ for 30 min then quenched with saturated aqueous $\mathrm{NaHCO}_{3}$. The organics were extracted with $\mathrm{CH}_{2} \mathrm{Cl}_{2}(3 \times 4 \mathrm{~mL})$, dried, and concentrated. Purification of the residue via column chromatography $\left(\mathrm{SiO}_{2}, 10: 1\right.$ hexane/EtOAc) provided 3.5 $\mathrm{mg}$ of $2(51 \%, 66 \% \mathrm{BRSM})$ as well as $1.5 \mathrm{mg}$ of 17 . The spectral data for the synthetic deacetoxyalcyonin acetate 2 was identical to that reported for the natural product. ${ }^{3}$ The $[\alpha]^{20}{ }_{D}$ 
was found to be $-47.6\left(c 0.145, \mathrm{CHCl}_{3}\right)$, which compares favorably to the rotation reported by Overman. ${ }^{4}$ Recrystallization of the residue from hexanes (slow evaporation overnight) provided a crystal suitable for X-ray analysis. ${ }^{4}$ 

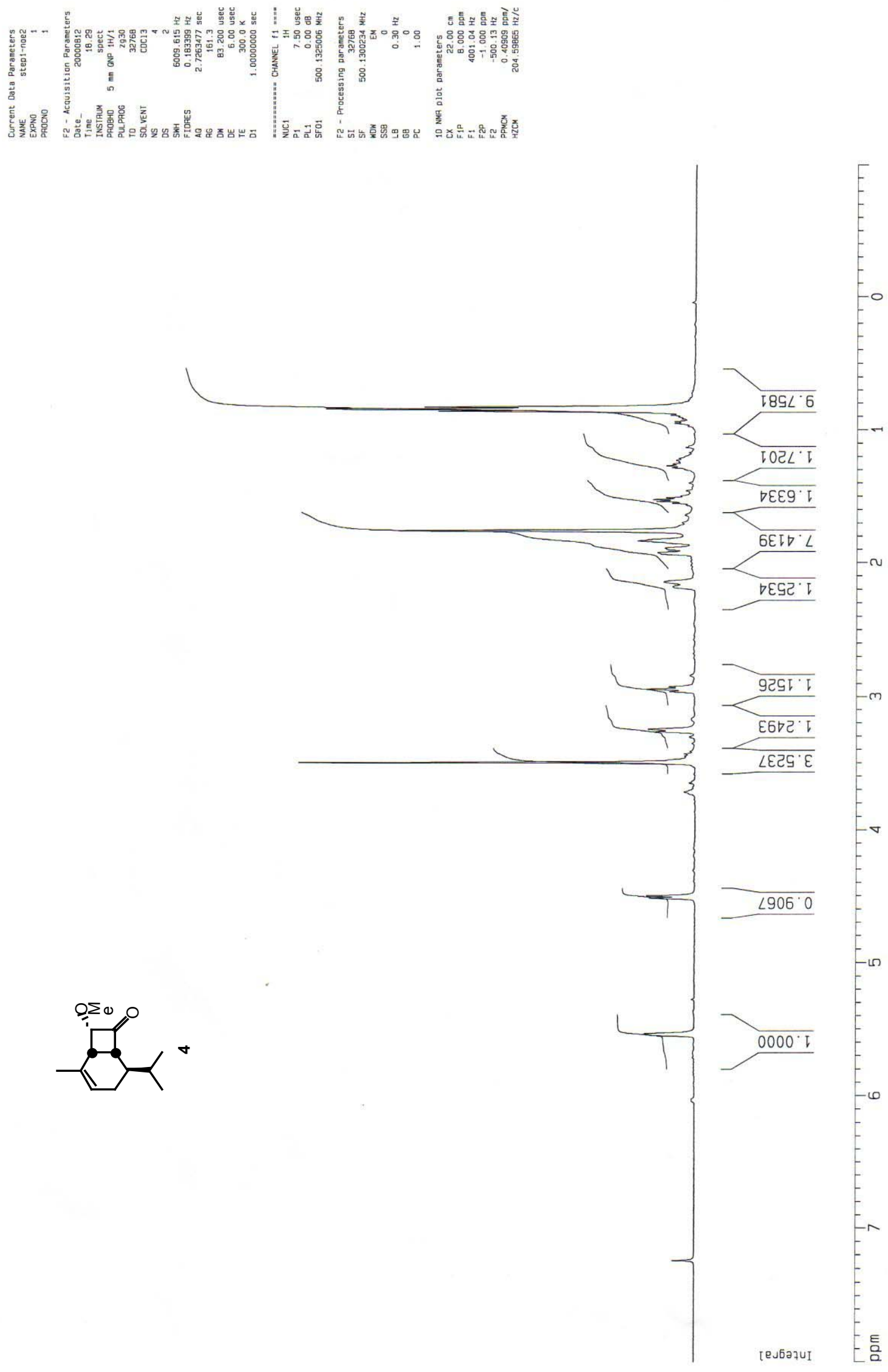

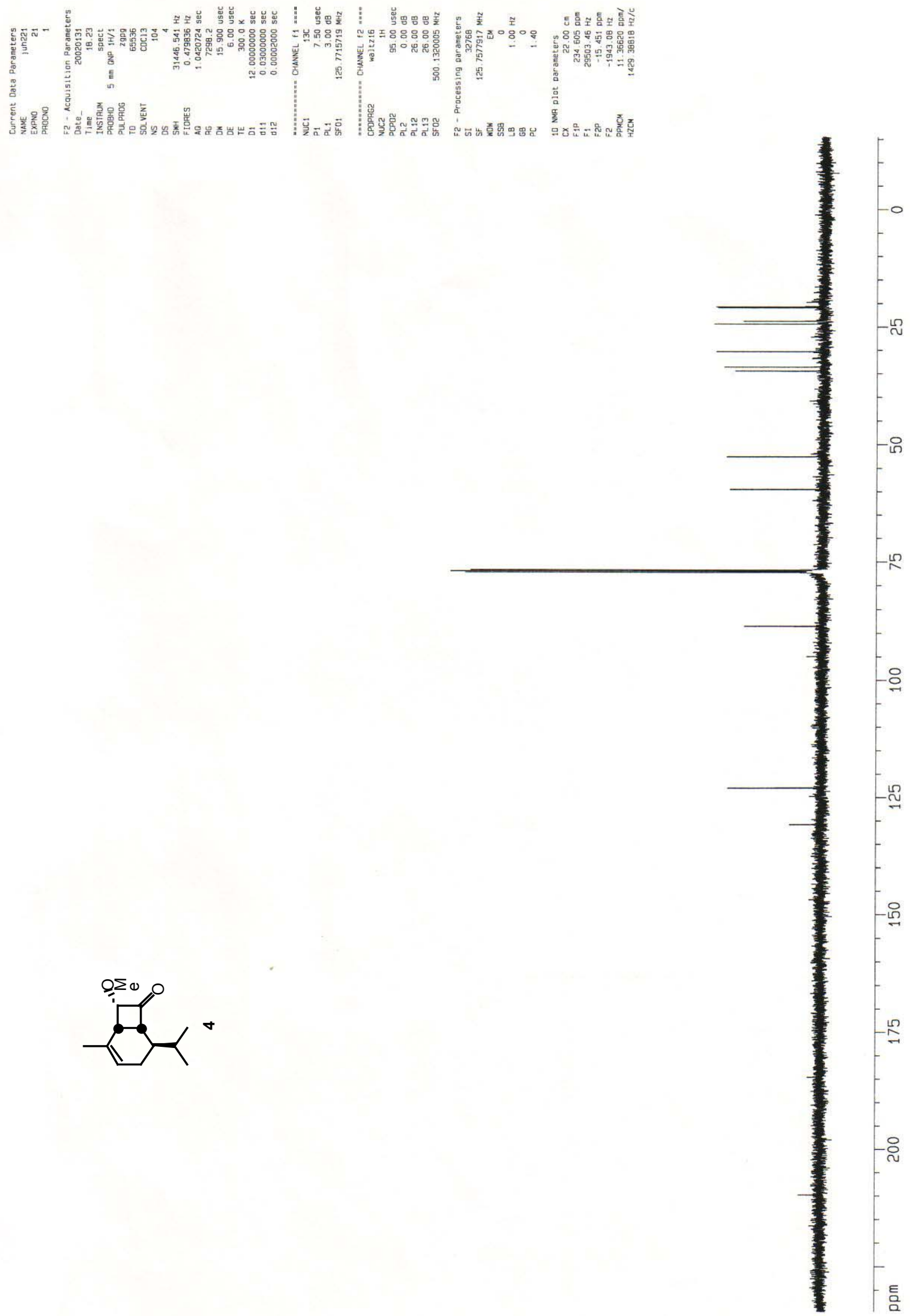

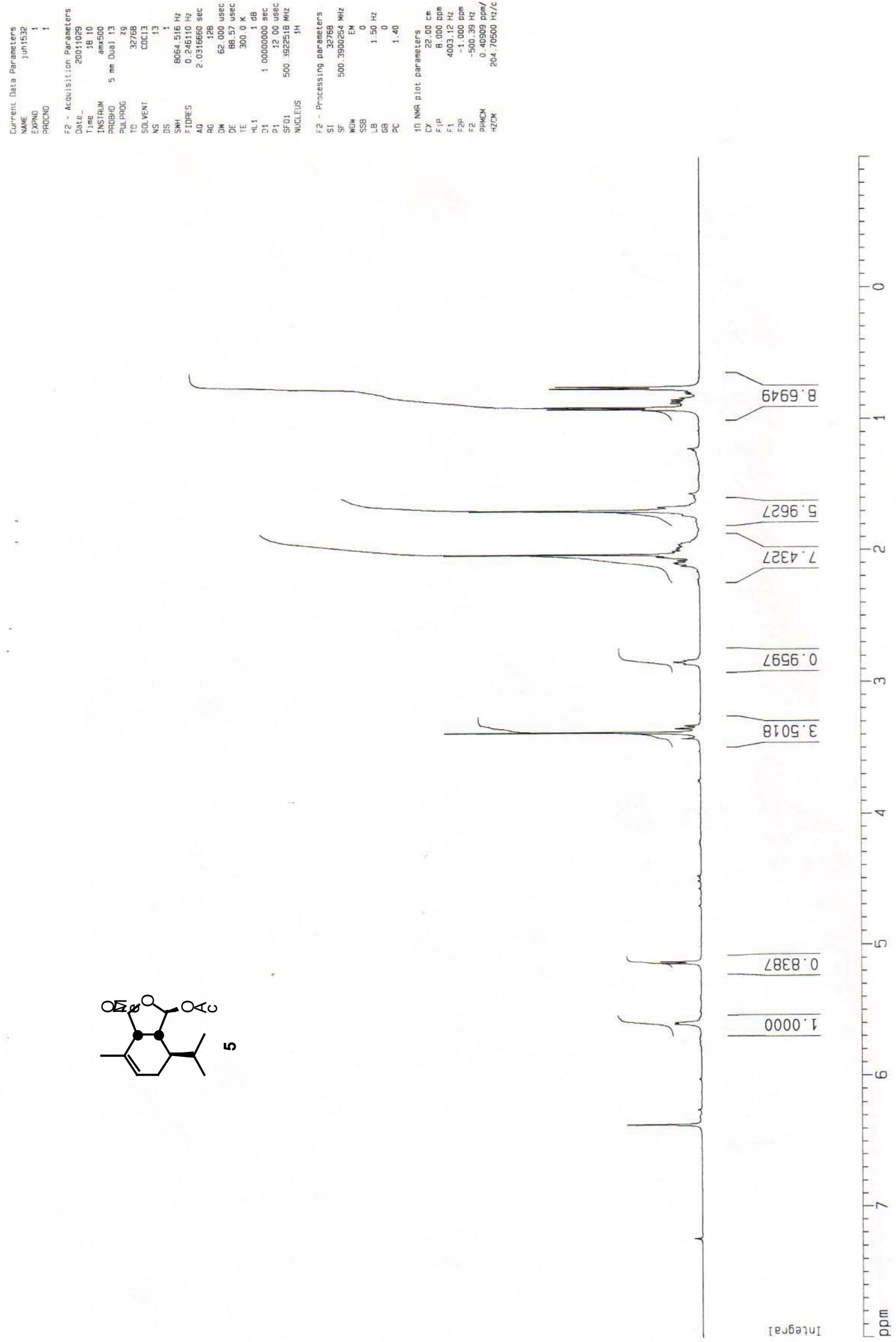

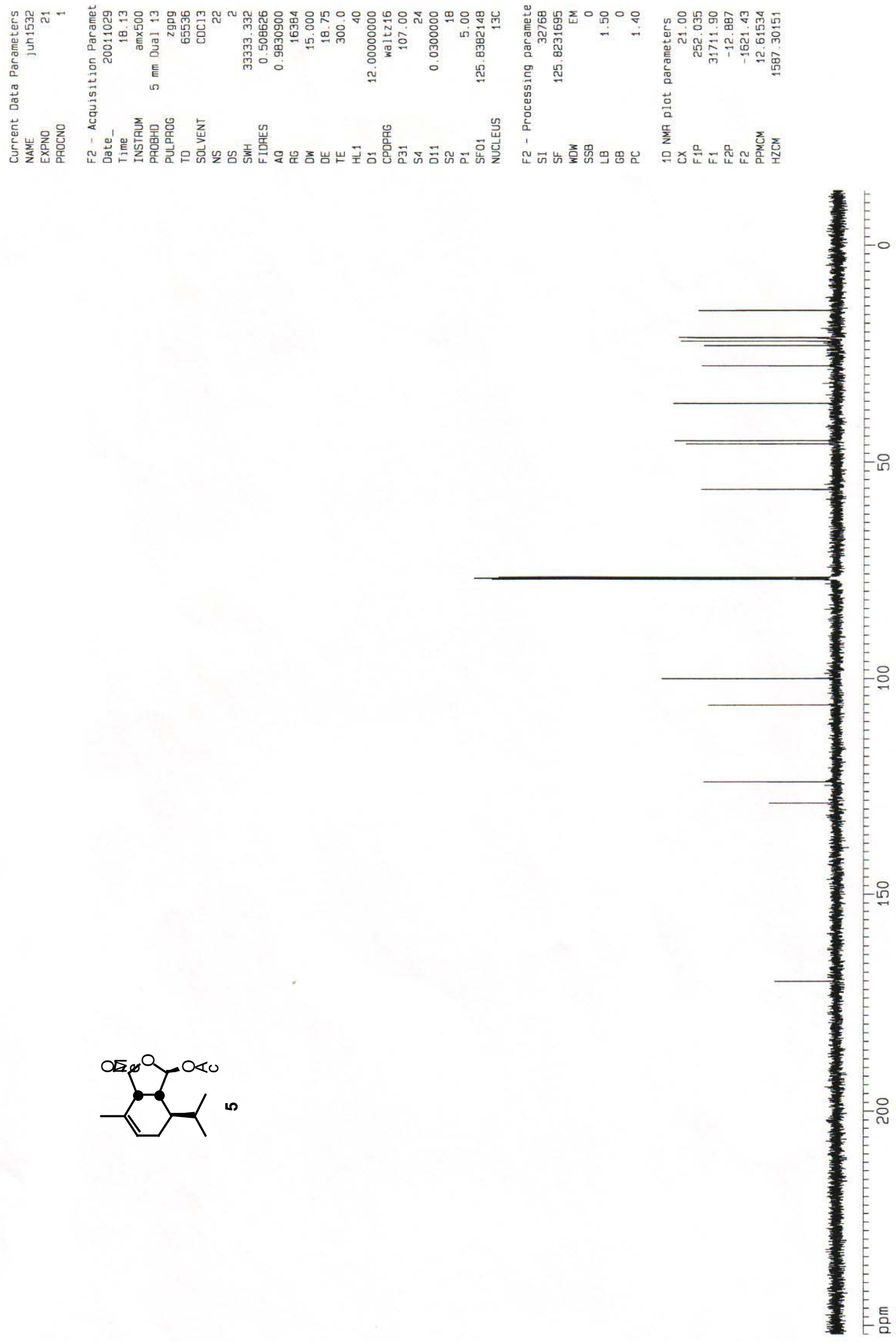

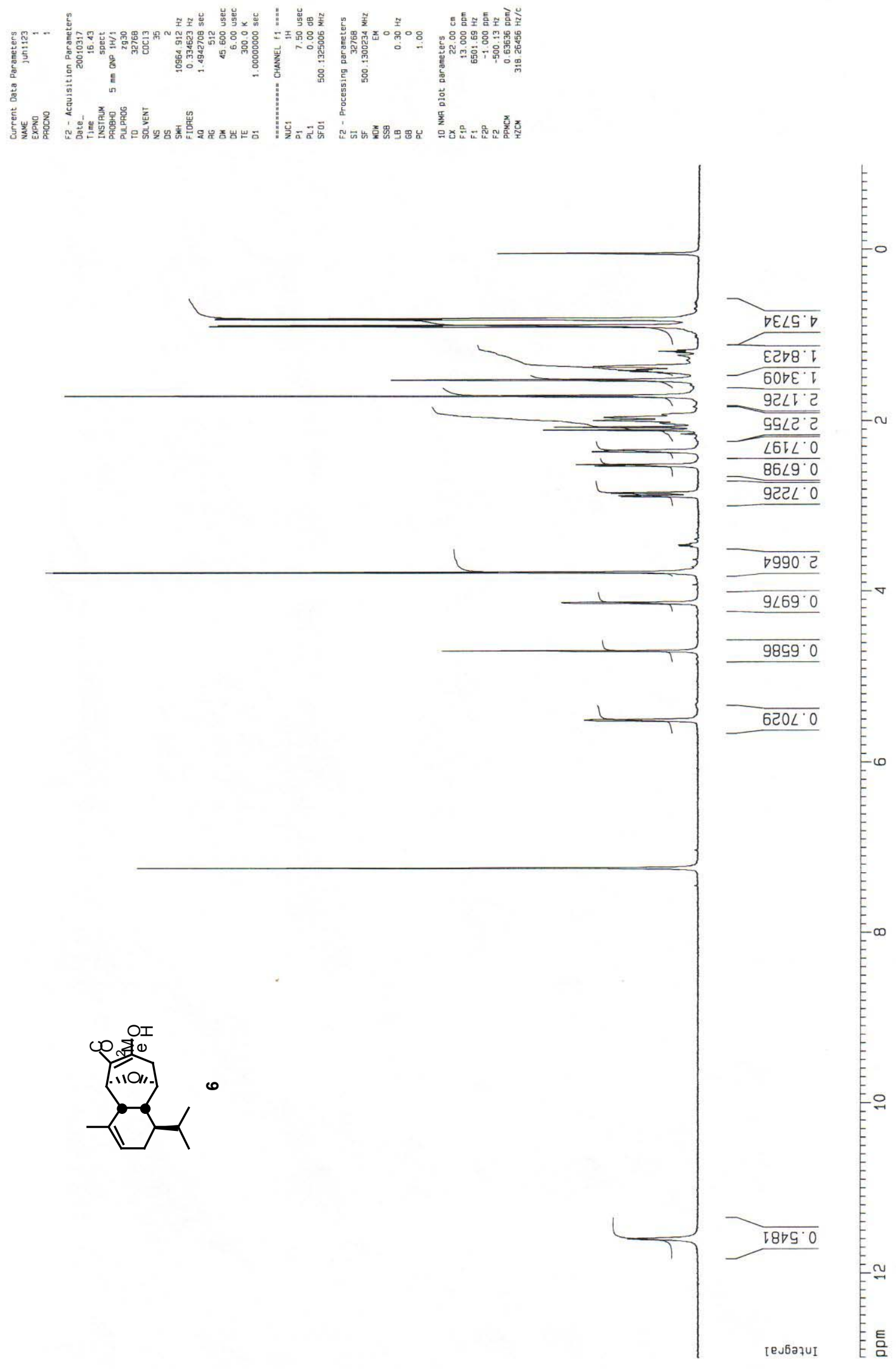

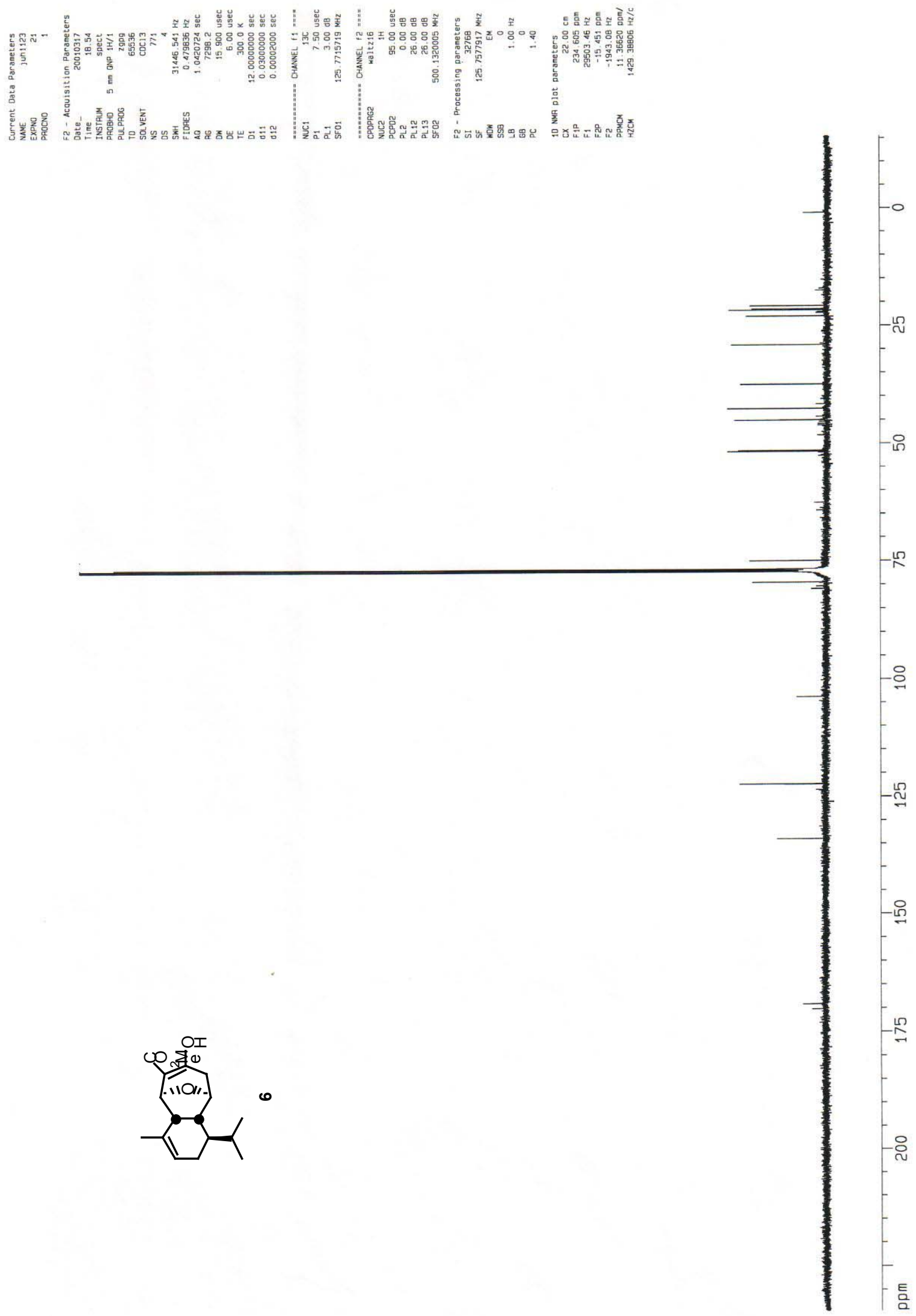

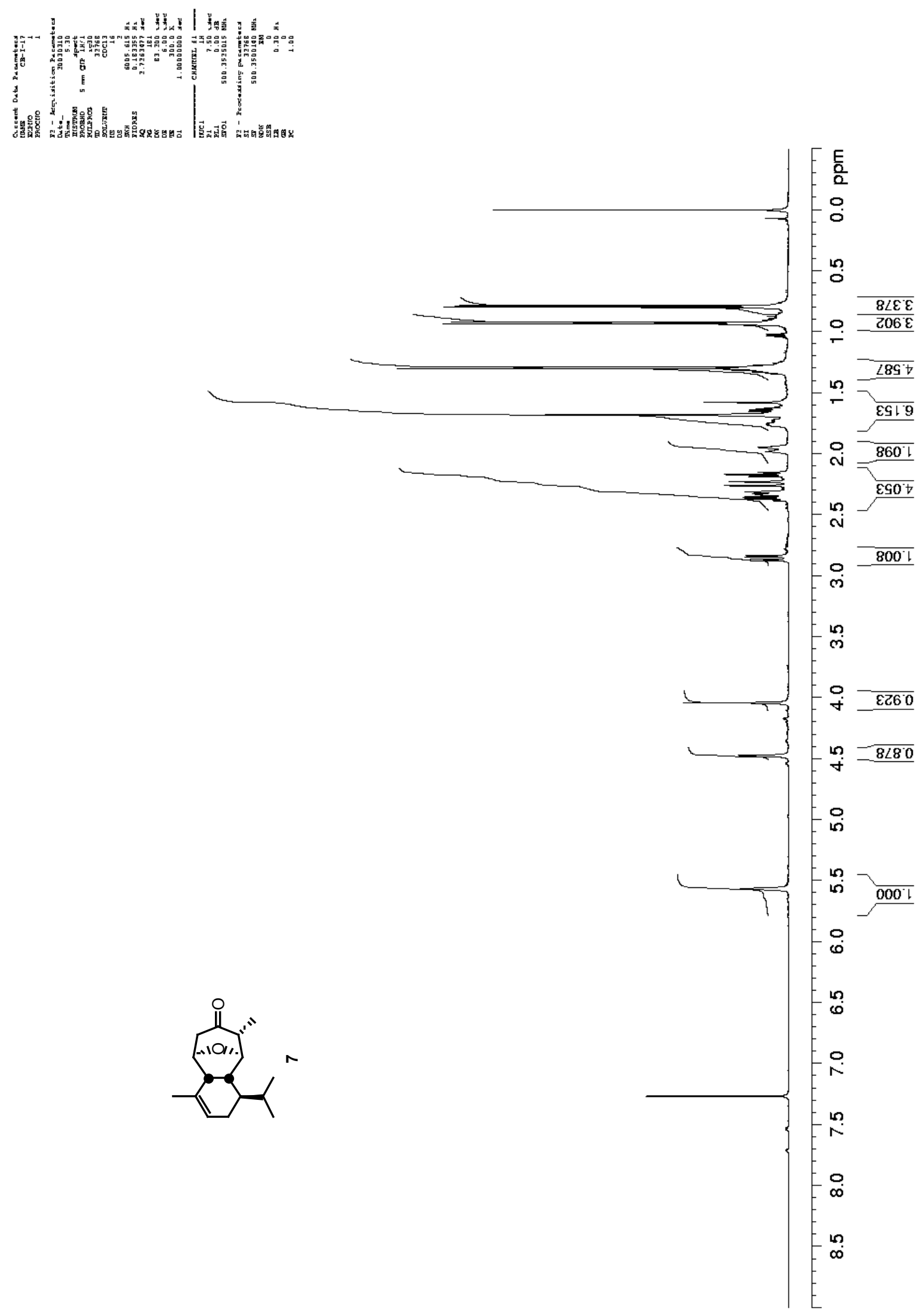


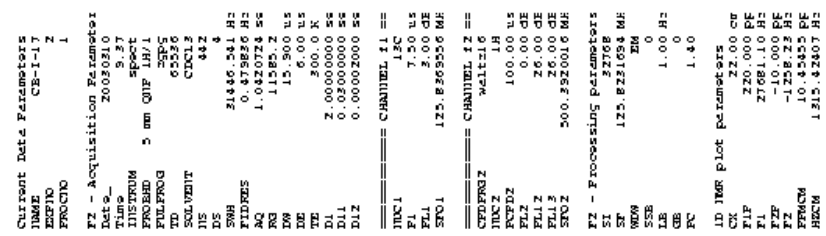

$$
\text { thent }
$$
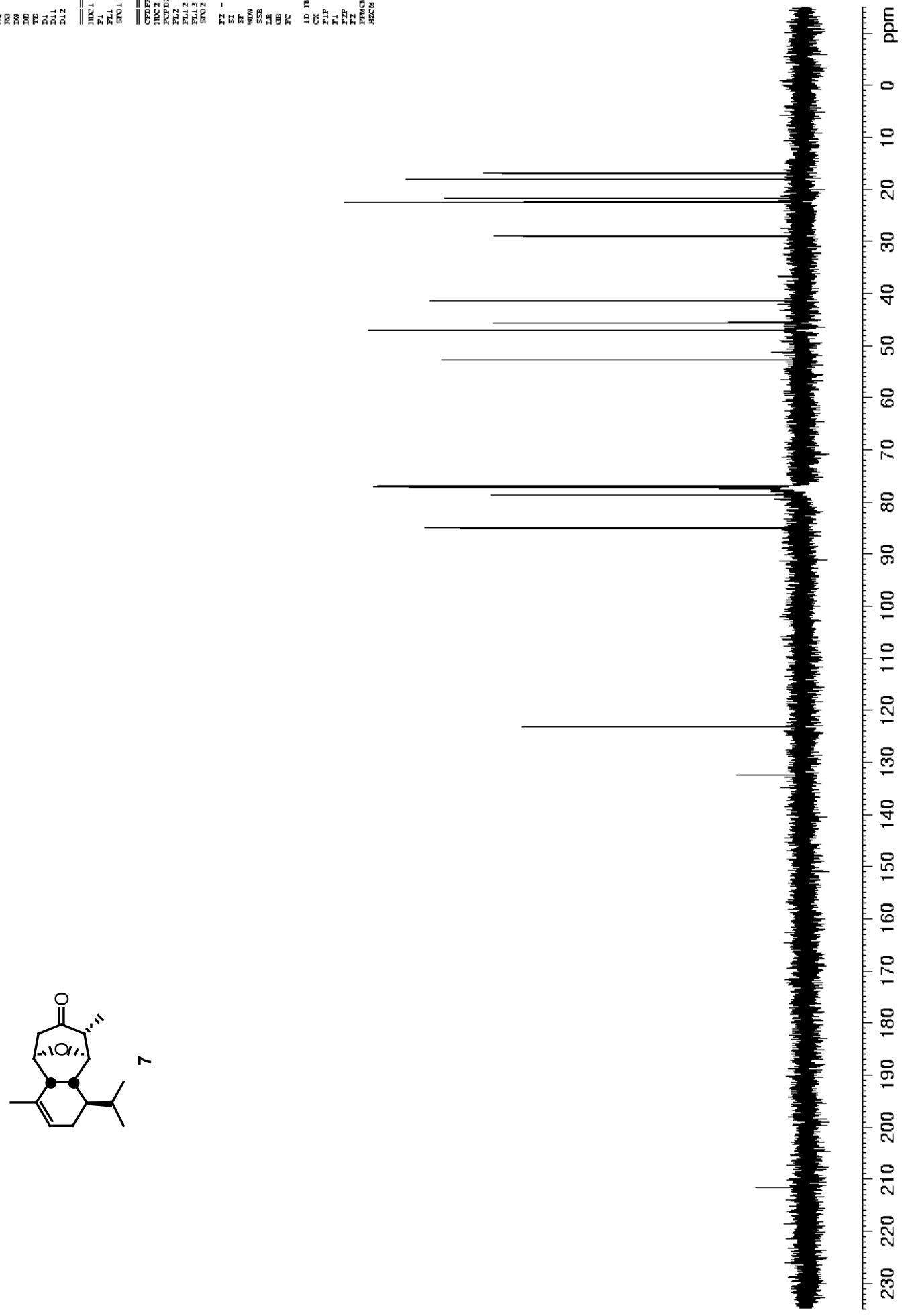

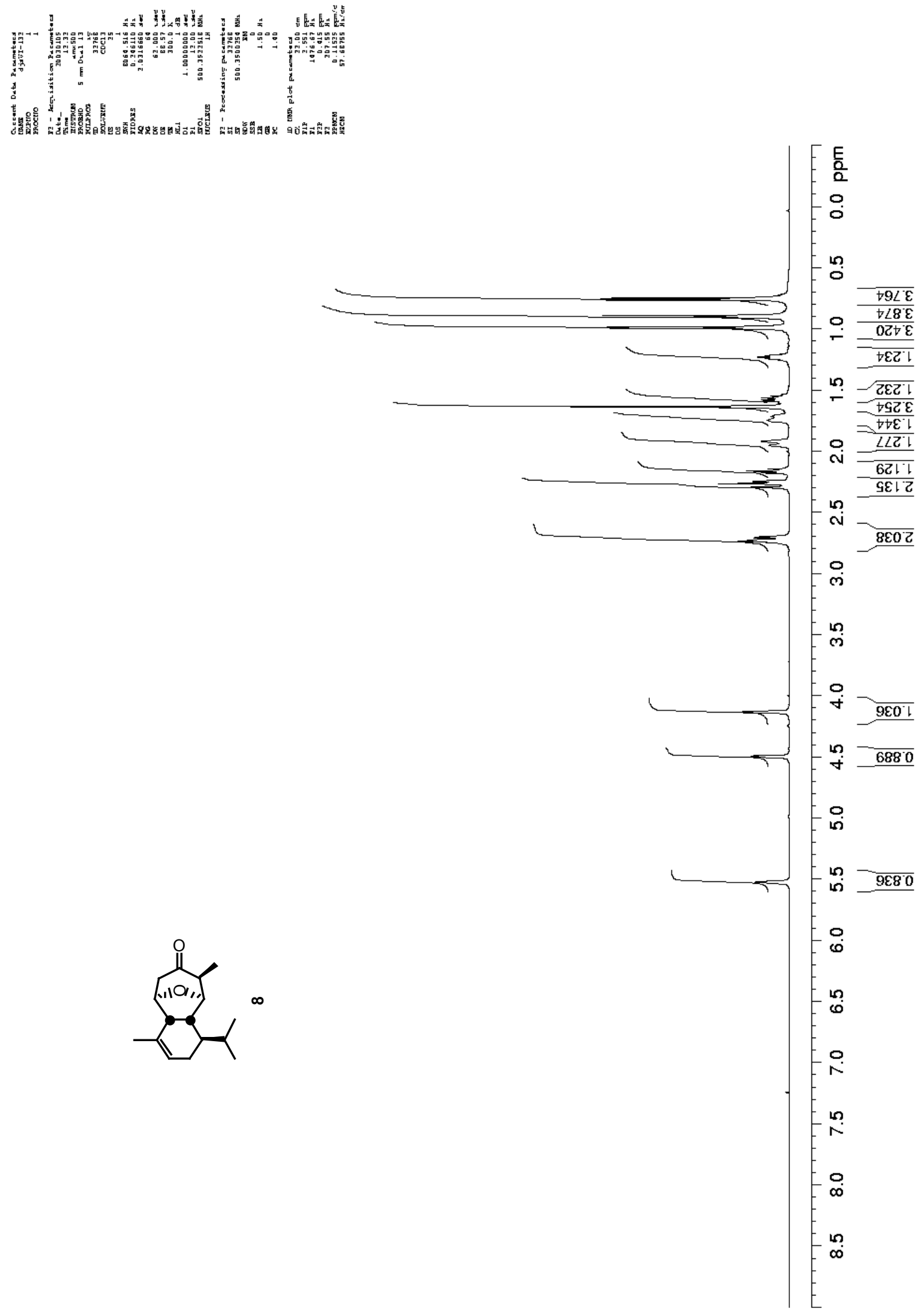

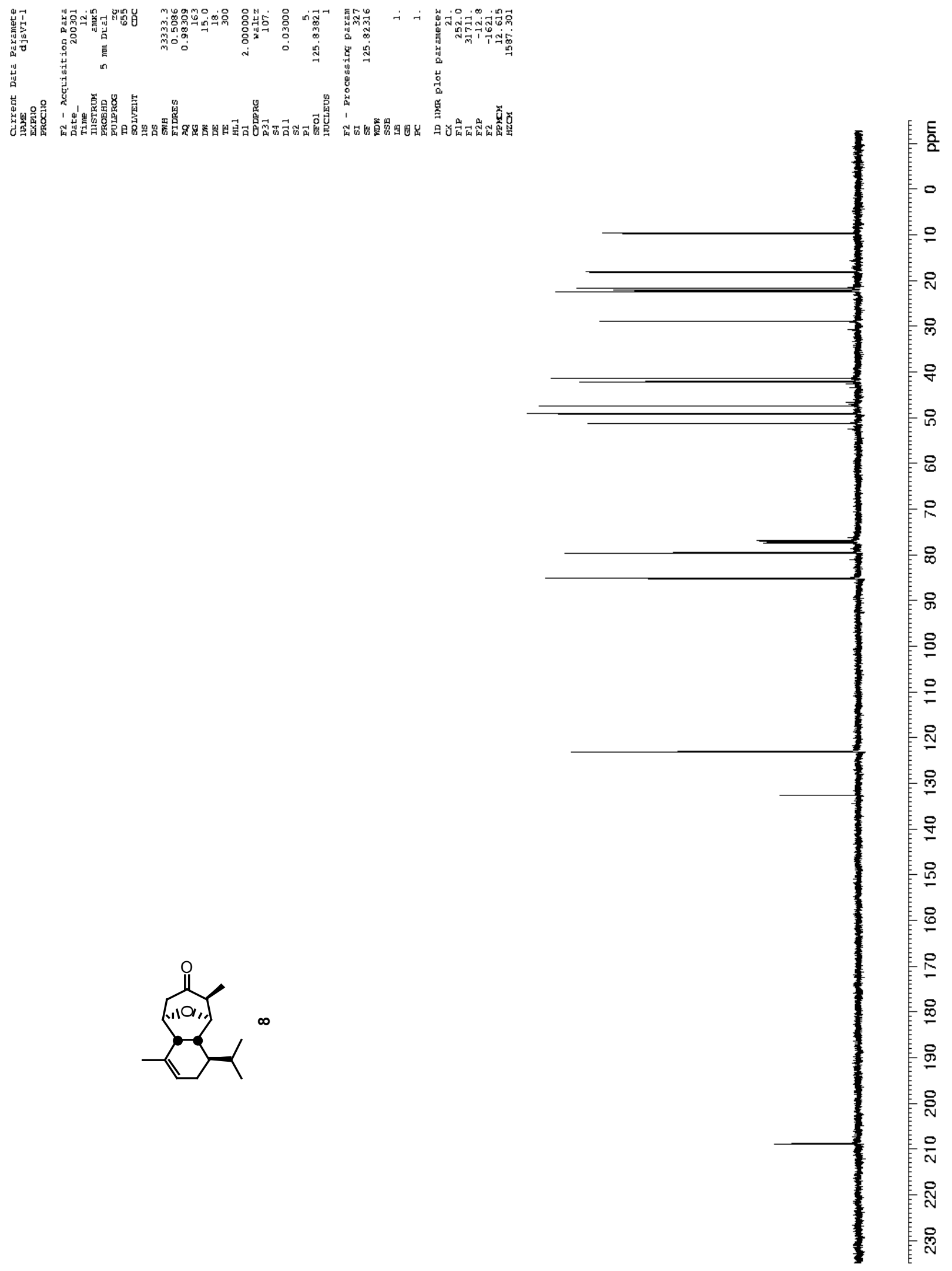

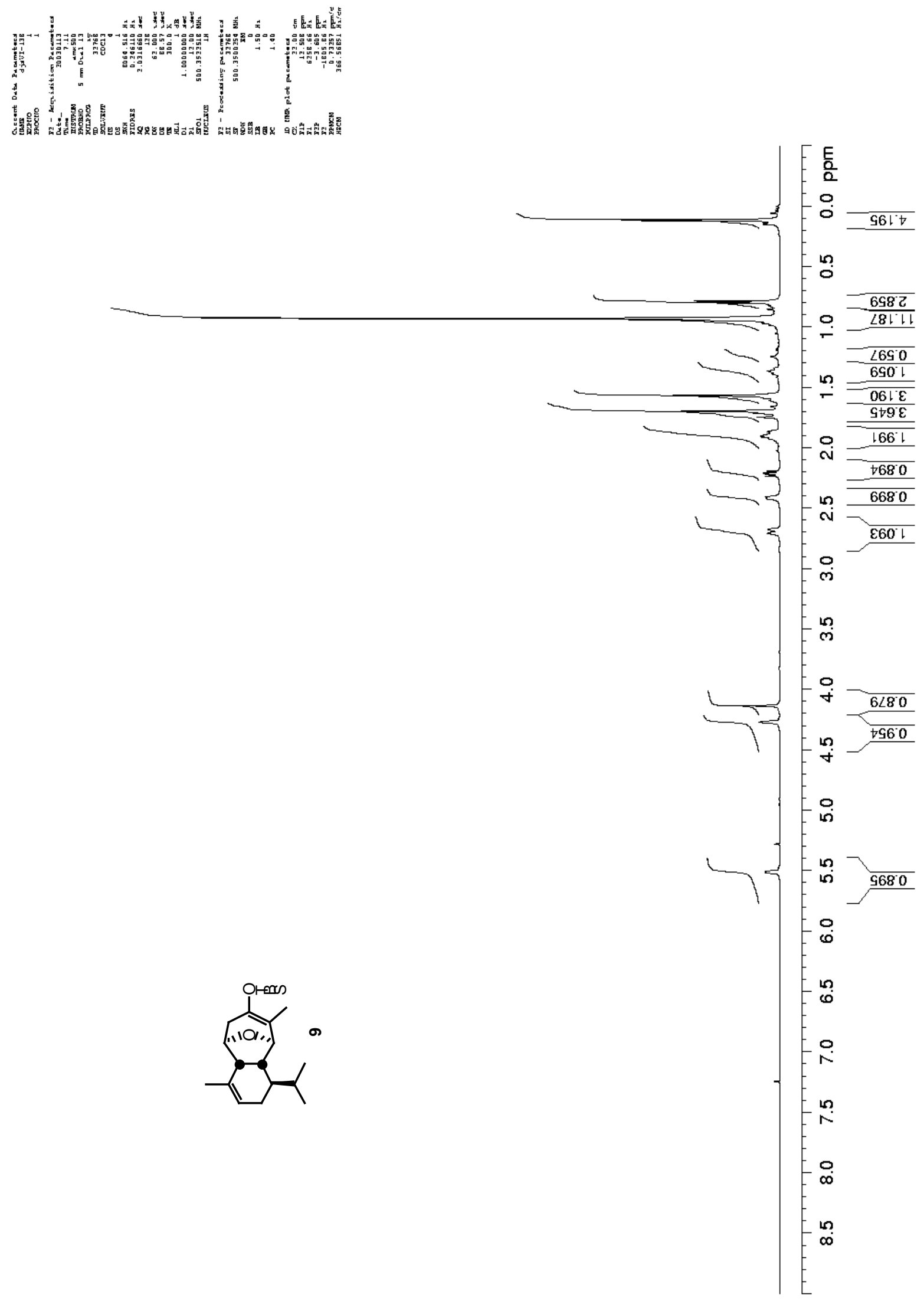

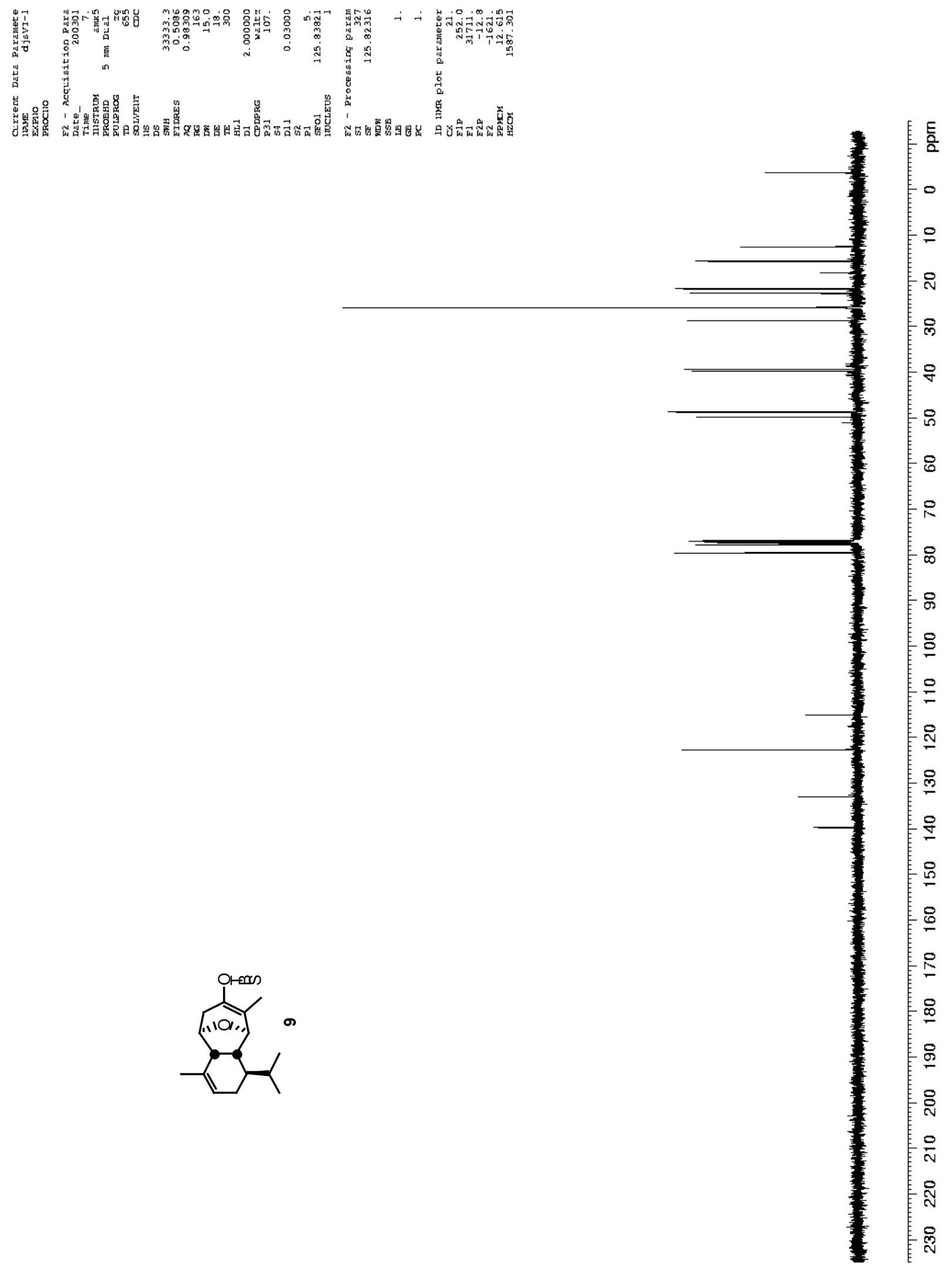

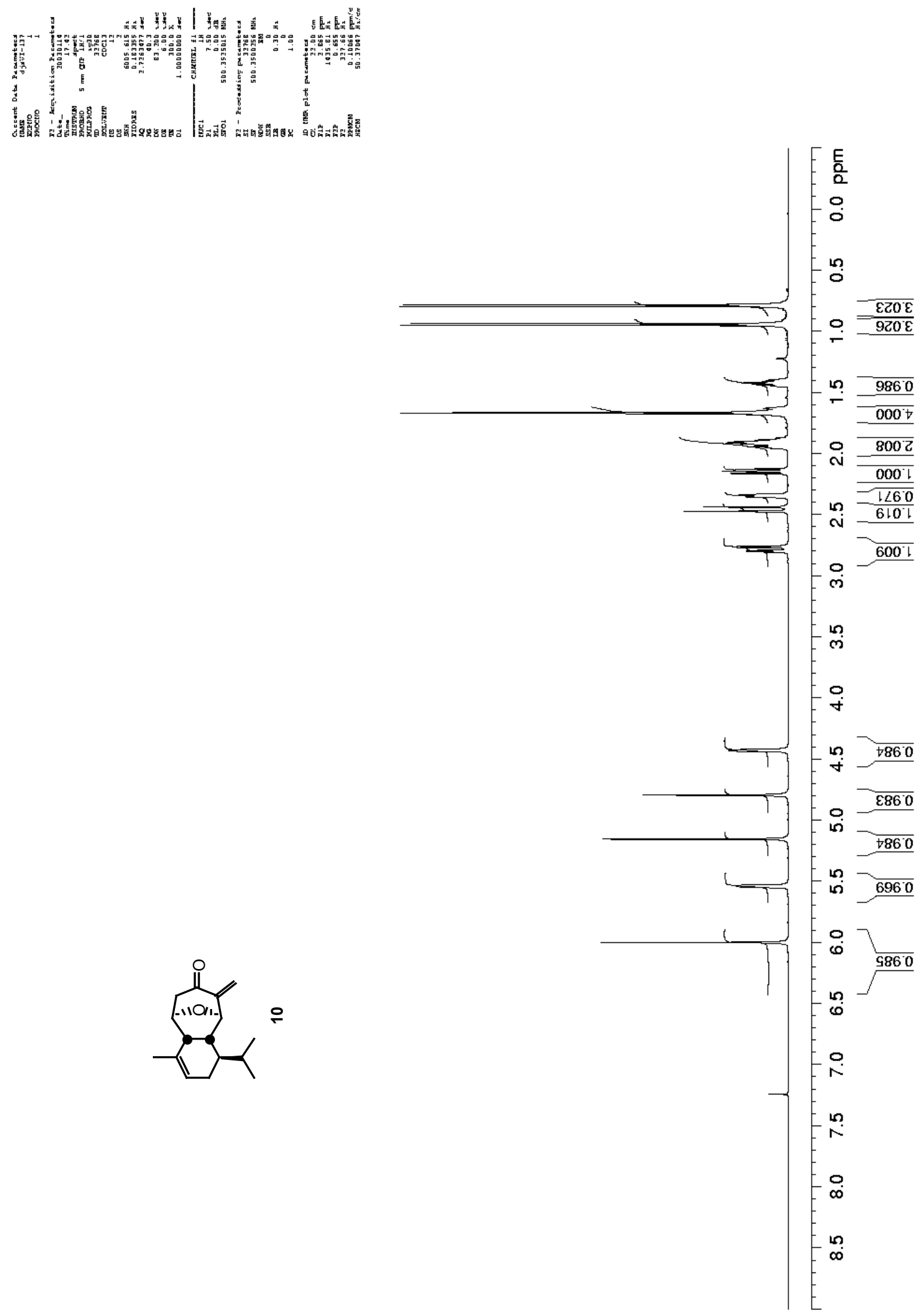

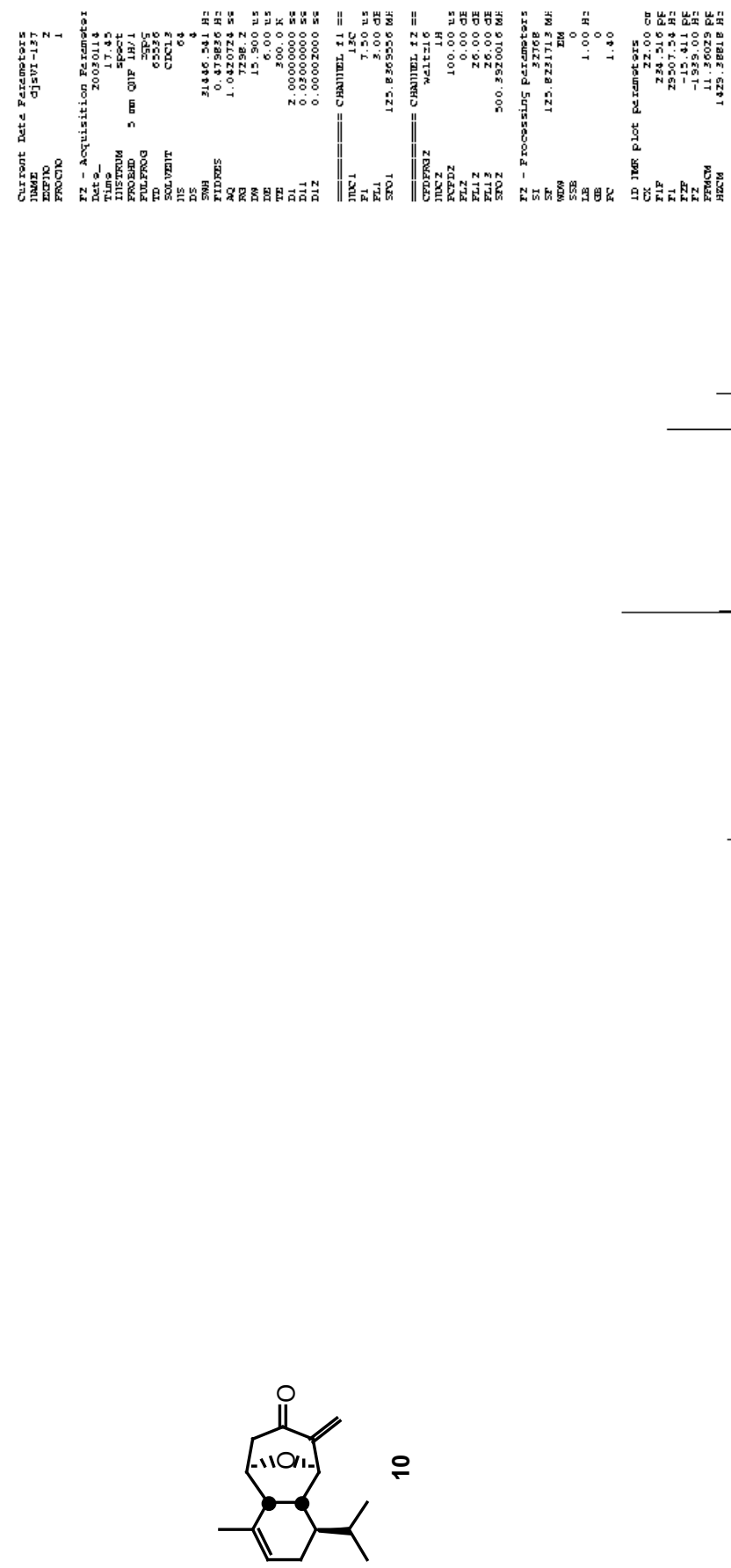

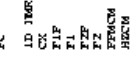

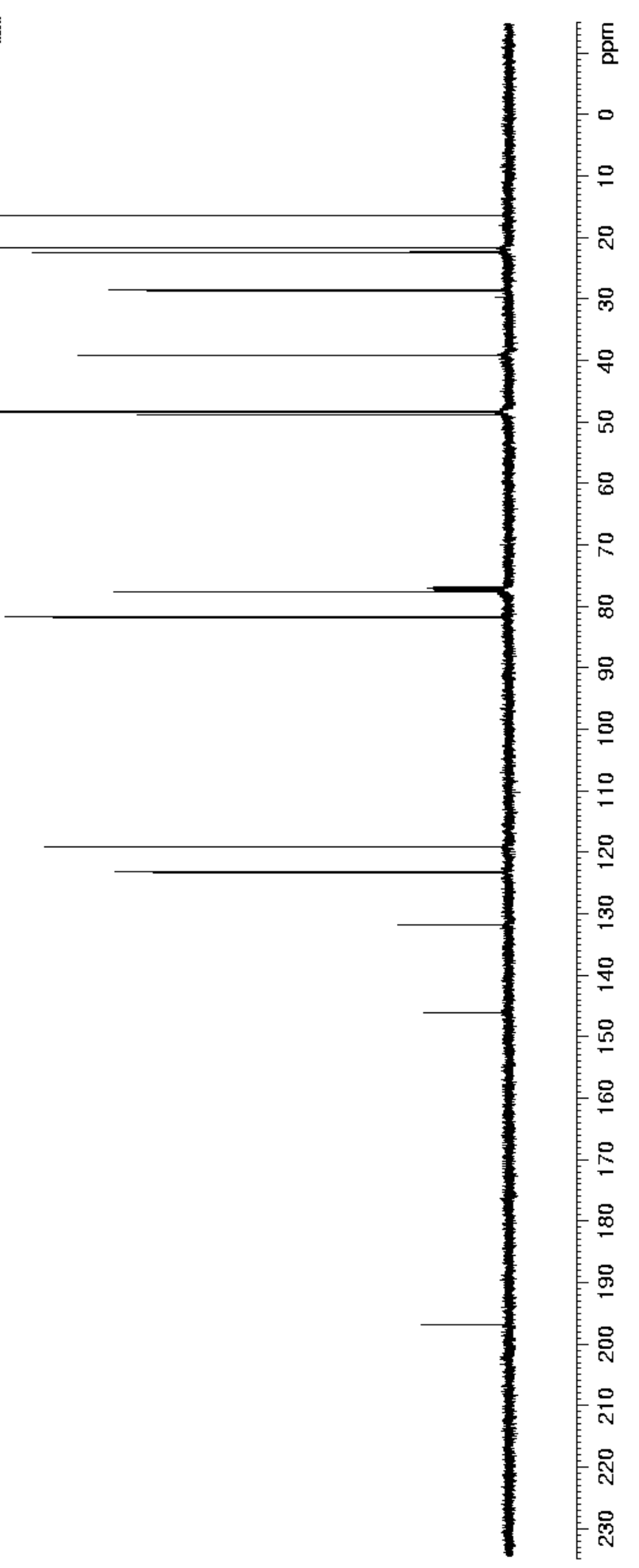



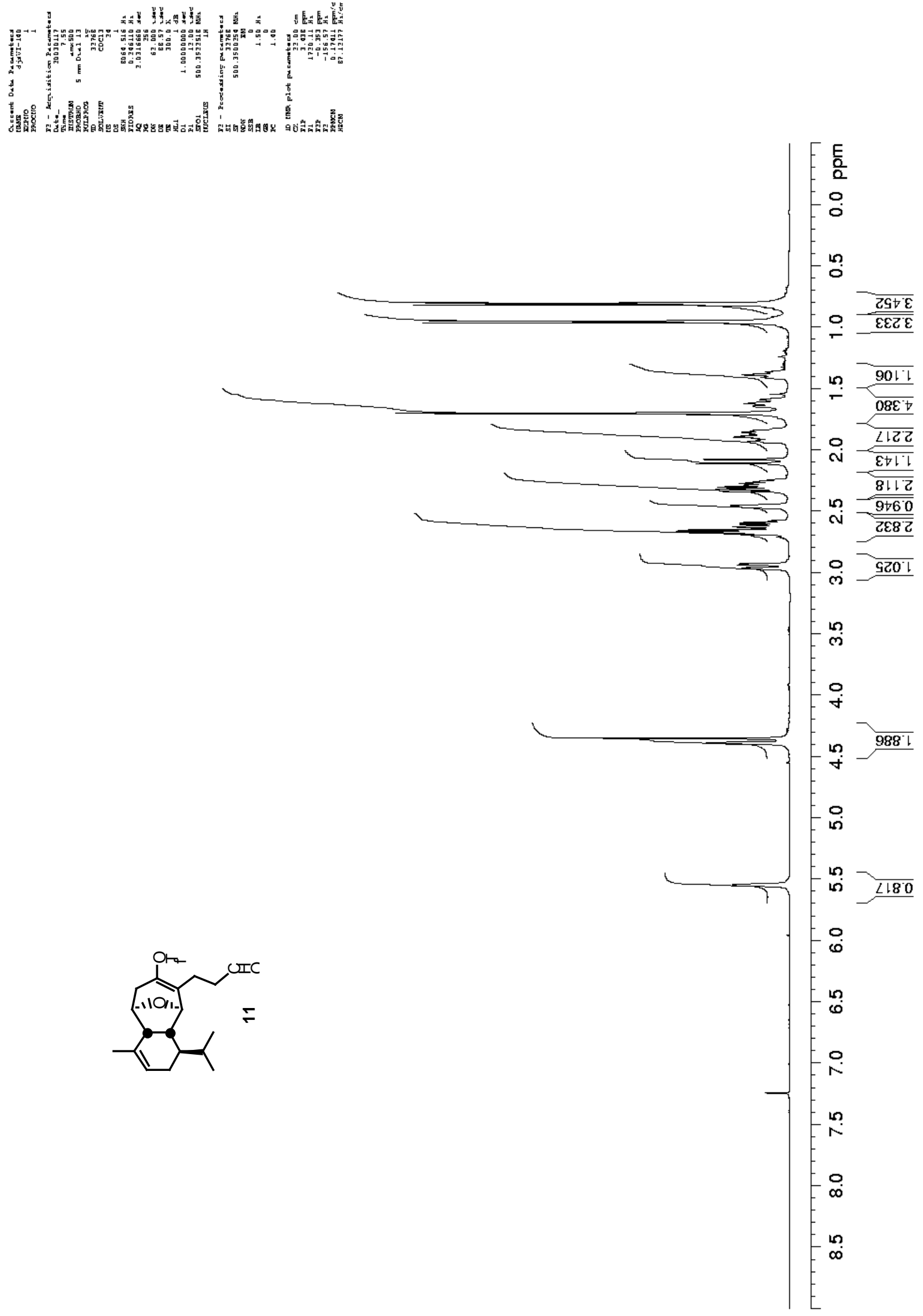

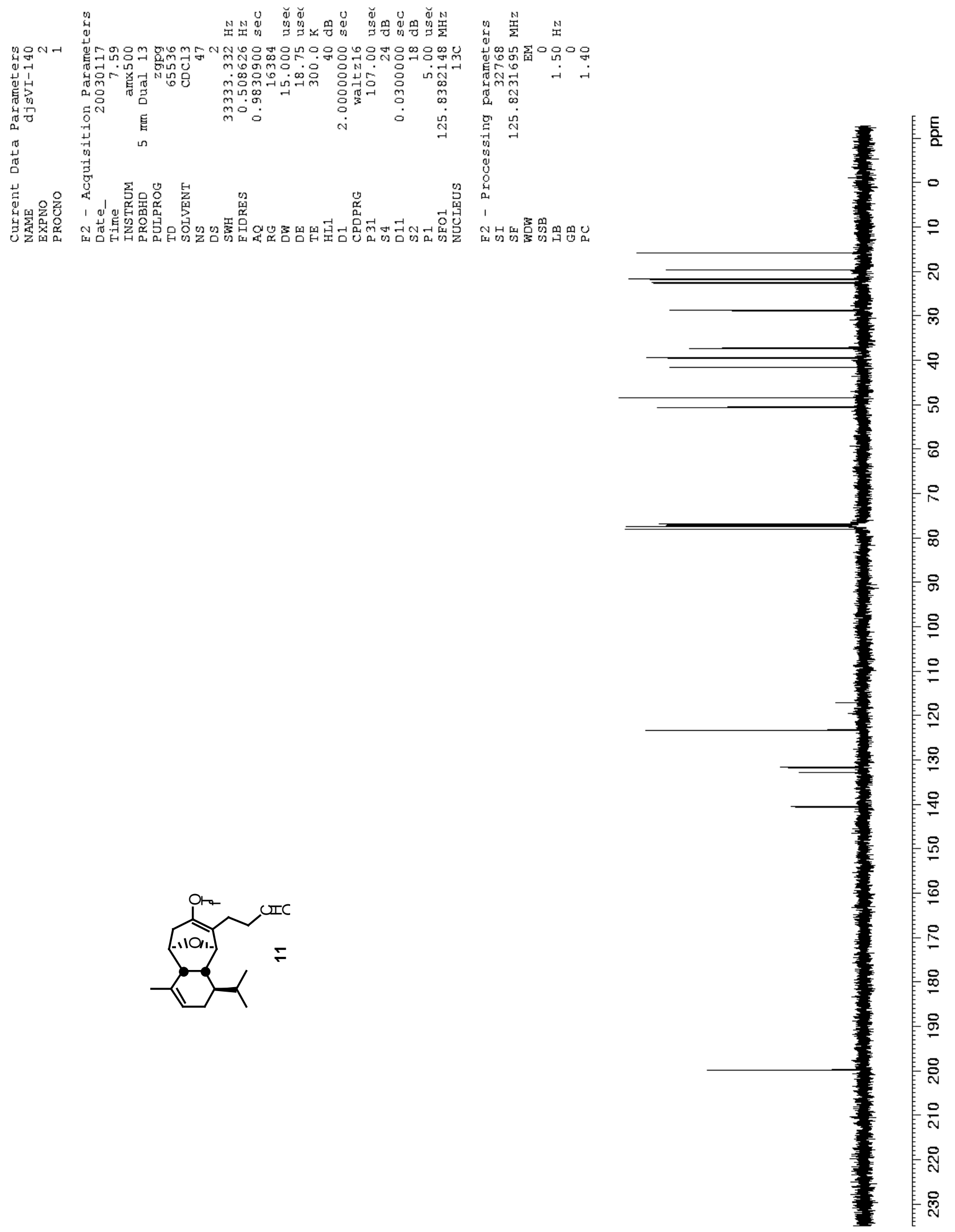


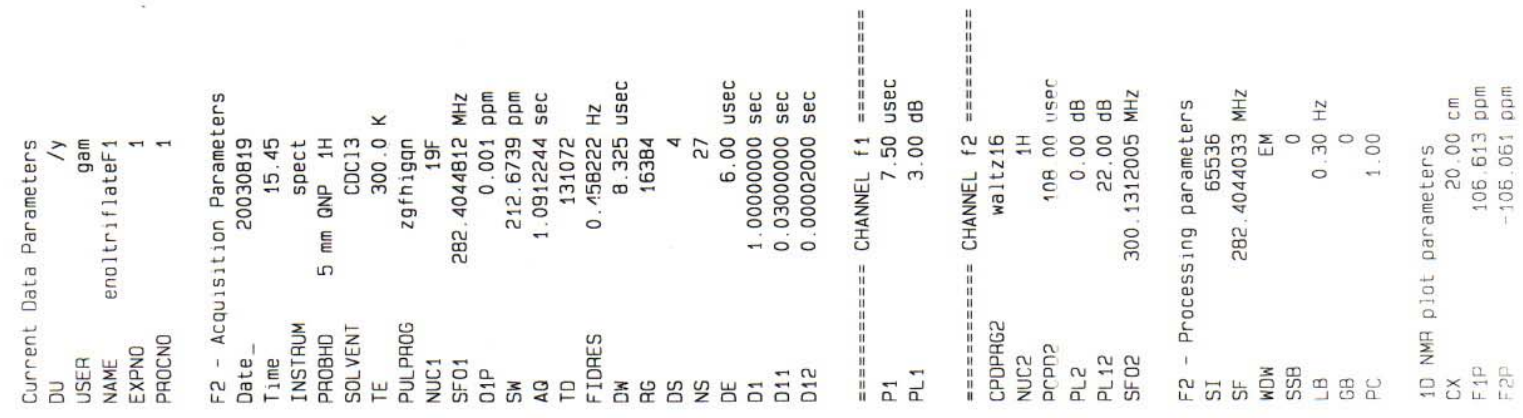

દ६8 $\nabla L-$

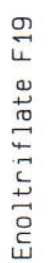

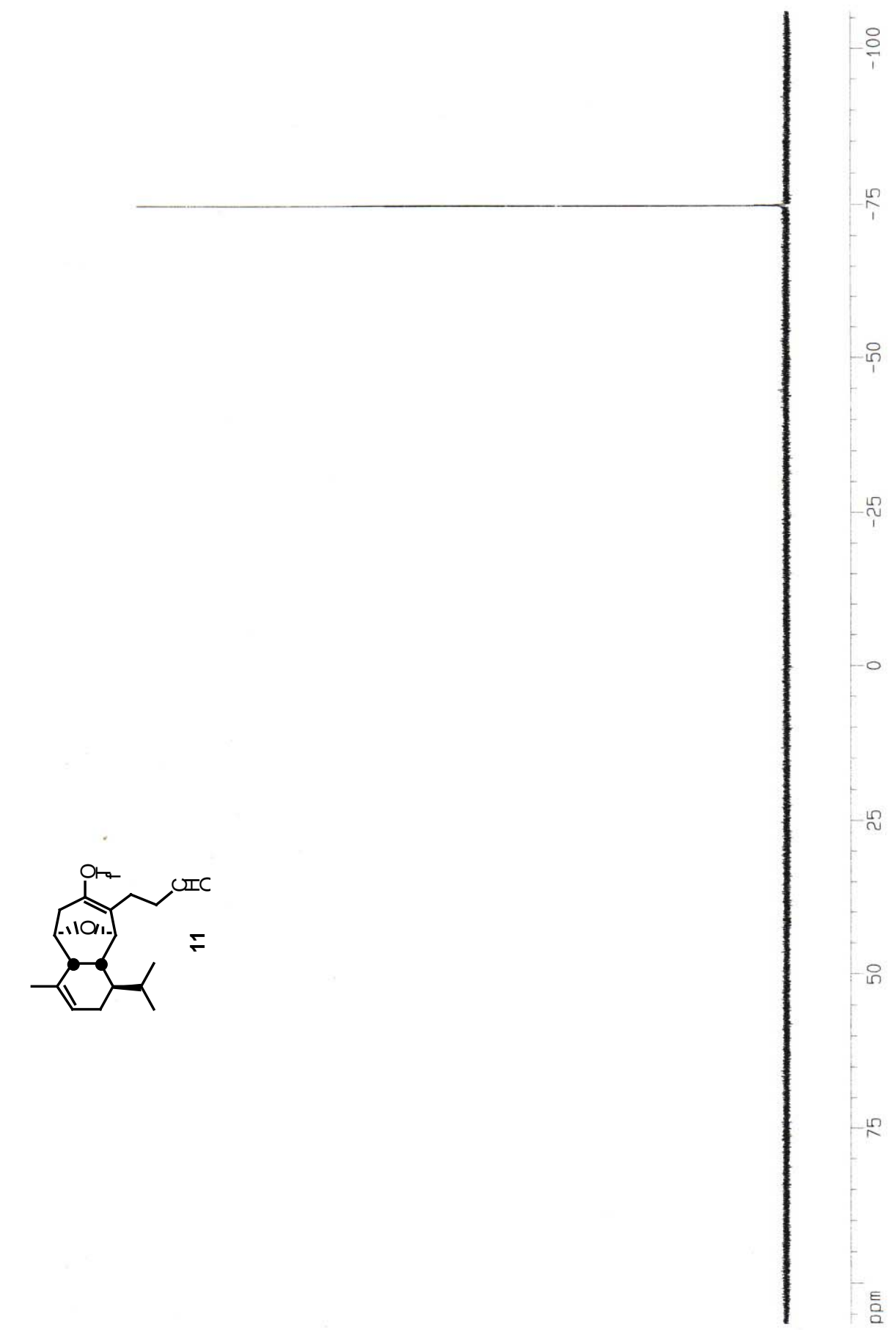



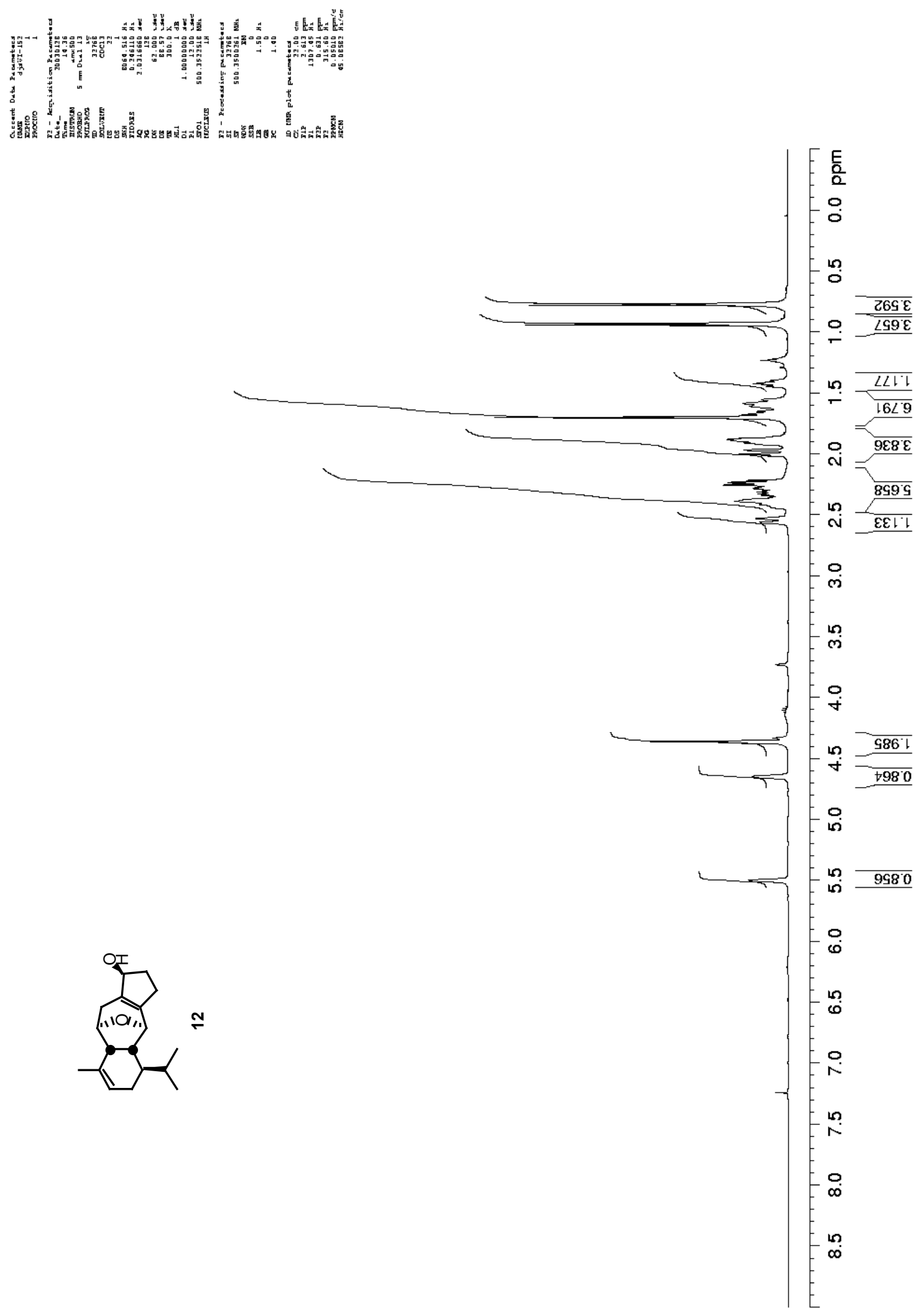

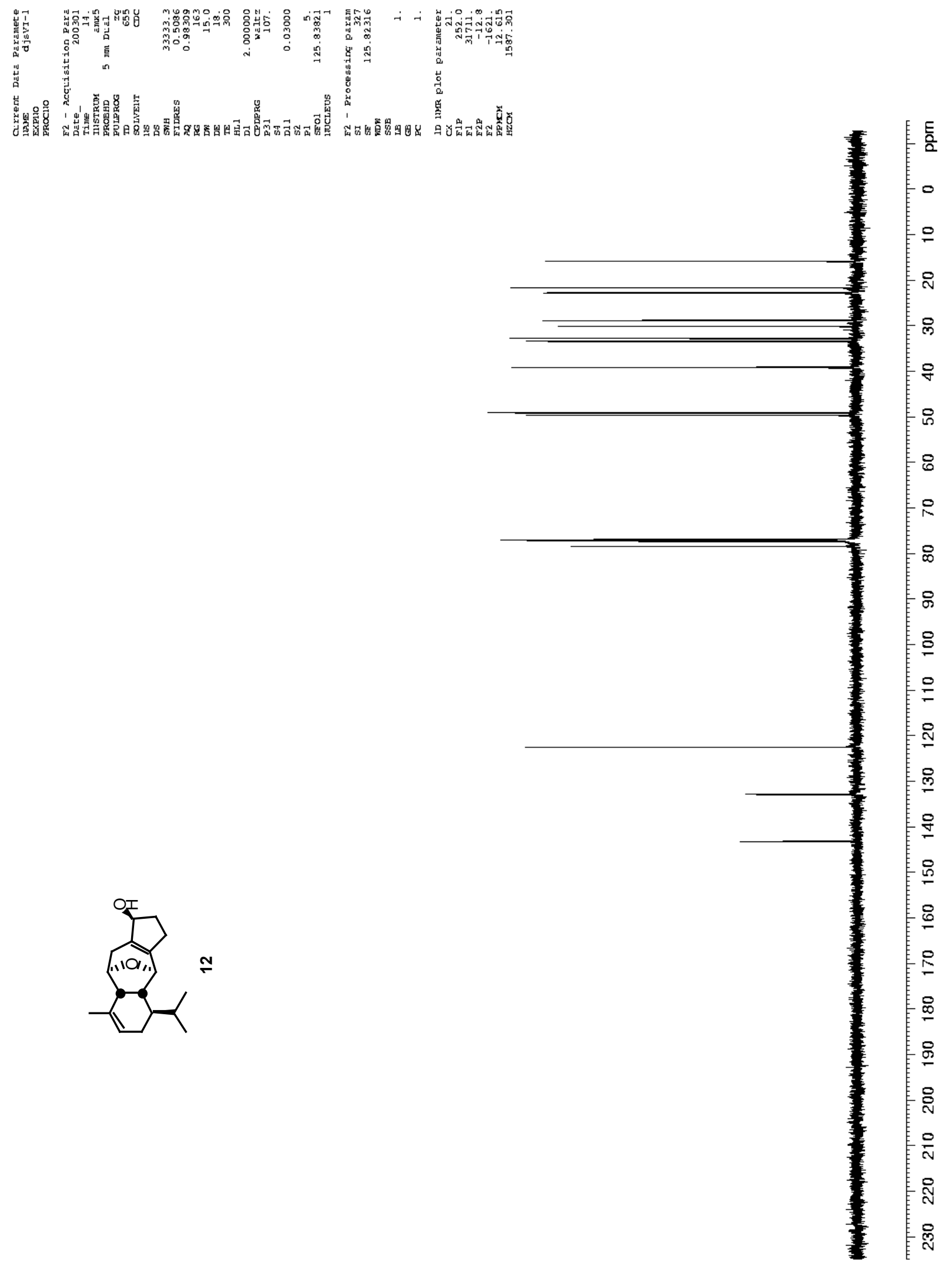

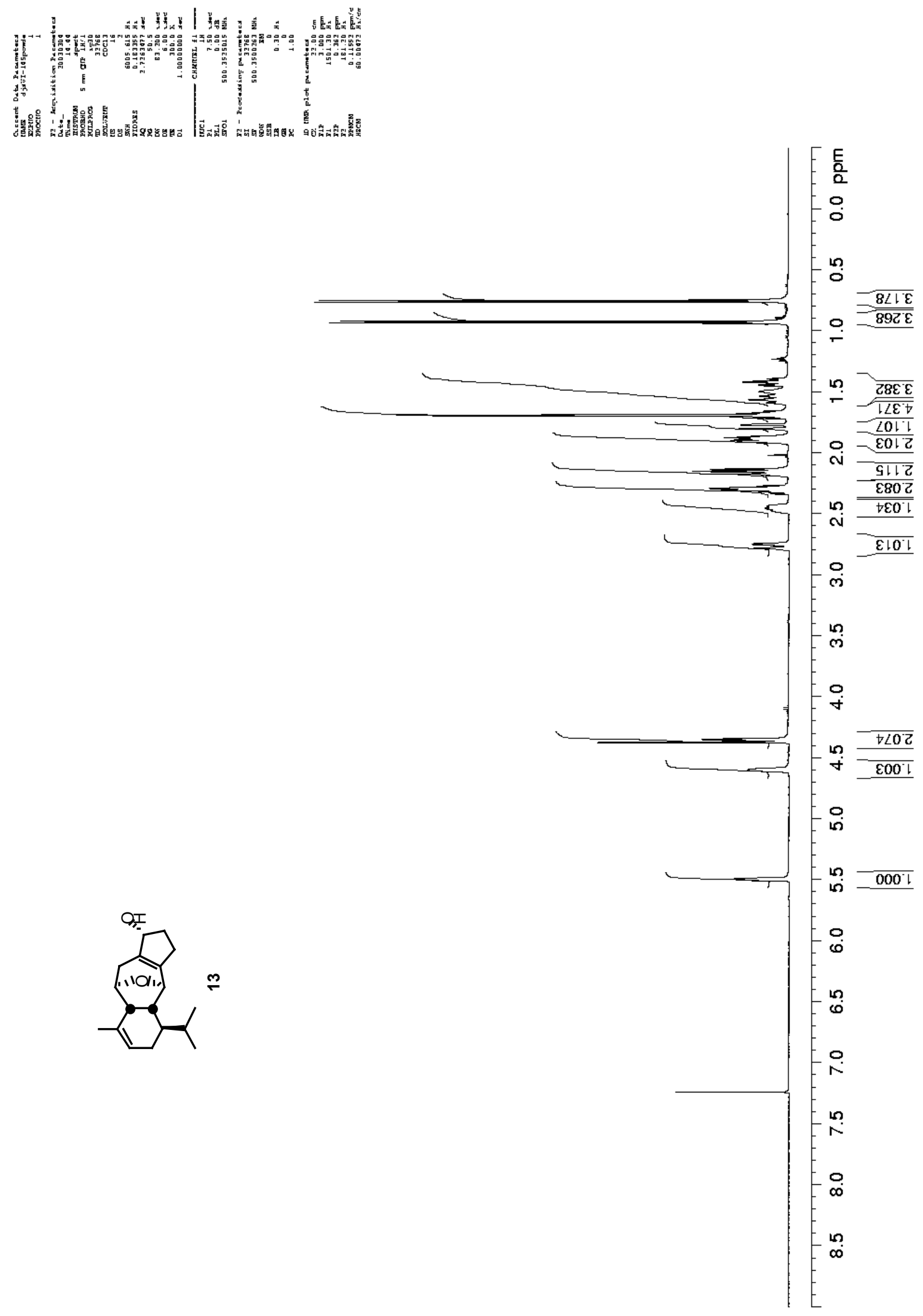

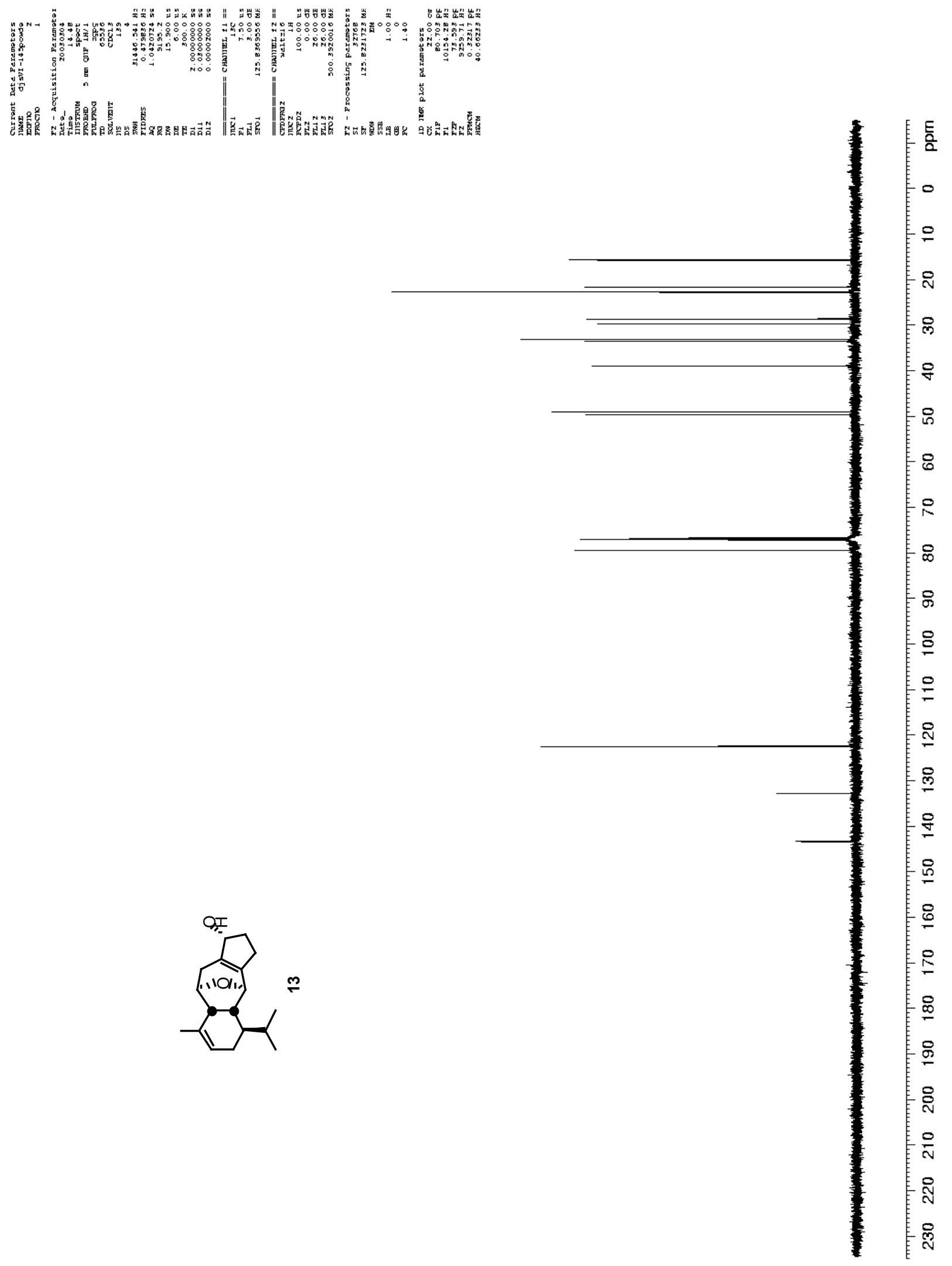

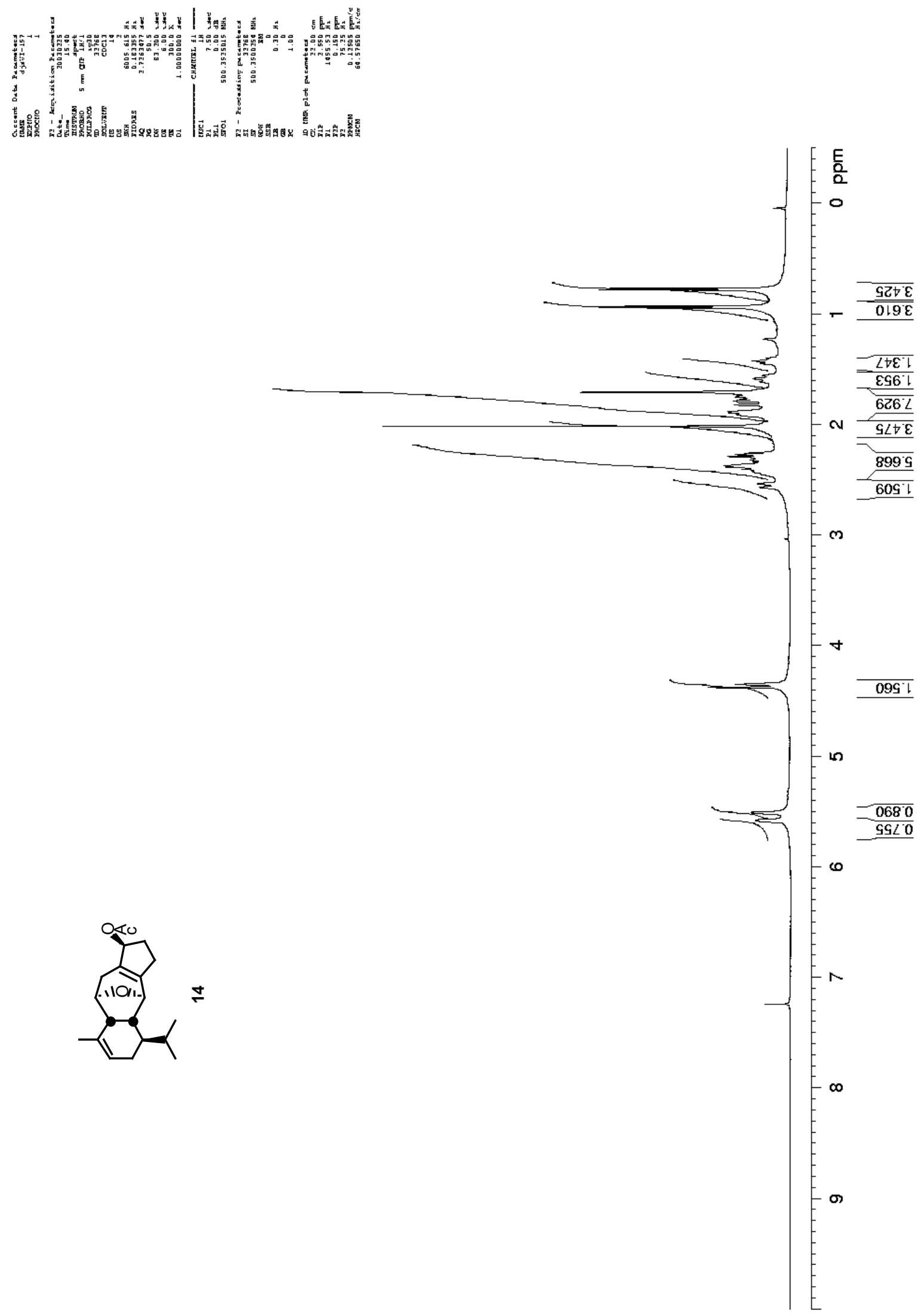

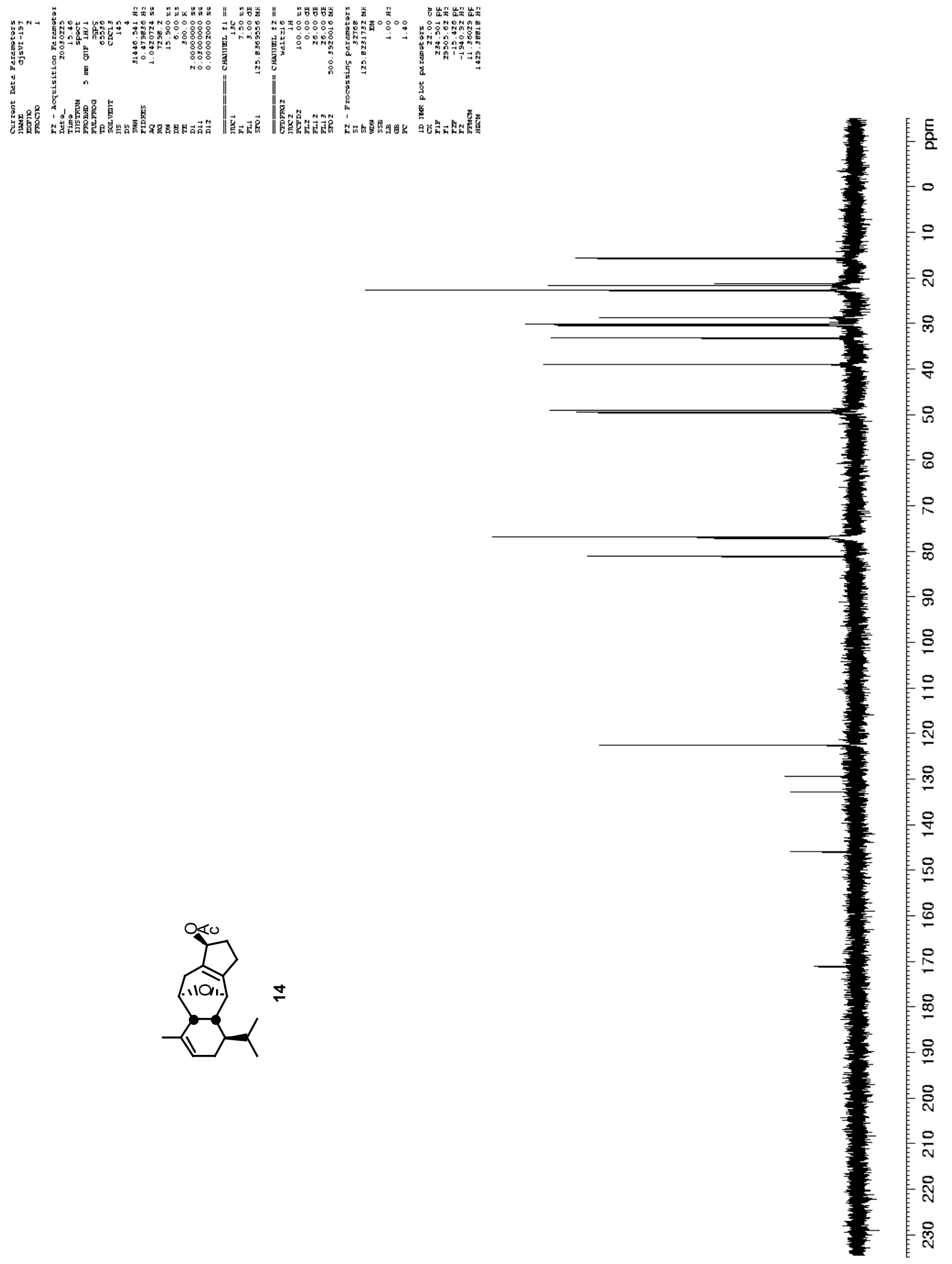

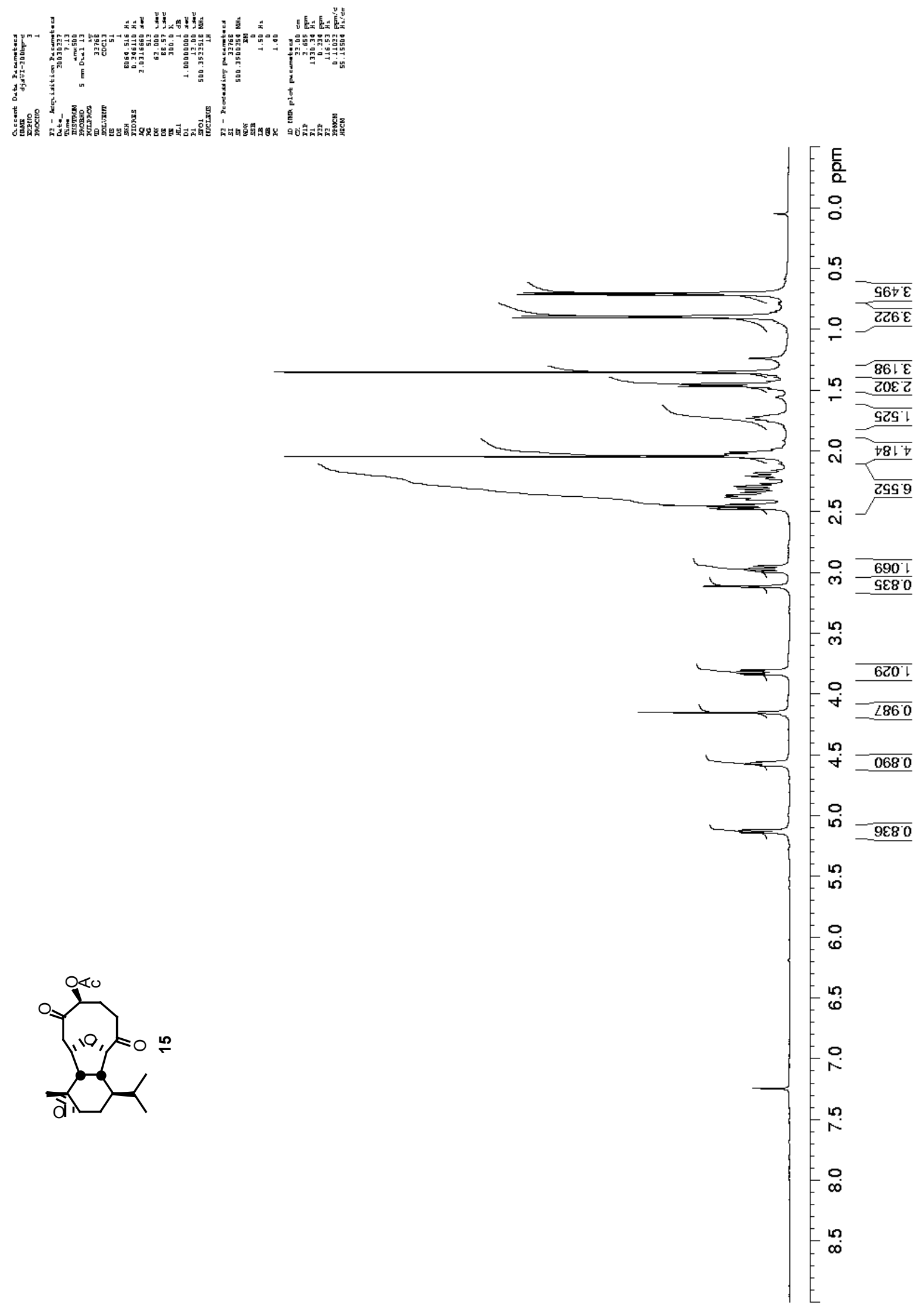

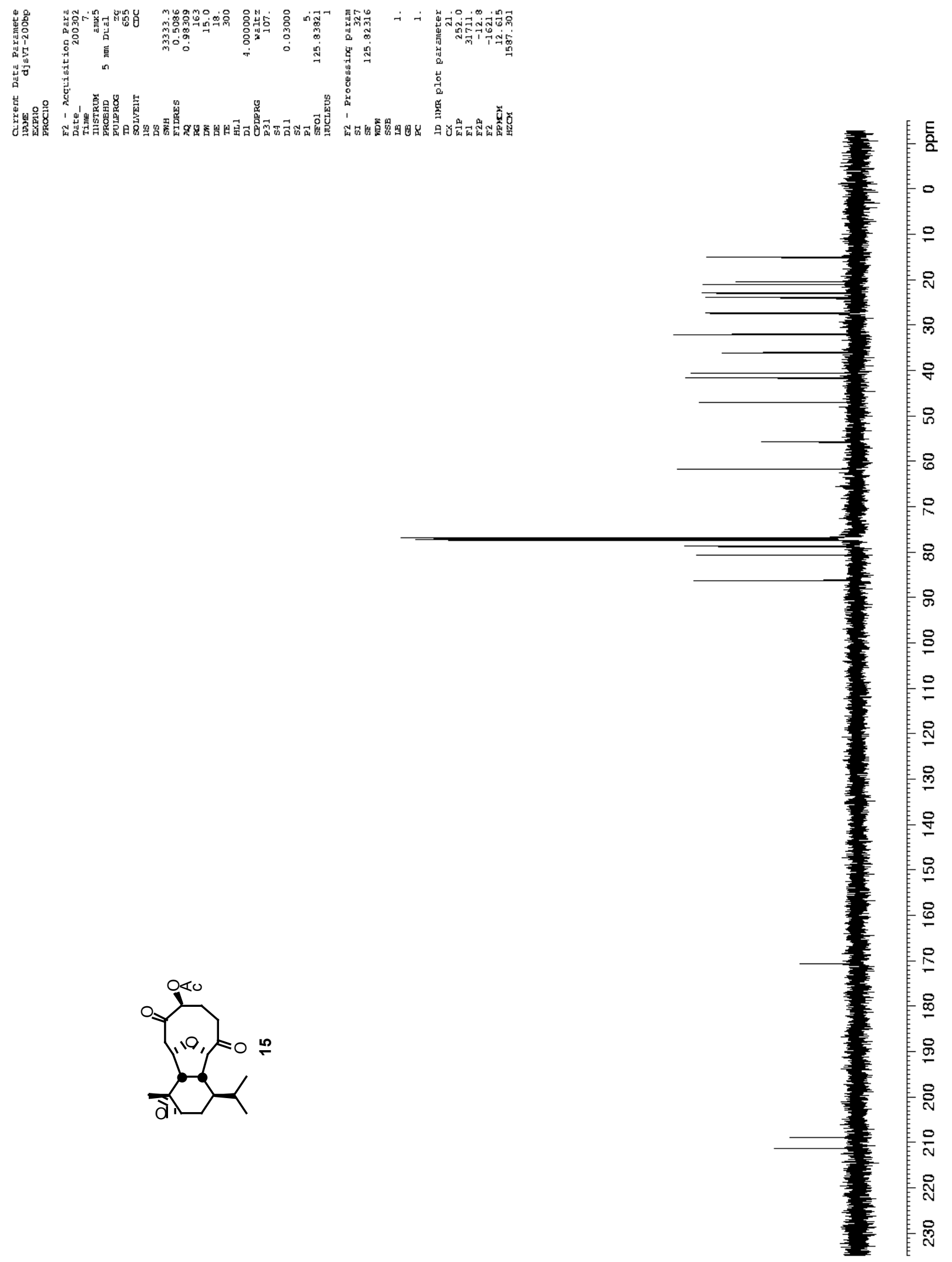

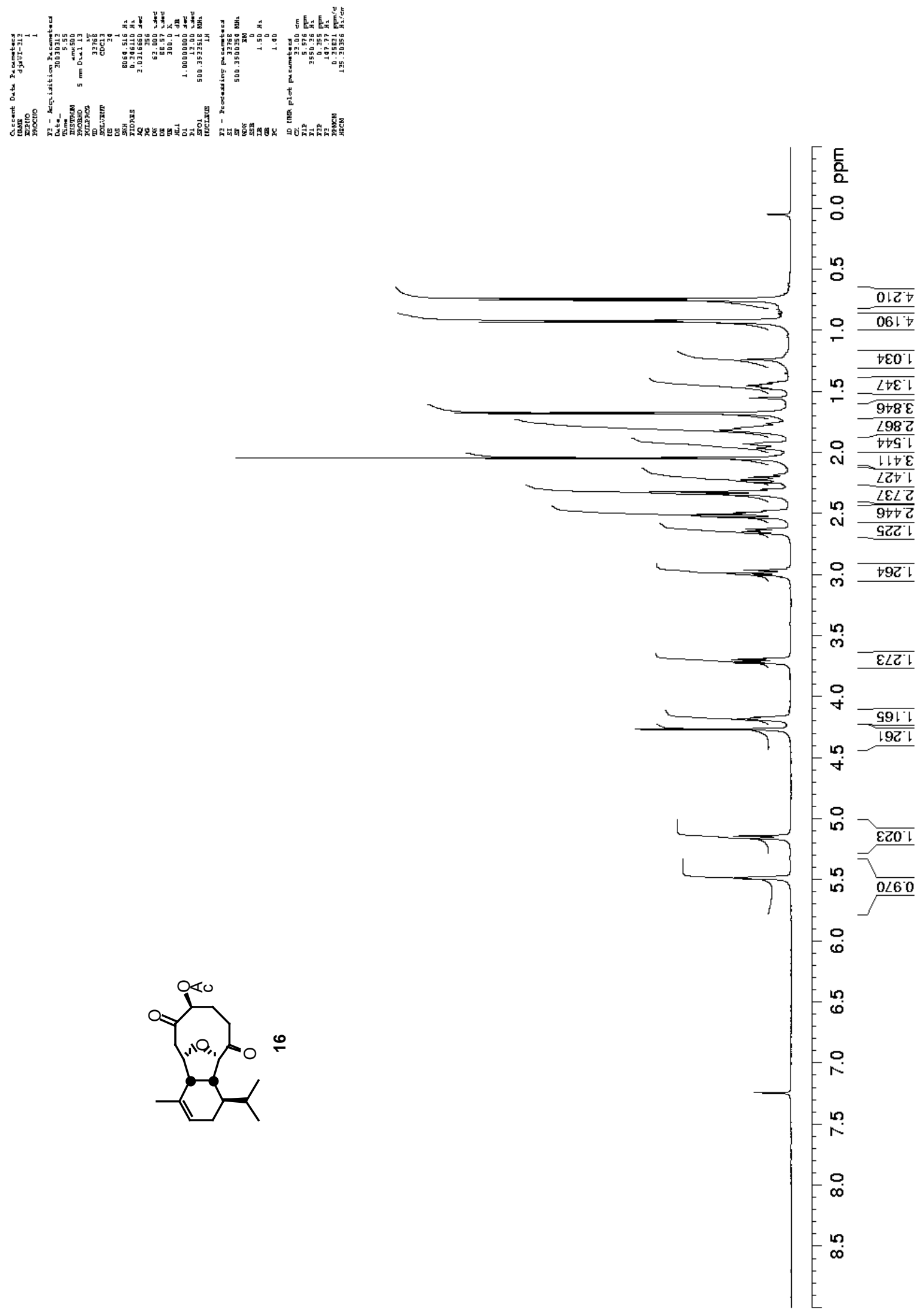

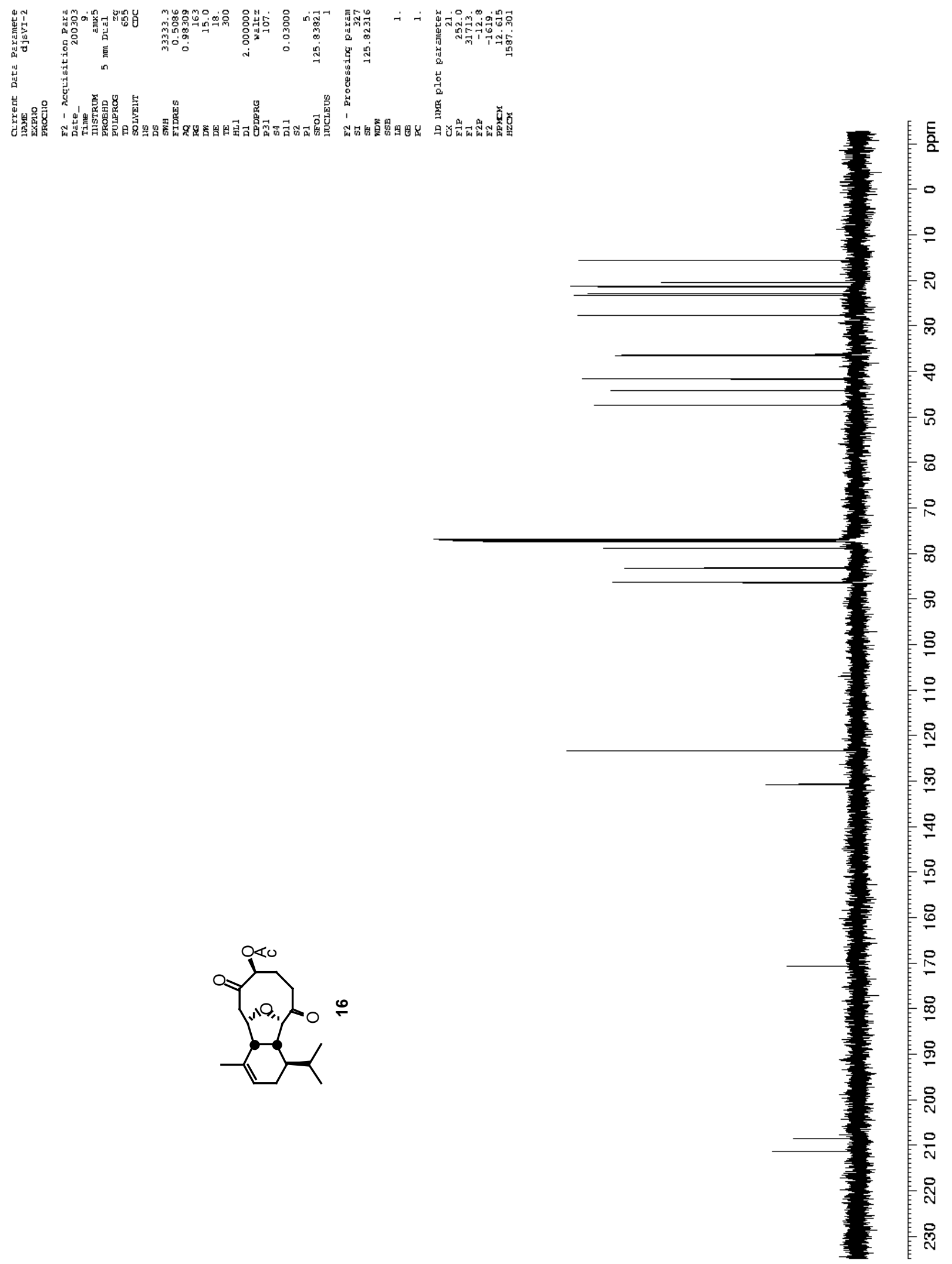

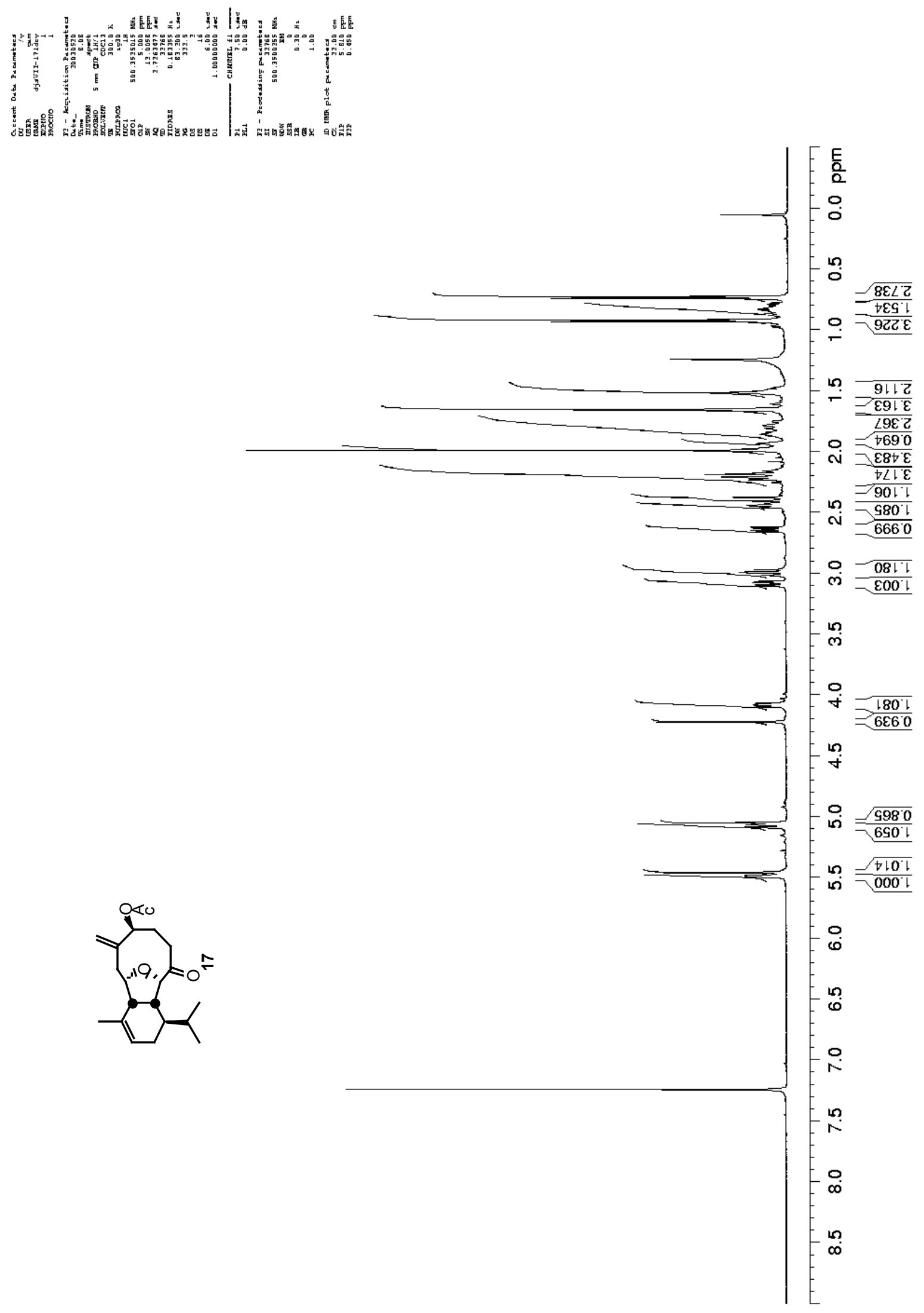

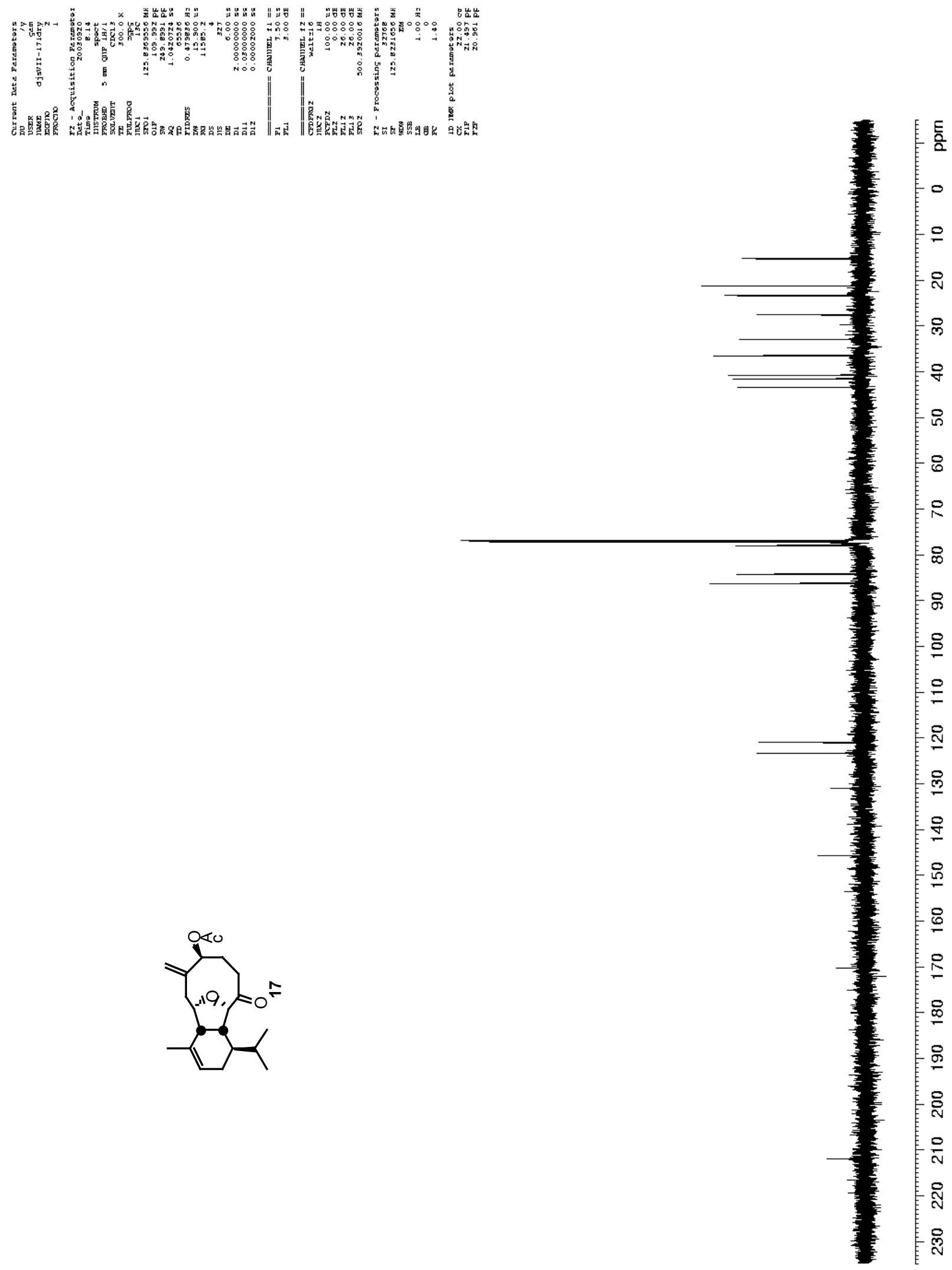

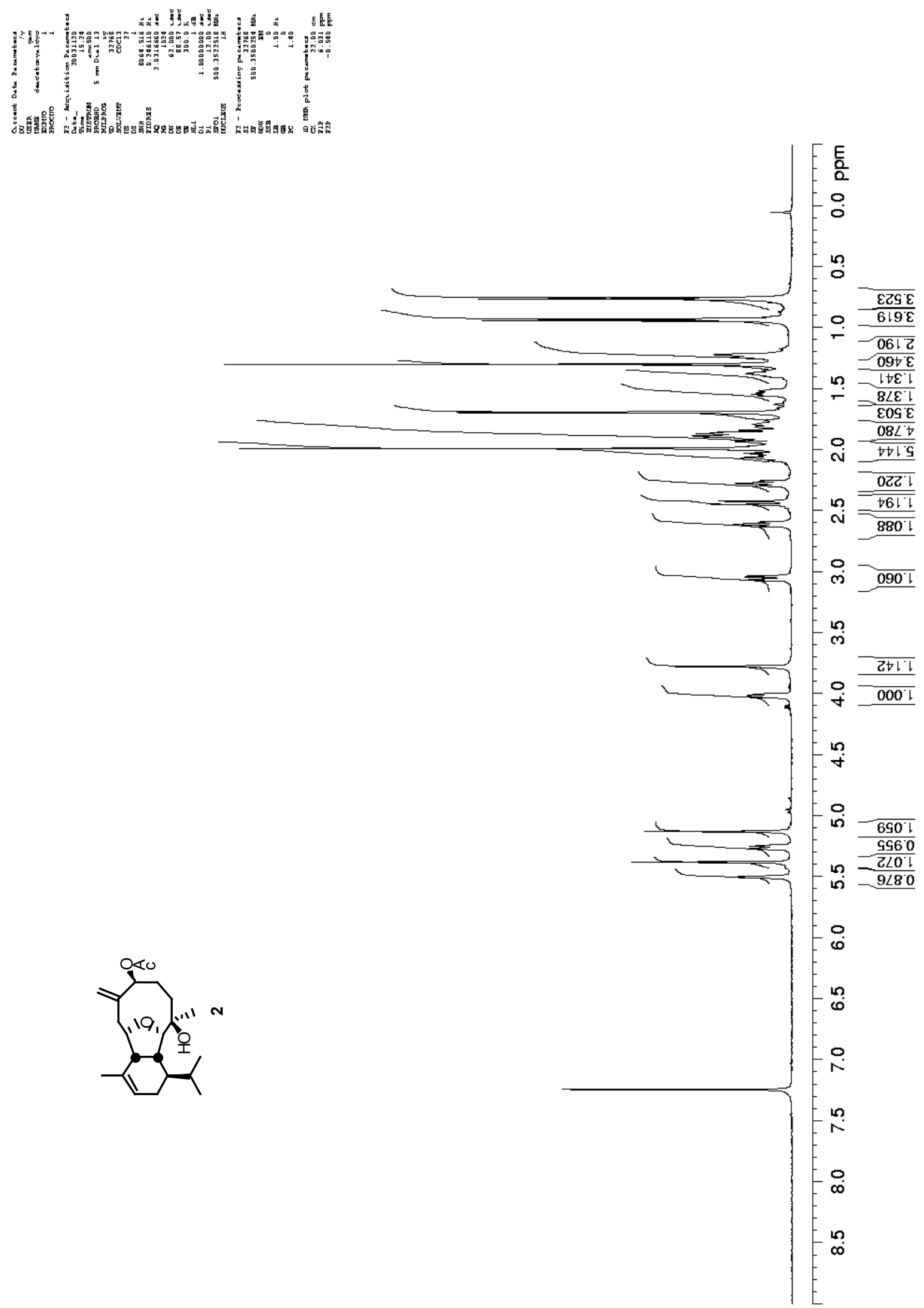

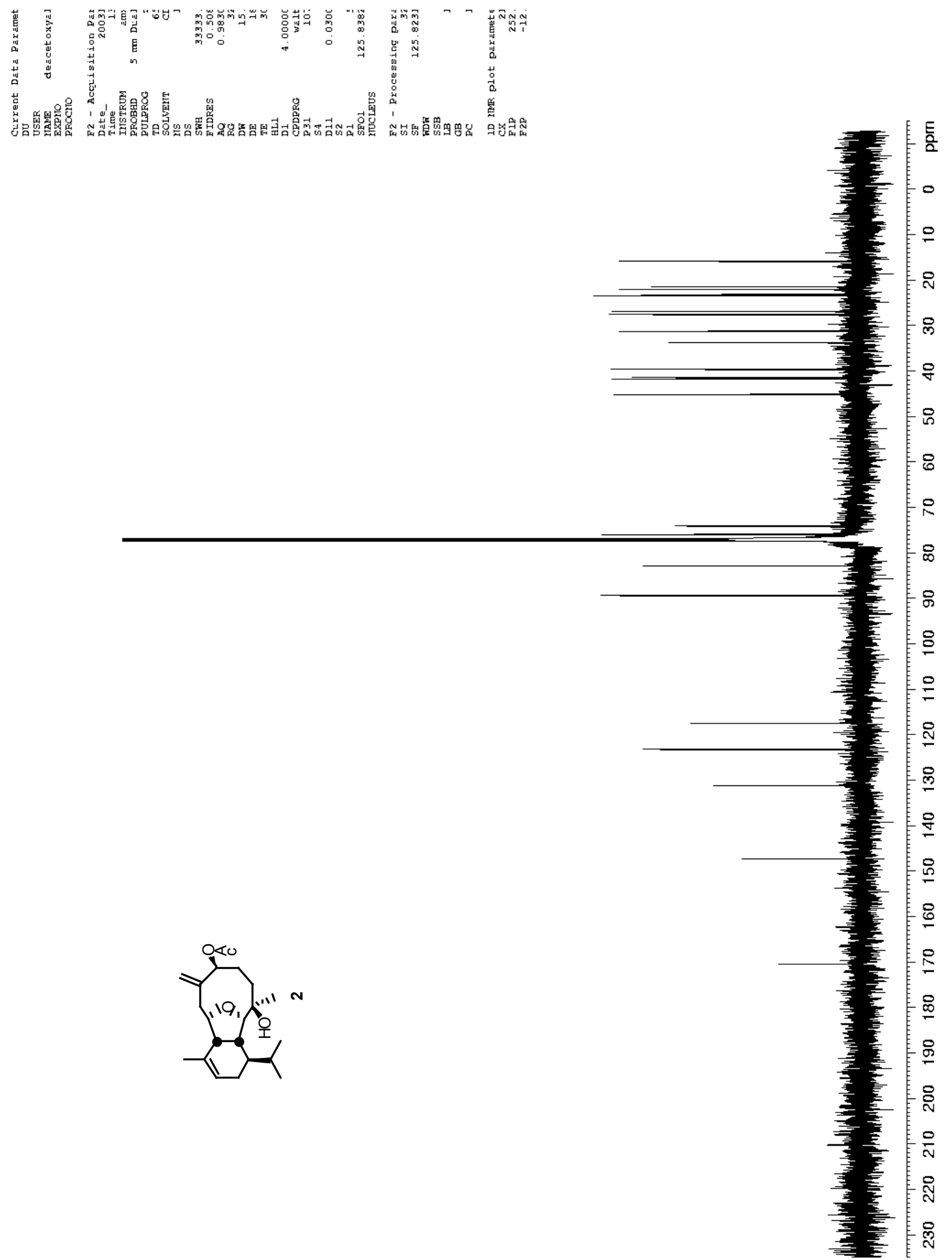


\section{X-ray Structure Determination of Compound 6}

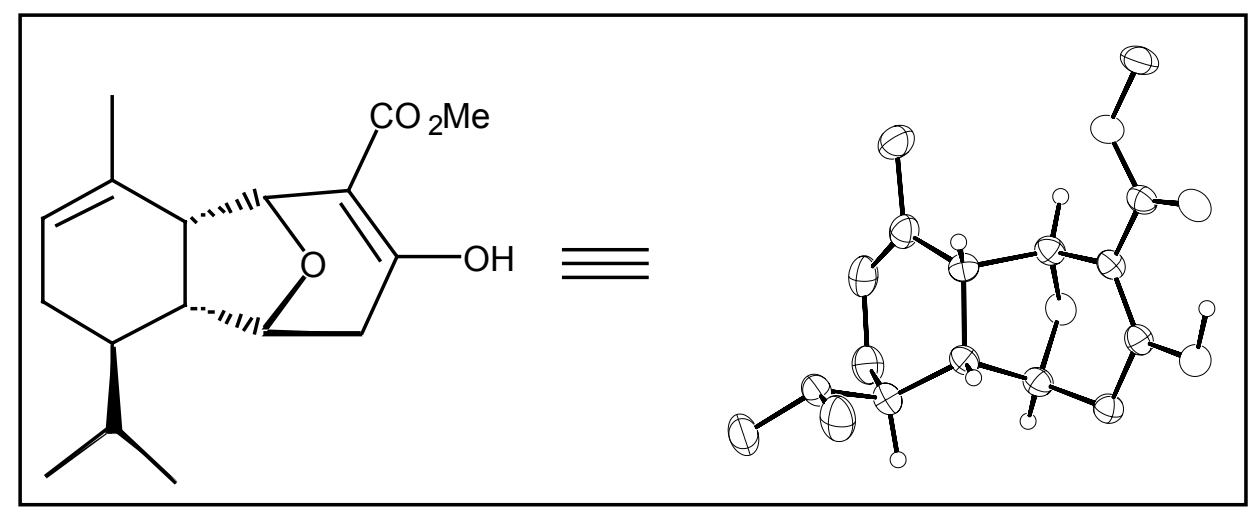

Compound $6, \mathrm{C}_{17} \mathrm{H}_{24} \mathrm{O}_{4}$, crystallizes in the orthorhombic space group $\mathrm{P} 2{ }_{1} 2_{1} 2_{1}$ (systematic absences h00: $\mathrm{h}=$ odd, 0k0: $\mathrm{k}=$ odd, and 001: $\mathrm{l}=$ odd) with $\mathrm{a}=6.5819(2) \AA, \mathrm{b}=11.1635(3) \AA$, $\mathrm{c}=21.5458(8) \AA, \mathrm{V}=1583.12(9) \AA^{3}, \mathrm{Z}=4$ and $\mathrm{d}_{\text {calc }}=1.227 \mathrm{~g} / \mathrm{cm}^{3}$. X-ray intensity data were collected on a Rigaku R-AXIS IIc area detector employing graphite-monochromated Mo- $\mathrm{K}_{\alpha}$ radiation $(\lambda=0.71069 \AA)$ at a temperature of $200^{\circ} \mathrm{K}$. Indexing was performed from a series of $1^{\circ}$ oscillation images with exposures of 150 seconds per frame. A hemisphere of data was collected using $5^{\circ}$ oscillation angles with exposures of 400 seconds per frame and a crystal-to-detector distance of $82 \mathrm{~mm}$. Oscillation images were processed using bioteX ${ }^{5}$, producing a listing of unaveraged $\mathrm{F}^{2}$ and $\sigma\left(\mathrm{F}^{2}\right)$ values which were then passed to the teXsan ${ }^{6}$ program package for further processing and structure solution on a Silicon Graphics Indigo R4000 computer. A total of 9839 reflections were measured over the ranges $5.26 \leq 2 \theta \leq 50.7^{\circ},-7 \leq \mathrm{h} \leq 7,-13 \leq \mathrm{k} \leq 13$, $-25 \leq 1 \leq 25$ yielding 2868 unique reflections $\left(\mathrm{R}_{\mathrm{int}}=0.0496\right)$. The intensity data were corrected for Lorentz and polarization effects but not for absorption.

The structure was solved by direct methods (SIR92 ${ }^{7}$ ). Refinement was by full-matrix least squares based on $\mathrm{F}^{2}$ using SHELXL- $93^{8}$. All reflections were used during refinement $\left(\mathrm{F}^{2}\right.$, s that were experimentally negative were replaced by $\mathrm{F}^{2}=0$ ). The weighting scheme used was $\mathrm{w}=1 /\left[\sigma^{2}\left(\mathrm{~F}_{\mathrm{o}}^{2}\right)+0.0449 \mathrm{P}^{2}+0.4455 \mathrm{P}\right]$ where $\mathrm{P}=\left(\mathrm{F}_{\mathrm{o}}^{2}+2 \mathrm{~F}_{\mathrm{c}}^{2}\right) / 3$. Non-hydrogen atoms were refined anisotropically and hydrogen atoms were refined isotropically. Refinement converged to $\mathrm{R}_{1}=0.0454$ and $\mathrm{wR}_{2}=0.1052$ for 2650 reflections for which $F>4 \sigma(F)$ and $R_{1}=0.0504$, 
$\mathrm{wR}_{2}=0.1090$ and GOF $=1.048$ for all 2868 unique, non-zero reflections and 287 variables ${ }^{9}$. The maximum $\Delta / \sigma$ in the final cycle of least squares was 0.023 and the two most prominent peaks in the final difference Fourier were +0.121 and $-0.139 \mathrm{e} / \AA^{3}$.

Table S1 lists cell information, data collection parameters, and refinement data. Final positional and equivalent isotropic thermal parameters are given in Table S2. Anisotropic thermal parameters are in Table S3. Tables S4 and S5 list bond distances and bond angles. Figure 1. is an ORTEP ${ }^{10}$ representation of the molecule with $30 \%$ probability thermal ellipsoids displayed.

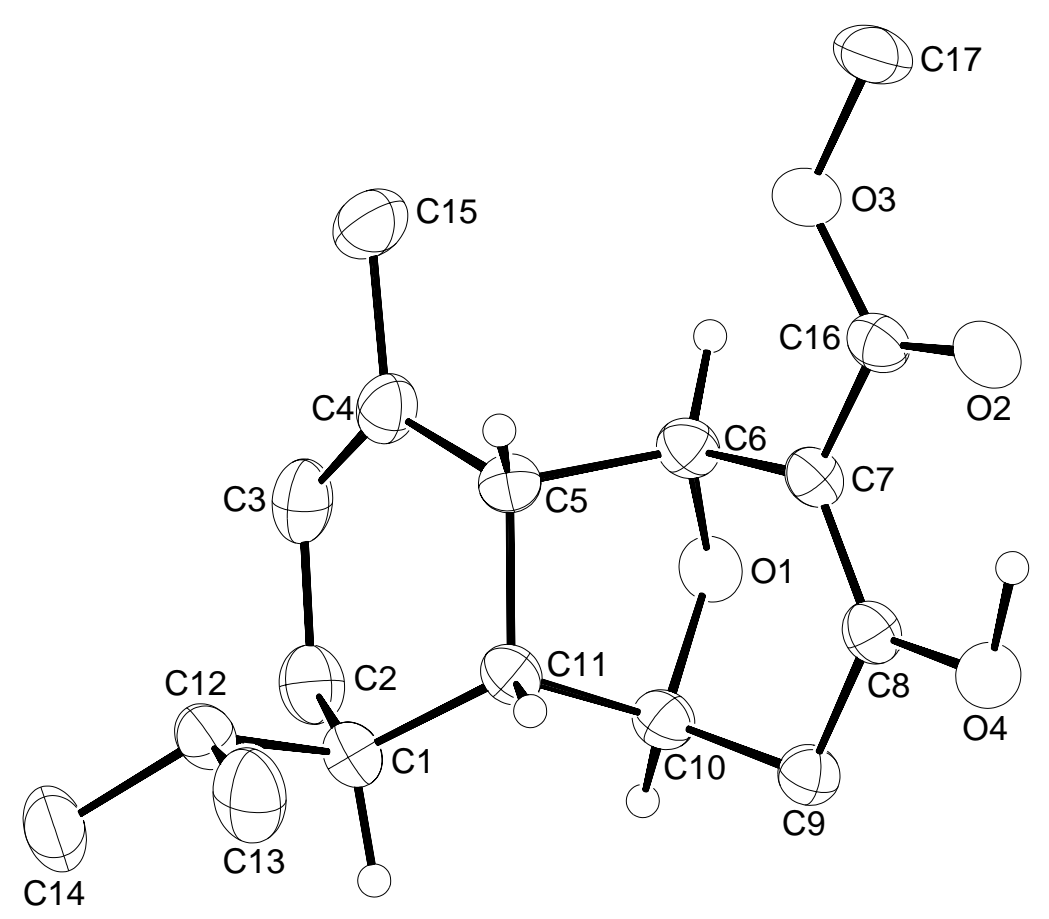

Figure S1. ORTEP drawing of the title compound with $30 \%$ probability thermal ellipsoids. 


\section{Table S1. Summary of Structure Determination of Compound 6}

Formula:

Formula weight:

Crystal class:

Space group:

$\mathrm{Z}$

Cell constants:

$\quad \mathrm{a}$

$\mathrm{b}$
$\mathrm{c}$
$\mathrm{V}$
$\mu$
crystal size, $\mathrm{mm}$
$\mathrm{D}_{\text {calc }}$

$\mathrm{F}(000)$

Radiation:

$2 \theta$ range

hkl collected:

No. reflections measured:

No. unique reflections:

No. observed reflections

No. reflections used in refinement

No. parameters

$R$ indices $(F>4 \sigma)$

$\mathrm{R}$ indices (all data)

GOF:

Final Difference Peaks, e/ $\AA^{3}$
$\mathrm{C}_{17} \mathrm{H}_{24} \mathrm{O}_{4}$

292.36

orthorhombic

$\mathrm{P} 2{ }_{1} 2_{1} 2_{1}$ (\#19)

4

$6.5819(2) \AA$

$11.1635(3) \AA$

$21.5458(8) \AA$

$1583.12(9) \AA^{3}$

$0.86 \mathrm{~cm}^{-1}$

$0.30 \times 0.12 \times 0.10$

$1.227 \mathrm{~g} / \mathrm{cm}^{3}$

632

Mo-K $\mathrm{K}_{\alpha}(\lambda=0.71069 \AA)$

$5.26-50.7^{\circ}$

$-7 \leq \mathrm{h} \leq 7 ;-13 \leq \mathrm{k} \leq 13 ;-25 \leq 1 \leq 25$

9839

$2868\left(\mathrm{R}_{\text {int }}=0.0496\right)$

$2650(\mathrm{~F}>4 \sigma)$

2868

287

$\mathrm{R}_{1}=0.0454$

$\mathrm{wR}_{2}=0.1052$

$\mathrm{R}_{1}=0.0504$

$\mathrm{wR}_{2}=0.1090$

1.048

$+0.121,-0.139$ 
Table S2. Refined Positional Parameters for Compound 6

\begin{tabular}{|c|c|c|c|c|}
\hline Atom & $\mathrm{x}$ & $\mathrm{y}$ & $\mathrm{Z}$ & $\mathrm{U}_{\mathrm{eq}}, \AA^{2}$ \\
\hline C1 & $0.6755(4)$ & $0.5090(2)$ & $0.64443(12)$ & $0.0470(6)$ \\
\hline $\mathrm{C} 2$ & $0.8921(4)$ & $0.5596(3)$ & $0.64544(14)$ & $0.0569(7)$ \\
\hline C3 & $0.9064(4)$ & $0.6678(3)$ & $0.60541(13)$ & $0.0595(7)$ \\
\hline C4 & $0.7529(4)$ & $0.7417(2)$ & $0.59460(11)$ & $0.0514(6)$ \\
\hline C5 & $0.5483(4)$ & $0.7251(2)$ & $0.62552(11)$ & $0.0428(5)$ \\
\hline C6 & $0.5188(4)$ & $0.8193(2)$ & $0.67763(11)$ & $0.0429(5)$ \\
\hline $\mathrm{C} 7$ & $0.2984(3)$ & $0.8298(2)$ & $0.69785(10)$ & $0.0402(5)$ \\
\hline $\mathrm{C} 8$ & $0.2292(3)$ & $0.7527(2)$ & $0.74155(10)$ & $0.0418(5)$ \\
\hline C9 & $0.3591(4)$ & $0.6552(2)$ & $0.76720(12)$ & $0.0447(5)$ \\
\hline C10 & $0.5577(4)$ & $0.6497(2)$ & $0.73143(11)$ & $0.0432(5)$ \\
\hline C11 & $0.5278(4)$ & $0.6080(2)$ & $0.66353(11)$ & $0.0416(5)$ \\
\hline C12 & $0.6230(4)$ & $0.4505(2)$ & $0.58097(13)$ & $0.0518(6)$ \\
\hline C13 & $0.4040(6)$ & $0.4079(4)$ & $0.5784(2)$ & $0.0792(10)$ \\
\hline C14 & $0.7668(7)$ & $0.3471(4)$ & $0.5667(2)$ & $0.0797(10)$ \\
\hline C15 & $0.7692(6)$ & $0.8480(3)$ & $0.5520(2)$ & $0.0674(8)$ \\
\hline C16 & $0.1671(4)$ & $0.9228(2)$ & $0.67412(11)$ & $0.0441(5)$ \\
\hline C17 & $0.1432(6)$ & $1.0916(3)$ & $0.6081(2)$ & $0.0645(8)$ \\
\hline $\mathrm{O} 1$ & $0.6357(2)$ & $0.77005(14)$ & $0.72741(7)$ & $0.0448(4)$ \\
\hline $\mathrm{O} 2$ & $-0.0077(3)$ & $0.9416(2)$ & $0.69092(8)$ & $0.0538(5)$ \\
\hline $\mathrm{O} 3$ & $0.2590(3)$ & $0.9907(2)$ & $0.63067(8)$ & $0.0534(4)$ \\
\hline $\mathrm{O} 4$ & $0.0408(2)$ & $0.7544(2)$ & $0.76571(8)$ & $0.0494(4)$ \\
\hline $\mathrm{H} 1$ & $0.660(4)$ & $0.441(3)$ & $0.6766(13)$ & $0.058(8)$ \\
\hline $\mathrm{H} 2 \mathrm{~b}$ & $0.998(5)$ & $0.497(3)$ & $0.6299(14)$ & $0.073(9)$ \\
\hline $\mathrm{H} 2 \mathrm{a}$ & $0.936(5)$ & $0.580(3)$ & $0.692(2)$ & $0.084(10)$ \\
\hline $\mathrm{H} 3$ & $1.037(5)$ & $0.688(3)$ & $0.5832(14)$ & $0.074(9)$ \\
\hline $\mathrm{H} 4$ & $-0.042(6)$ & $0.820(3)$ & $0.740(2)$ & $0.096(11)$ \\
\hline $\mathrm{H} 5$ & $0.433(4)$ & $0.739(2)$ & $0.5946(12)$ & $0.050(7)$ \\
\hline $\mathrm{H} 6$ & $0.563(4)$ & $0.902(2)$ & $0.6681(11)$ & $0.041(6)$ \\
\hline $\mathrm{H} 9 \mathrm{~b}$ & $0.380(4)$ & $0.672(3)$ & $0.8086(13)$ & $0.057(7)$ \\
\hline $\mathrm{H} 9 \mathrm{a}$ & $0.281(4)$ & $0.576(2)$ & $0.7647(11)$ & $0.042(6)$ \\
\hline $\mathrm{H} 10$ & $0.658(4)$ & $0.604(2)$ & $0.7551(12)$ & $0.049(7)$ \\
\hline $\mathrm{H} 11$ & $0.395(4)$ & $0.578(2)$ & $0.6594(10)$ & $0.030(5)$ \\
\hline $\mathrm{H} 12$ & $0.642(4)$ & $0.514(3)$ & $0.5491(13)$ & $0.054(7)$ \\
\hline $\mathrm{H} 13 \mathrm{a}$ & $0.294(9)$ & $0.483(5)$ & $0.581(3)$ & $0.16(2)$ \\
\hline $\mathrm{H} 13 \mathrm{~b}$ & $0.392(6)$ & $0.355(3)$ & $0.610(2)$ & $0.079(12)$ \\
\hline $\mathrm{H} 13 \mathrm{c}$ & $0.375(6)$ & $0.358(4)$ & $0.537(2)$ & $0.100(12)$ \\
\hline $\mathrm{H} 14 \mathrm{c}$ & $0.720(5)$ & $0.298(3)$ & $0.529(2)$ & $0.098(12)$ \\
\hline $\mathrm{H} 14 \mathrm{a}$ & $0.767(7)$ & $0.292(4)$ & $0.604(2)$ & $0.14(2)$ \\
\hline $\mathrm{H} 14 \mathrm{~b}$ & $0.906(8)$ & $0.382(4)$ & $0.555(2)$ & $0.12(2)$ \\
\hline $\mathrm{H} 15 \mathrm{~b}$ & $0.672(6)$ & $0.845(3)$ & $0.517(2)$ & $0.092(12)$ \\
\hline $\mathrm{H} 15 \mathrm{c}$ & $0.917(6)$ & $0.856(3)$ & $0.534(2)$ & $0.080(10)$ \\
\hline $\mathrm{H} 15 \mathrm{a}$ & $0.737(5)$ & $0.925(3)$ & $0.5721(14)$ & $0.063(8)$ \\
\hline $\mathrm{H} 17 \mathrm{c}$ & $0.092(6)$ & $1.143(3)$ & $0.643(2)$ & $0.103(12)$ \\
\hline $\mathrm{H} 17 \mathrm{a}$ & $0.025(7)$ & $1.060(3)$ & $0.586(2)$ & $0.109(13)$ \\
\hline $\mathrm{H} 17 \mathrm{~b}$ & $0.240(6)$ & $1.130(3)$ & $0.578(2)$ & $0.090(11)$ \\
\hline
\end{tabular}

Table S3. Refined Thermal Parameters (U's) for Compound 6 


\begin{tabular}{|c|c|c|c|c|c|c|}
\hline Atom & $\mathrm{U}_{11}$ & $\mathrm{U}_{22}$ & $\mathrm{U}_{33}$ & $\mathrm{U}_{23}$ & $\mathrm{U}_{13}$ & $\mathrm{U}_{12}$ \\
\hline C1 & $0.0478(13)$ & $0.0420(12)$ & $0.0512(14)$ & $-0.0045(11)$ & $0.0015(11)$ & $0.0058(11)$ \\
\hline $\mathrm{C} 2$ & $0.0414(14)$ & $0.066(2)$ & $0.063(2)$ & $-0.0073(14)$ & $0.0009(13)$ & $0.0089(13)$ \\
\hline C3 & $0.047(2)$ & $0.072(2)$ & $0.059(2)$ & $-0.0173(14)$ & $0.0104(13)$ & $-0.0047(14)$ \\
\hline C4 & $0.055(2)$ & $0.0522(14)$ & $0.0474(12)$ & $-0.0083(11)$ & $0.0076(12)$ & $-0.0100(13)$ \\
\hline C5 & $0.0431(12)$ & $0.0420(12)$ & $0.0434(11)$ & $0.0022(10)$ & $0.0001(11)$ & $-0.0020(10)$ \\
\hline C6 & $0.0402(12)$ & $0.0388(12)$ & $0.0498(12)$ & $-0.0006(10)$ & $-0.0004(11)$ & $-0.0031(10)$ \\
\hline $\mathrm{C} 7$ & $0.0381(11)$ & $0.0346(11)$ & $0.0479(12)$ & $-0.0035(10)$ & $-0.0004(10)$ & $0.0003(9)$ \\
\hline $\mathrm{C} 8$ & $0.0397(11)$ & $0.0389(11)$ & $0.0466(11)$ & $-0.0049(9)$ & $-0.0017(10)$ & $-0.0004(10)$ \\
\hline C9 & $0.0468(13)$ & $0.0429(12)$ & $0.0445(13)$ & $0.0014(11)$ & $-0.0013(11)$ & $0.0033(11)$ \\
\hline C10 & $0.0435(12)$ & $0.0402(11)$ & $0.0460(12)$ & $0.0014(11)$ & $-0.0031(11)$ & $0.0038(10)$ \\
\hline C11 & $0.0390(13)$ & $0.0386(11)$ & $0.0472(13)$ & $-0.0011(10)$ & $-0.0030(11)$ & $0.0015(10)$ \\
\hline C12 & $0.061(2)$ & $0.0425(12)$ & $0.0514(14)$ & $-0.0048(12)$ & $0.0007(13)$ & $0.0055(12)$ \\
\hline C13 & $0.076(2)$ & $0.076(2)$ & $0.085(2)$ & $-0.032(2)$ & $0.006(2)$ & $-0.019(2)$ \\
\hline C14 & $0.104(3)$ & $0.069(2)$ & $0.066(2)$ & $-0.015(2)$ & $0.004(2)$ & $0.030(2)$ \\
\hline C15 & $0.081(2)$ & $0.065(2)$ & $0.056(2)$ & $0.0034(14)$ & $0.013(2)$ & $-0.017(2)$ \\
\hline C16 & $0.0497(14)$ & $0.0339(11)$ & $0.0488(13)$ & $-0.0030(10)$ & $-0.0024(11)$ & $-0.0023(10)$ \\
\hline C17 & $0.086(2)$ & $0.0396(14)$ & $0.068(2)$ & $0.0104(14)$ & $-0.005(2)$ & $0.007(2)$ \\
\hline 01 & $0.0416(8)$ & $0.0451(9)$ & $0.0476(8)$ & $-0.0044(8)$ & $-0.0047(7)$ & $-0.0015(7)$ \\
\hline $\mathrm{O} 2$ & $0.0484(10)$ & $0.0440(9)$ & $0.0689(11)$ & $0.0002(9)$ & $0.0026(9)$ & $0.0079(8)$ \\
\hline $\mathrm{O} 3$ & $0.0636(11)$ & $0.0420(9)$ & $0.0546(10)$ & $0.0078(8)$ & $0.0032(9)$ & $0.0043(8)$ \\
\hline $\mathrm{O} 4$ & $0.0401(9)$ & $0.0520(10)$ & $0.0562(10)$ & $0.0032(8)$ & $0.0064(7)$ & $0.0023(8)$ \\
\hline
\end{tabular}

Table S4. Bond Distances in Compound 6, $\AA$

\begin{tabular}{|llllll|}
\hline $\mathrm{C} 1-\mathrm{C} 11$ & $1.528(3)$ & $\mathrm{C} 1-\mathrm{C} 2$ & $1.534(4)$ & $\mathrm{C} 1-\mathrm{C} 12$ & $1.554(3)$ \\
$\mathrm{C} 1-\mathrm{H} 1$ & $1.04(3)$ & $\mathrm{C} 2-\mathrm{C} 3$ & $1.488(4)$ & $\mathrm{C} 2-\mathrm{H} 2 \mathrm{~b}$ & $1.05(3)$ \\
$\mathrm{C} 2-\mathrm{H} 2 \mathrm{a}$ & $1.07(3)$ & $\mathrm{C} 3-\mathrm{C} 4$ & $1.325(4)$ & $\mathrm{C} 3-\mathrm{H} 3$ & $1.01(3)$ \\
$\mathrm{C} 4-\mathrm{C} 15$ & $1.503(4)$ & $\mathrm{C} 4-\mathrm{C} 5$ & $1.514(3)$ & $\mathrm{C} 5-\mathrm{C} 6$ & $1.551(3)$ \\
$\mathrm{C} 5-\mathrm{C} 11$ & $1.548(3)$ & $\mathrm{C} 5-\mathrm{H} 5$ & $1.02(3)$ & $\mathrm{C} 6-\mathrm{O} 1$ & $1.430(3)$ \\
$\mathrm{C} 6-\mathrm{C} 7$ & $1.519(3)$ & $\mathrm{C}-\mathrm{H} 6$ & $0.99(2)$ & $\mathrm{C}-\mathrm{C} 8$ & $1.355(3)$ \\
$\mathrm{C} 7-\mathrm{C} 16$ & $1.444(3)$ & $\mathrm{C} 8-\mathrm{O} 4$ & $1.345(3)$ & $\mathrm{C} 8-\mathrm{C} 9$ & $1.490(3)$ \\
$\mathrm{C} 9-\mathrm{C} 10$ & $1.518(3)$ & $\mathrm{C} 9-\mathrm{H} 9 \mathrm{~b}$ & $0.92(3)$ & $\mathrm{C} 9-\mathrm{H} 9 \mathrm{a}$ & $1.03(3)$ \\
$\mathrm{C} 10-\mathrm{O} 1$ & $1.441(3)$ & $\mathrm{C} 10-\mathrm{C} 11$ & $1.548(3)$ & $\mathrm{C} 10-\mathrm{H} 10$ & $0.98(3)$ \\
$\mathrm{C} 11-\mathrm{H} 11$ & $0.94(2)$ & $\mathrm{C} 12-\mathrm{C} 13$ & $1.519(5)$ & $\mathrm{C} 12-\mathrm{C} 14$ & $1.524(4)$ \\
$\mathrm{C} 12-\mathrm{H} 12$ & $0.99(3)$ & $\mathrm{C} 13-\mathrm{H} 13 \mathrm{a}$ & $1.11(6)$ & $\mathrm{C} 13-\mathrm{H} 13 \mathrm{~b}$ & $0.91(3)$ \\
$\mathrm{C} 13-\mathrm{H} 13 \mathrm{c}$ & $1.07(4)$ & $\mathrm{C} 14-\mathrm{H} 14 \mathrm{c}$ & $1.04(4)$ & $\mathrm{C} 14-\mathrm{H} 14 \mathrm{a}$ & $1.01(5)$ \\
$\mathrm{C} 14-\mathrm{H} 14 \mathrm{~b}$ & $1.03(5)$ & $\mathrm{C} 15-\mathrm{H} 15 \mathrm{~b}$ & $0.99(4)$ & $\mathrm{C} 15-\mathrm{H} 15 \mathrm{c}$ & $1.05(4)$ \\
$\mathrm{C} 15-\mathrm{H} 15 \mathrm{a}$ & $0.99(3)$ & $\mathrm{C} 16-\mathrm{O} 2$ & $1.224(3)$ & $\mathrm{C} 16-\mathrm{O} 3$ & $1.348(3)$ \\
$\mathrm{C} 17-\mathrm{O} 3$ & $1.444(3)$ & $\mathrm{C} 17-\mathrm{H} 17 \mathrm{c}$ & $1.00(4)$ & $\mathrm{C} 17-\mathrm{H} 17 \mathrm{a}$ & $0.98(5)$ \\
$\mathrm{C} 17-\mathrm{H} 17 \mathrm{~b}$ & $1.00(4)$ & $\mathrm{O} 4-\mathrm{H} 4$ & $1.07(4)$ & & \\
\hline
\end{tabular}


Table S5. Bond Angles in Compound 6,

\begin{tabular}{|c|c|c|c|c|c|}
\hline C11-C1-C2 & 108.7(2) & C11-C1-C12 & $113.6(2)$ & C2-C1-C12 & $111.9(2)$ \\
\hline $\mathrm{C} 11-\mathrm{C} 1-\mathrm{H} 1$ & $107(2)$ & C2-C1-H1 & $110(2)$ & $\mathrm{C} 12-\mathrm{C} 1-\mathrm{H} 1$ & $105(2)$ \\
\hline C3-C2-C1 & $110.5(2)$ & $\mathrm{C} 3-\mathrm{C} 2-\mathrm{H} 2 \mathrm{~b}$ & $108(2)$ & $\mathrm{C} 1-\mathrm{C} 2-\mathrm{H} 2 \mathrm{~b}$ & $112(2)$ \\
\hline $\mathrm{C} 3-\mathrm{C} 2-\mathrm{H} 2 \mathrm{a}$ & $111(2)$ & $\mathrm{C} 1-\mathrm{C} 2-\mathrm{H} 2 \mathrm{a}$ & $110(2)$ & $\mathrm{H} 2 \mathrm{~b}-\mathrm{C} 2-\mathrm{H} 2 \mathrm{a}$ & 105(3) \\
\hline C4-C3-C2 & $124.0(2)$ & C4-C3-H3 & $115(2)$ & C2-C3-H3 & $121(2)$ \\
\hline C3-C4-C15 & $122.9(3)$ & C3-C4-C5 & 121.7(2) & C15-C4-C5 & $115.4(3)$ \\
\hline C4-C5-C6 & $110.3(2)$ & C4-C5-C11 & $114.4(2)$ & C6-C5-C11 & $100.3(2)$ \\
\hline C4-C5-H5 & $110.9(14)$ & C6-C5-H5 & $106.2(14)$ & C11-C5-H5 & $113.9(14)$ \\
\hline O1-C6-C7 & $109.2(2)$ & O1-C6-C5 & $102.4(2)$ & C7-C6-C5 & $112.3(2)$ \\
\hline O1-C6-H6 & $111(2)$ & C7-C6-H6 & $106(2)$ & C5-C6-H6 & $116.4(14)$ \\
\hline C8-C7-C16 & $120.1(2)$ & C8-C7-C6 & 118.1(2) & C16-C7-C6 & $121.7(2)$ \\
\hline O4-C8-C7 & $124.8(2)$ & O4-C8-C9 & $113.3(2)$ & C7-C8-C9 & $121.9(2)$ \\
\hline C8-C9-C10 & 109.6(2) & C8-C9-H9b & 107(2) & $\mathrm{C} 10-\mathrm{C} 9-\mathrm{H} 9 \mathrm{~b}$ & 112(2) \\
\hline C8-C9-H9a & 109.1(13) & C10-C9-H9a & $111.6(13)$ & $\mathrm{H} 9 \mathrm{~b}-\mathrm{C} 9-\mathrm{H} 9 \mathrm{a}$ & $108(2)$ \\
\hline O1-C10-C9 & $107.4(2)$ & O1-C10-C11 & $105.6(2)$ & C9-C10-C11 & $112.5(2)$ \\
\hline O1-C10-H10 & $106(2)$ & C9-C10-H10 & $110(2)$ & C11-C10-H10 & $115(2)$ \\
\hline C1-C11-C5 & $114.4(2)$ & C1-C11-C10 & $113.1(2)$ & C5-C11-C10 & $103.6(2)$ \\
\hline C1-C11-H11 & 108.2(13) & $\mathrm{C} 5-\mathrm{C} 11-\mathrm{H} 11$ & 109.1(13) & $\mathrm{C} 10-\mathrm{C} 11-\mathrm{H} 11$ & $108.3(13)$ \\
\hline C13-C12-C14 & 110.1(3) & C13-C12-C1 & $112.0(2)$ & C14-C12-C1 & $111.0(2)$ \\
\hline C13-C12-H12 & $109(2)$ & C14-C12-H12 & $109(2)$ & $\mathrm{C} 1-\mathrm{C} 12-\mathrm{H} 12$ & $106(2)$ \\
\hline $\mathrm{C} 12-\mathrm{C} 13-\mathrm{H} 13 \mathrm{a}$ & 112(3) & C12-C13-H13b & $105(2)$ & $\mathrm{H} 13 \mathrm{a}-\mathrm{C} 13-\mathrm{H} 13 \mathrm{~b}$ & $114(4)$ \\
\hline $\mathrm{C} 12-\mathrm{C} 13-\mathrm{H} 13 \mathrm{C}$ & $111(2)$ & H13a-C13-H13c & $109(4)$ & $\mathrm{H} 13 \mathrm{~b}-\mathrm{C} 13-\mathrm{H} 13 \mathrm{c}$ & 106(3) \\
\hline C12-C14-H14c & $112(2)$ & C12-C14-H14a & $108(3)$ & $\mathrm{H} 14 \mathrm{C}-\mathrm{C} 14-\mathrm{H} 14 \mathrm{a}$ & 107(3) \\
\hline C12-C14-H14b & 108(3) & $\mathrm{H} 14 \mathrm{C}-\mathrm{C} 14-\mathrm{H} 14 \mathrm{~b}$ & 106(3) & $\mathrm{H} 14 \mathrm{a}-\mathrm{C} 14-\mathrm{H} 14 \mathrm{~b}$ & $115(4)$ \\
\hline C4-C15-H15b & $113(2)$ & C4-C15-H15c & $111(2)$ & $\mathrm{H} 15 \mathrm{~b}-\mathrm{C} 15-\mathrm{H} 15 \mathrm{c}$ & 109(3) \\
\hline C4-C15-H15a & $114(2)$ & $\mathrm{H} 15 \mathrm{~b}-\mathrm{C} 15-\mathrm{H} 15 \mathrm{a}$ & $103(3)$ & H15C-C15-H15a & $106(2)$ \\
\hline O2-C16-O3 & $122.0(2)$ & O2-C16-C7 & $125.5(2)$ & O3-C16-C7 & $112.4(2)$ \\
\hline O3-C17-H17c & 112(2) & O3-C17-H17a & $108(2)$ & $\mathrm{H} 17 \mathrm{C}-\mathrm{C} 17-\mathrm{H} 17 \mathrm{a}$ & 108(3) \\
\hline O3-C17-H17b & $102(2)$ & $\mathrm{H} 17 \mathrm{C}-\mathrm{C} 17-\mathrm{H} 17 \mathrm{~b}$ & 116(3) & $\mathrm{H} 17 \mathrm{a}-\mathrm{C} 17-\mathrm{H} 17 \mathrm{~b}$ & 110(3) \\
\hline C6-O1-C10 & $102.2(2)$ & C16-O3-C17 & $115.8(2)$ & C8-O4-H4 & $106(2)$ \\
\hline
\end{tabular}




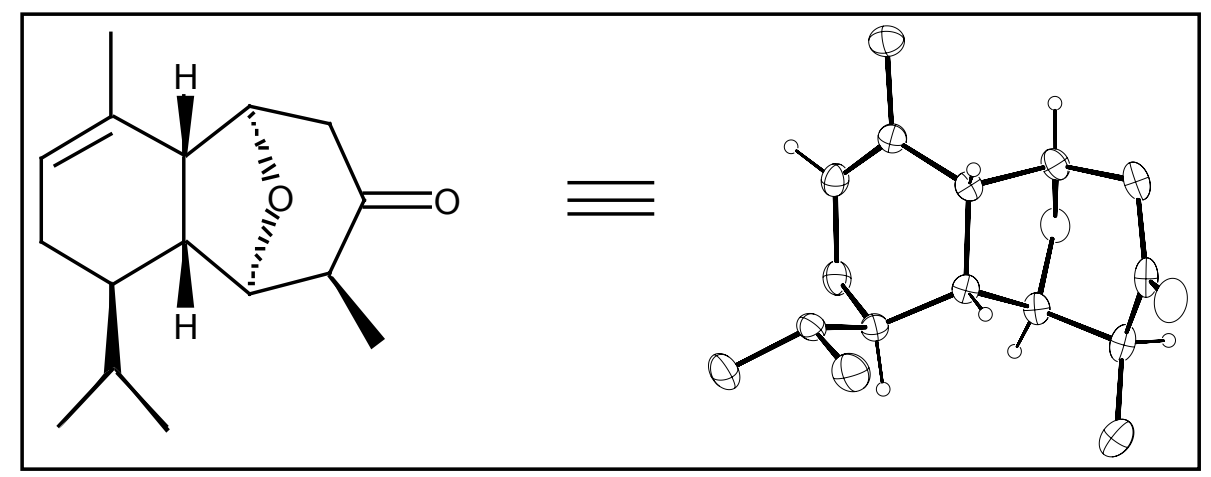

\section{$X$-ray Structure Determination of Compound 8}

Compound 8, $\mathrm{C}_{16} \mathrm{H}_{24} \mathrm{O}_{2}$, crystallizes in the orthorhombic space group $\mathrm{P} 2{ }_{1} 2_{1} 2_{1}$ (systematic absences $\mathrm{h} 00: \mathrm{h}=$ odd, 0k0: $\mathrm{k}=$ odd, and 001: $\mathrm{l}=$ odd) with $\mathrm{a}=5.6107(8) \AA, \mathrm{b}=9.725(2) \AA$, $\mathrm{c}=25.856(4) \AA, \mathrm{V}=1410.8(4) \AA^{3}, \mathrm{Z}=4$ and $\mathrm{d}_{\text {calc }}=1.169 \mathrm{~g} / \mathrm{cm}^{3}$. X-ray intensity data were collected on a Rigaku Mercury CCD area detector employing graphite-monochromated $\mathrm{Mo}_{\mathrm{\alpha}} \mathrm{K}_{\alpha}$ radiation $(\lambda=0.71069 \AA)$ at a temperature of $143^{\circ} \mathrm{K}$. Indexing was performed from a series of four $0.5^{\circ}$ oscillation images with exposures of 30 seconds per frame. A hemisphere of data was collected using 80 second exposures and a crystal-to-detector distance of $40 \mathrm{~mm}$. A total of 440 images were collected: one sweep was performed using $\phi$-scans from $-90^{\circ}$ to $+90^{\circ}$ in $0.5^{\circ}$ steps at $\omega=0^{\circ}$ and $\chi=0^{\circ}$ with a detector swing angle of $-10^{\circ}$; a second sweep was done using $\omega$-scans from $20^{\circ}$ to $+20^{\circ}$ in $0.5^{\circ}$ steps at $\chi=-90^{\circ}$ and $\phi=0^{\circ}$ with a detector swing angle of $-10^{\circ}$. Oscillation

images were processed using CrystalClear ${ }^{11}$, producing a listing of unaveraged $\mathrm{F}^{2}$ and $\sigma\left(\mathrm{F}^{2}\right)$ values which were then passed to the CrystalStructure ${ }^{12}$ program package for further processing and structure solution on a Dell Pentium III computer. A total of 7530 reflections were measured over the ranges $5.24 \leq 2 \theta \leq 50.64^{\circ},-5 \leq \mathrm{h} \leq 6,-11 \leq \mathrm{k} \leq 11,-30 \leq 1 \leq 19$ yielding 2473 unique reflections $\left(R_{\text {int }}=0.0264\right)$. The intensity data were corrected for Lorentz and polarization effects and for absorption. using REQAB ${ }^{13}$ (minimum and maximum transmission $0.830,1.000$ ).

The structure was solved by direct methods $\left(\operatorname{SIR} 97^{14}\right)$. Refinement was by full-matrix least squares based on $\mathrm{F}^{2}$ using SHELXL-97 ${ }^{15}$. All reflections were used during refinement 
$\left(\mathrm{F}^{2}\right.$ 's that were experimentally negative were replaced by $\left.\mathrm{F}^{2}=0\right)$. The weighting scheme used was $\mathrm{w}=1 /\left[\sigma^{2}\left(\mathrm{~F}_{\mathrm{o}}^{2}\right)+0.0410 \mathrm{P}^{2}+0.2173 \mathrm{P}\right]$ where $\mathrm{P}=\left(\mathrm{F}_{\mathrm{o}}^{2}+2 \mathrm{~F}_{\mathrm{c}}^{2}\right) / 3$. Non-hydrogen atoms were refined anisotropically and hydrogen atoms were refined using a "riding" model. Refinement converged to $\mathrm{R}_{1}=0.0440$ and $\mathrm{wR}_{2}=0.0925$ for 2192 reflections for which $\mathrm{F}>4 \sigma(\mathrm{F})$ and $\mathrm{R}_{1}=0.0525, \mathrm{wR}_{2}=0.0972$ and $\mathrm{GOF}=1.109$ for all 2473 unique, non-zero reflections and 167 variables $^{16}$. The maximum $\Delta / \sigma$ in the final cycle of least squares was 0.000 and the two most prominent peaks in the final difference Fourier were +0.122 and $-0.158 \mathrm{e} / \AA^{3}$.

Table S6. lists cell information, data collection parameters, and refinement data. Final positional and equivalent isotropic thermal parameters are given in Table S7. Anisotropic thermal parameters are in Table S8. Tables S9 and S10 list bond distances and bond angles. Figure 1. is an ORTEP $^{17}$ representation of the molecule with $30 \%$ probability thermal ellipsoids displayed.

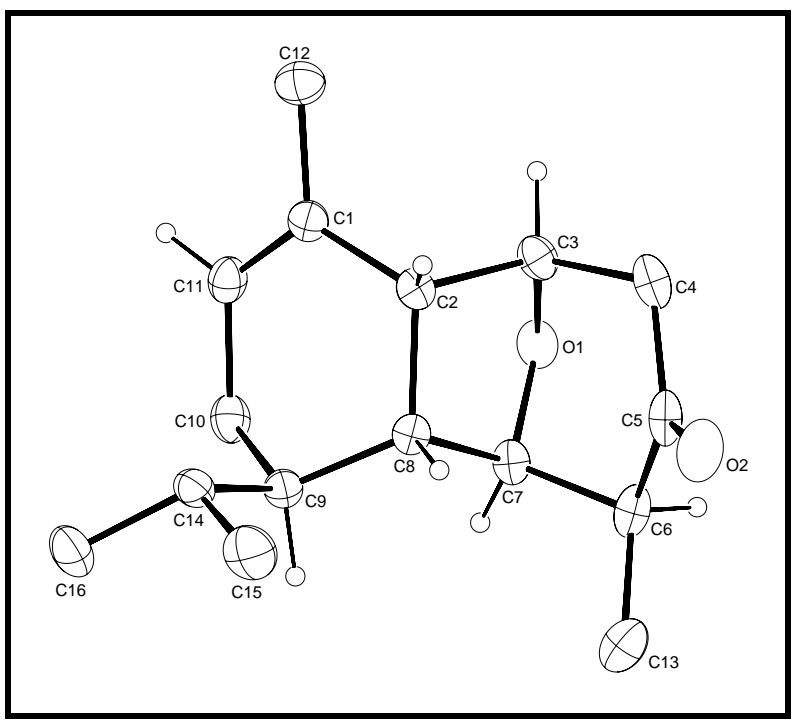

Figure S2. ORTEP drawing of the title compound with $30 \%$ probability thermal ellipsoids. 


\section{Table S6. Summary of Structure Determination of Compound 8}

Formula:

Formula weight:

Crystal class:

Space group:

Z

Cell constants:

$$
\begin{aligned}
& a \\
& b \\
& c \\
& \text { V }
\end{aligned}
$$$$
\mathrm{c}
$$

$\mu$

crystal size, $\mathrm{mm}$

$\mathrm{D}_{\text {calc }}$

$\mathrm{F}(000)$

Radiation:

$2 \theta$ range

hkl collected:

No. reflections measured:

No. unique reflections:

No. observed reflections

No. reflections used in refinement

No. parameters

$R$ indices $(F>4 \sigma)$

$\mathrm{R}$ indices (all data)

GOF:

Final Difference Peaks, e/ $\AA^{3}$
$\mathrm{C}_{16} \mathrm{H}_{24} \mathrm{O}_{2}$

248.35

orthorhombic

$\mathrm{P} 2{ }_{1} 22_{1}$ (\#19)

4

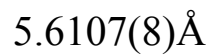

$9.725(2) \AA$

$25.856(4) \AA$

$1410.8(4) \AA^{3}$

$0.75 \mathrm{~cm}^{-1}$

$0.45 \times 0.05 \times 0.03$

$1.169 \mathrm{~g} / \mathrm{cm}^{3}$

544

$\mathrm{Mo}-\mathrm{K}_{\alpha}(\lambda=0.71070 \AA)$

$5.24-50.64^{\circ}$

$-5 \leq \mathrm{h} \leq 6 ;-11 \leq \mathrm{k} \leq 11 ;-30 \leq 1 \leq 19$

7530

$2473\left(\mathrm{R}_{\mathrm{int}}=0.0264\right)$

$2192(\mathrm{~F}>4 \sigma)$

2473

167

$\mathrm{R}_{1}=0.0440$

$\mathrm{wR}_{2}=0.0925$

$\mathrm{R}_{1}=0.0525$

$\mathrm{wR}_{2}=0.0972$

1.109

$+0.122,-0.15$ 
Table S7. Refined Positional Parameters for Compound 8

\begin{tabular}{|c|c|c|c|c|}
\hline Atom & $\mathrm{X}$ & $\mathrm{y}$ & Z & $\mathrm{U}_{\mathrm{eq}}, \AA^{2}$ \\
\hline C1 & $0.8303(3)$ & $0.8091(2)$ & $0.61493(7)$ & $0.0320(4)$ \\
\hline $\mathrm{C} 2$ & $0.8786(3)$ & $0.6633(2)$ & $0.59781(7)$ & $0.0298(4)$ \\
\hline $\mathrm{H} 2$ & 1.0501 & 0.6446 & 0.5995 & 0.040 \\
\hline C3 & $0.7884(3)$ & $0.6390(2)$ & $0.54228(7)$ & $0.0346(5)$ \\
\hline $\mathrm{H} 3$ & 0.8065 & 0.7225 & 0.5214 & 0.046 \\
\hline C4 & $0.9142(4)$ & $0.5161(2)$ & $0.51609(8)$ & $0.0414(5)$ \\
\hline $\mathrm{H} 4 \mathrm{a}$ & 0.8615 & 0.5096 & 0.4804 & 0.055 \\
\hline $\mathrm{H} 4 \mathrm{~b}$ & 1.0848 & 0.5321 & 0.5159 & 0.055 \\
\hline C5 & $0.8629(4)$ & $0.3822(2)$ & $0.54334(7)$ & $0.0389(5)$ \\
\hline C6 & $0.6124(4)$ & $0.3653(2)$ & $0.56395(8)$ & $0.0389(5)$ \\
\hline $\mathrm{H} 6$ & 0.5062 & 0.3526 & 0.5342 & 0.052 \\
\hline $\mathrm{C} 7$ & $0.5475(3)$ & $0.5036(2)$ & $0.58957(7)$ & $0.0320(4)$ \\
\hline $\mathrm{H} 7$ & 0.3922 & 0.4969 & 0.6067 & 0.043 \\
\hline C8 & $0.7400(3)$ & $0.5508(2)$ & $0.62842(7)$ & $0.0291(5)$ \\
\hline $\mathrm{H} 8$ & 0.8486 & 0.4734 & 0.6343 & 0.039 \\
\hline C9 & $0.6431(3)$ & $0.5972(2)$ & $0.68107(7)$ & $0.0323(5)$ \\
\hline H9 & 0.5296 & 0.5272 & 0.6927 & 0.043 \\
\hline C10 & $0.5025(4)$ & $0.7315(2)$ & $0.67330(8)$ & $0.0375(5)$ \\
\hline $\mathrm{H} 10 \mathrm{a}$ & 0.3637 & 0.7138 & 0.6520 & 0.050 \\
\hline $\mathrm{H} 10 \mathrm{~b}$ & 0.4480 & 0.7655 & 0.7065 & 0.050 \\
\hline C11 & $0.6567(4)$ & $0.8385(2)$ & $0.64776(7)$ & $0.0363(5)$ \\
\hline $\mathrm{H} 11$ & 0.6283 & 0.9304 & 0.6555 & 0.048 \\
\hline C12 & $0.9899(4)$ & $0.9168(2)$ & $0.59186(8)$ & $0.0439(5)$ \\
\hline $\mathrm{H} 12 \mathrm{a}$ & 0.9533 & 1.0047 & 0.6068 & 0.066 \\
\hline $\mathrm{H} 12 \mathrm{~b}$ & 0.9652 & 0.9206 & 0.5551 & 0.066 \\
\hline $\mathrm{H} 12 \mathrm{c}$ & 1.1532 & 0.8940 & 0.5989 & 0.066 \\
\hline C13 & $0.5797(4)$ & $0.2429(2)$ & $0.59939(9)$ & $0.0513(6)$ \\
\hline $\mathrm{H} 13 \mathrm{a}$ & 0.6247 & 0.1604 & 0.5815 & 0.077 \\
\hline $\mathrm{H} 13 \mathrm{~b}$ & 0.4156 & 0.2367 & 0.6097 & 0.077 \\
\hline $\mathrm{H} 13 \mathrm{c}$ & 0.6780 & 0.2539 & 0.6295 & 0.077 \\
\hline C14 & $0.8369(4)$ & $0.6098(2)$ & $0.72320(7)$ & $0.0365(5)$ \\
\hline $\mathrm{H} 14$ & 0.9458 & 0.6840 & 0.7132 & 0.049 \\
\hline C15 & $0.9822(5)$ & $0.4782(2)$ & $0.72891(9)$ & $0.0557(6)$ \\
\hline $\mathrm{H} 15 \mathrm{a}$ & 1.0856 & 0.4860 & 0.7583 & 0.084 \\
\hline $\mathrm{H} 15 \mathrm{~b}$ & 1.0760 & 0.4640 & 0.6983 & 0.084 \\
\hline $\mathrm{H} 15 \mathrm{c}$ & 0.8765 & 0.4016 & 0.7337 & 0.084 \\
\hline C16 & $0.7278(5)$ & $0.6469(3)$ & $0.77552(8)$ & $0.0580(7)$ \\
\hline $\mathrm{H} 16 \mathrm{a}$ & 0.6151 & 0.5774 & 0.7854 & 0.087 \\
\hline $\mathrm{H} 16 \mathrm{~b}$ & 0.6483 & 0.7340 & 0.7730 & 0.087 \\
\hline $\mathrm{H} 16 \mathrm{c}$ & 0.8516 & 0.6525 & 0.8011 & 0.087 \\
\hline $\mathrm{O} 1$ & $0.5412(2)$ & $0.60755(13)$ & $0.54995(5)$ & $0.0359(3)$ \\
\hline $\mathrm{O} 2$ & $1.0166(3)$ & $0.2940(2)$ & $0.54870(6)$ & $0.0522(4)$ \\
\hline
\end{tabular}

Table S8. Refined Thermal Parameters (U's) for Compound 8 


\begin{tabular}{|c|c|c|c|c|c|c|}
\hline Atom & $\mathrm{U}_{11}$ & $\mathrm{U}_{22}$ & $\mathrm{U}_{33}$ & $\mathrm{U}_{23}$ & $\mathrm{U}_{13}$ & $\mathrm{U}_{12}$ \\
\hline C1 & $0.0329(11)$ & $0.0318(10)$ & $0.0314(10)$ & $0.0021(9)$ & $-0.0038(9)$ & $0.0032(8)$ \\
\hline $\mathrm{C} 2$ & $0.0250(10)$ & $0.0344(10)$ & $0.0299(10)$ & $0.0001(9)$ & $0.0000(8)$ & $0.0045(8)$ \\
\hline C3 & $0.0330(12)$ & $0.0416(12)$ & $0.0291(10)$ & $0.0008(9)$ & $-0.0012(8)$ & $0.0055(8)$ \\
\hline C4 & $0.0395(12)$ & $0.0563(14)$ & $0.0284(11)$ & $-0.0074(10)$ & $-0.0003(8)$ & $0.0110(10)$ \\
\hline C5 & $0.0393(12)$ & $0.0443(12)$ & $0.0330(11)$ & $-0.0153(10)$ & $-0.0078(9)$ & $0.0117(10)$ \\
\hline C6 & $0.0390(12)$ & $0.0365(12)$ & $0.0412(12)$ & $-0.0130(10)$ & $-0.0103(9)$ & $0.0061(9)$ \\
\hline $\mathrm{C} 7$ & $0.0303(10)$ & $0.0357(11)$ & $0.0300(10)$ & $-0.0036(9)$ & $-0.0028(8)$ & $0.0051(8)$ \\
\hline C8 & $0.0264(10)$ & $0.0298(10)$ & $0.0311(11)$ & $-0.0004(8)$ & $-0.0031(8)$ & $0.0036(8)$ \\
\hline C9 & $0.0320(11)$ & $0.0336(11)$ & $0.0311(11)$ & $-0.0012(9)$ & $0.0016(8)$ & $-0.0043(9)$ \\
\hline C10 & $0.0331(11)$ & $0.0417(12)$ & $0.0378(12)$ & $-0.0075(9)$ & $0.0030(9)$ & $0.0054(9)$ \\
\hline C11 & $0.0411(12)$ & $0.0319(10)$ & $0.0360(11)$ & $-0.0037(9)$ & $-0.0035(9)$ & $0.0072(9)$ \\
\hline C12 & $0.0455(13)$ & $0.0402(12)$ & $0.0461(12)$ & $0.0083(10)$ & $-0.0029(10)$ & $0.0005(10)$ \\
\hline C13 & $0.054(2)$ & $0.0374(12)$ & $0.062(2)$ & $-0.0063(12)$ & $-0.0097(12)$ & $0.0017(10)$ \\
\hline C14 & $0.0409(12)$ & $0.0411(11)$ & $0.0277(10)$ & $0.0036(9)$ & $-0.0016(9)$ & $-0.0091(10)$ \\
\hline C15 & $0.059(2)$ & $0.064(2)$ & $0.0442(13)$ & $0.0096(12)$ & $-0.0165(12)$ & $0.0018(13)$ \\
\hline C16 & $0.073(2)$ & $0.072(2)$ & $0.0290(12)$ & $-0.0026(12)$ & $0.0029(11)$ & $-0.0145(13)$ \\
\hline O1 & $0.0318(8)$ & $0.0418(8)$ & $0.0341(7)$ & $-0.0021(6)$ & $-0.0057(5)$ & $0.0076(6)$ \\
\hline $\mathrm{O} 2$ & $0.0443(9)$ & $0.0519(9)$ & $0.0605(10)$ & $-0.0144(8)$ & $-0.0067(7)$ & $0.0192(8)$ \\
\hline
\end{tabular}

Table S9. Bond Distances in Compound 8, $\AA$

\begin{tabular}{|llllll|}
\hline C1-C11 & $1.323(3)$ & C1-C12 & $1.502(3)$ & C1-C2 & $1.510(3)$ \\
C2-C3 & $1.541(3)$ & C2-C8 & $1.558(3)$ & C3-O1 & $1.434(2)$ \\
C3-C4 & $1.544(3)$ & C4-C5 & $1.509(3)$ & C5-O2 & $1.224(2)$ \\
C5-C6 & $1.512(3)$ & C6-C13 & $1.513(3)$ & C6-C7 & $1.543(3)$ \\
C7-O1 & $1.440(2)$ & C7-C8 & $1.545(3)$ & C8-C9 & $1.534(3)$ \\
C9-C10 & $1.539(3)$ & C9-C14 & $1.544(3)$ & C10-C11 & $1.505(3)$ \\
C14-C15 & $1.525(3)$ & C14-C16 & $1.528(3)$ & & \\
\hline
\end{tabular}

Table S10. Bond Angles in Compound 8,

\begin{tabular}{|llllll|}
\hline C11-C1-C12 & $122.9(2)$ & C11-C1-C2 & $121.5(2)$ & C12-C1-C2 & $115.6(2)$ \\
C1-C2-C3 & $111.0(2)$ & C1-C2-C8 & $114.9(2)$ & C3-C2-C8 & $101.64(14)$ \\
O1-C3-C2 & $102.8(2)$ & O1-C3-C4 & $109.7(2)$ & C2-C3-C4 & $112.2(2)$ \\
C5-C4-C3 & $112.1(2)$ & O2-C5-C4 & $121.6(2)$ & O2-C5-C6 & $122.6(2)$ \\
C4-C5-C6 & $115.8(2)$ & C13-C6-C5 & $114.3(2)$ & C13-C6-C7 & $113.4(2)$ \\
C5-C6-C7 & $106.0(2)$ & O1-C7-C8 & $105.7(2)$ & O1-C7-C6 & $108.20(14)$ \\
C8-C7-C6 & $111.9(2)$ & C9-C8-C7 & $114.6(2)$ & C9-C8-C2 & $114.9(2)$ \\
C7-C8-C2 & $103.14(14)$ & C8-C9-C10 & $108.4(2)$ & C8-C9-C14 & $113.6(2)$ \\
C10-C9-C14 & $112.7(2)$ & C11-C10-C9 & $110.4(2)$ & C1-C11-C10 & $123.7(2)$ \\
C15-C14-C16 & $109.1(2)$ & C15-C14-C9 & $112.2(2)$ & C16-C14-C9 & $111.2(2)$ \\
C3-O1-C7 & $102.96(14)$ & & & & \\
\hline
\end{tabular}




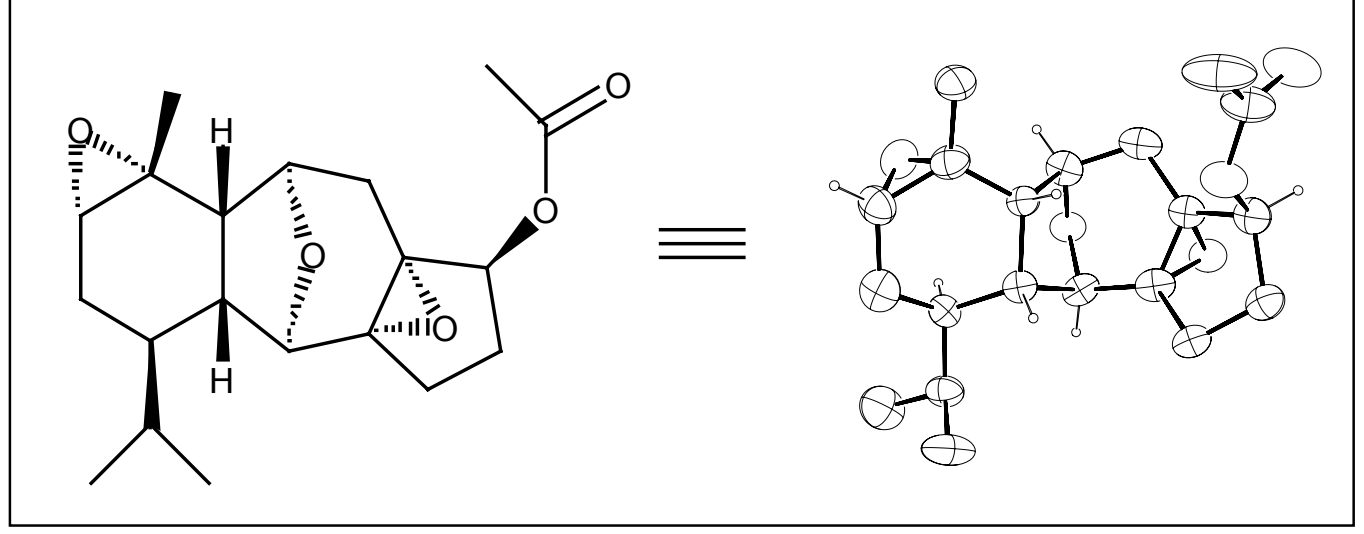

X-ray Structure Determination of Compound 18

Compound 18, $\mathrm{C}_{20} \mathrm{H}_{28} \mathrm{O}_{6}$, crystallizes in the triclinic space group P1 with a=13.906(9) $\AA$, $b=5.635(3) \AA, c=14.432(9) \AA, \alpha=90.22(3)^{\circ}, \beta=104.70(2)^{\circ}, \gamma=90.45(3)^{\circ}, V=1093.8(12) \AA^{3}, Z=2$ and $\mathrm{d}_{\text {calc }}=1.107 \mathrm{~g} / \mathrm{cm}^{3}$. X-ray intensity data were collected on a Rigaku Mercury CCD area detector employing graphite-monochromated Mo- $\mathrm{K}_{\alpha}$ radiation $(\lambda=0.71069 \AA)$ at a temperature of $143^{\circ} \mathrm{K}$. Indexing was performed from a series of four $0.5^{\circ}$ oscillation images with exposures of 30 seconds per frame. A hemisphere of data was collected using 100 second exposures and a crystal-to-detector distance of $40 \mathrm{~mm}$. A total of 440 images were collected: one sweep was performed using $\phi$-scans from $-90^{\circ}$ to $+90^{\circ}$ in $0.5^{\circ}$ steps at $\omega=0^{\circ}$ and $\chi=0^{\circ}$ with a detector swing angle of $-10^{\circ}$; a second sweep was done using $\omega$-scans from $-20^{\circ}$ to $+20^{\circ}$ in $0.5^{\circ}$ steps at $\chi$ $=-90^{\circ}$ and $\phi=0^{\circ}$ with a detector swing angle of $-10^{\circ}$. Oscillation images were processed using CrystalClear ${ }^{18}$, producing a listing of unaveraged $\mathrm{F}^{2}$ and $\sigma\left(\mathrm{F}^{2}\right)$ values which were then passed to the CrystalStructure ${ }^{19}$ program package for further processing and structure solution on a Dell Pentium III computer. A total of 4622 reflections were measured over the ranges 5.84 $\leq 2 \theta \leq 44.92 \circ,-14 \leq \mathrm{h} \leq 14,-5 \leq \mathrm{k} \leq 5,-15 \leq 1 \leq 11$ yielding 3796 unique reflections $\left(\mathrm{R}_{\text {int }}=\right.$ 0.0413). The intensity data were corrected for Lorentz and polarization effects and for absorption. using REQAB ${ }^{20}$ (minimum and maximum transmission 0.534, 1.000).

The structure was solved by direct methods $\left(\operatorname{SIR} 97^{21}\right)$. Refinement was by full-matrix least squares based on $\mathrm{F}^{2}$ using SHELXL-97 ${ }^{22}$. All reflections were used during refinement 
$\left(\mathrm{F}^{2}\right.$ 's that were experimentally negative were replaced by $\mathrm{F}^{2}=0$ ). The weighting scheme used was $\mathrm{w}=1 /\left[\sigma^{2}\left(\mathrm{~F}_{\mathrm{o}}^{2}\right)+0.2000 \mathrm{P}^{2}+0.0000 \mathrm{P}\right]$ where $\mathrm{P}=\left(\mathrm{F}_{\mathrm{o}}^{2}+2 \mathrm{~F}_{\mathrm{c}}^{2}\right) / 3$. Non-hydrogen atoms were refined anisotropically and hydrogen atoms were refined using a "riding" model. Refinement converged to $\mathrm{R}_{1}=0.1252$ and $\mathrm{wR}_{2}=0.3121$ for 2746 reflections for which $\mathrm{F}>4 \sigma(\mathrm{F})$ and $\mathrm{R}_{1}=0.1471, \mathrm{wR}_{2}=0.3409$ and $\mathrm{GOF}=1.322$ for all 3796 unique, non-zero reflections and 452 variables $^{23}$. The maximum $\Delta / \sigma$ in the final cycle of least squares was 0.000 and the two most prominent peaks in the final difference Fourier were +0.943 and $-0.558 \mathrm{e} / \AA^{3}$.

Table S11. lists cell information, data collection parameters, and refinement data. Final positional and equivalent isotropic thermal parameters are given in Table S12. Anisotropic thermal parameters are in Table S13. Tables S14 and S15 list bond distances and bond angles. Figures 1. and 2. Are ORTEP ${ }^{24}$ representations of the molecule with $30 \%$ probability thermal ellipsoids displayed.

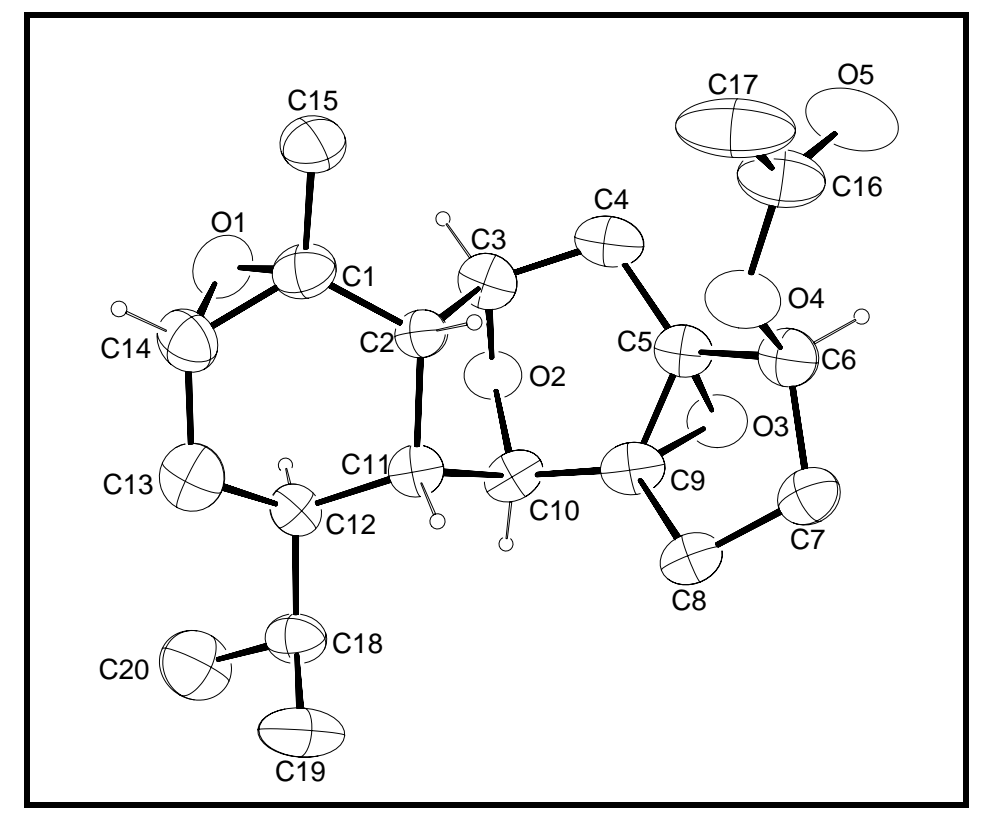

Figure S3. ORTEP drawing of molecule no. 1 of the asymmetric unit with $30 \%$ probability thermal ellipsoids. 


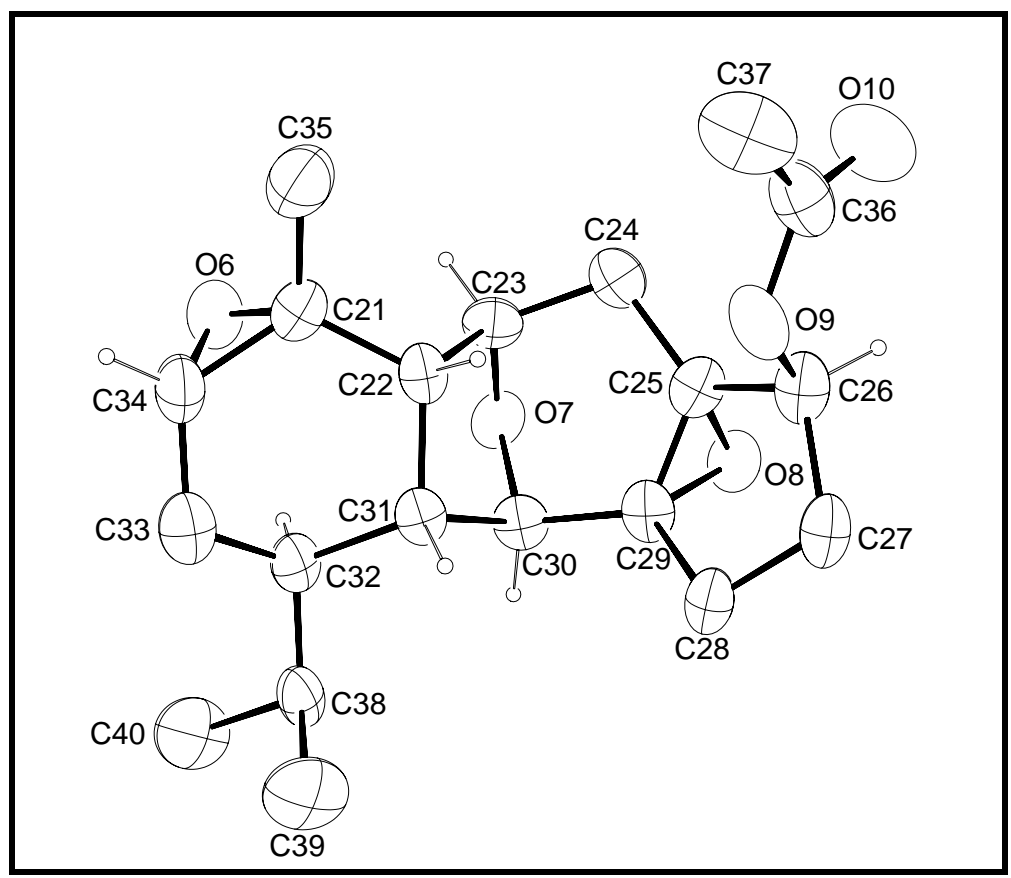

Figure S4. ORTEP drawing of molecule no. 2 of the asymmetric unit with $30 \%$ probability thermal ellipsoids. 


\section{Table S11. Summary of Structure Determination of Compound 18}

Formula:

Formula weight:

Crystal class:

Space group:

Z

Cell constants:

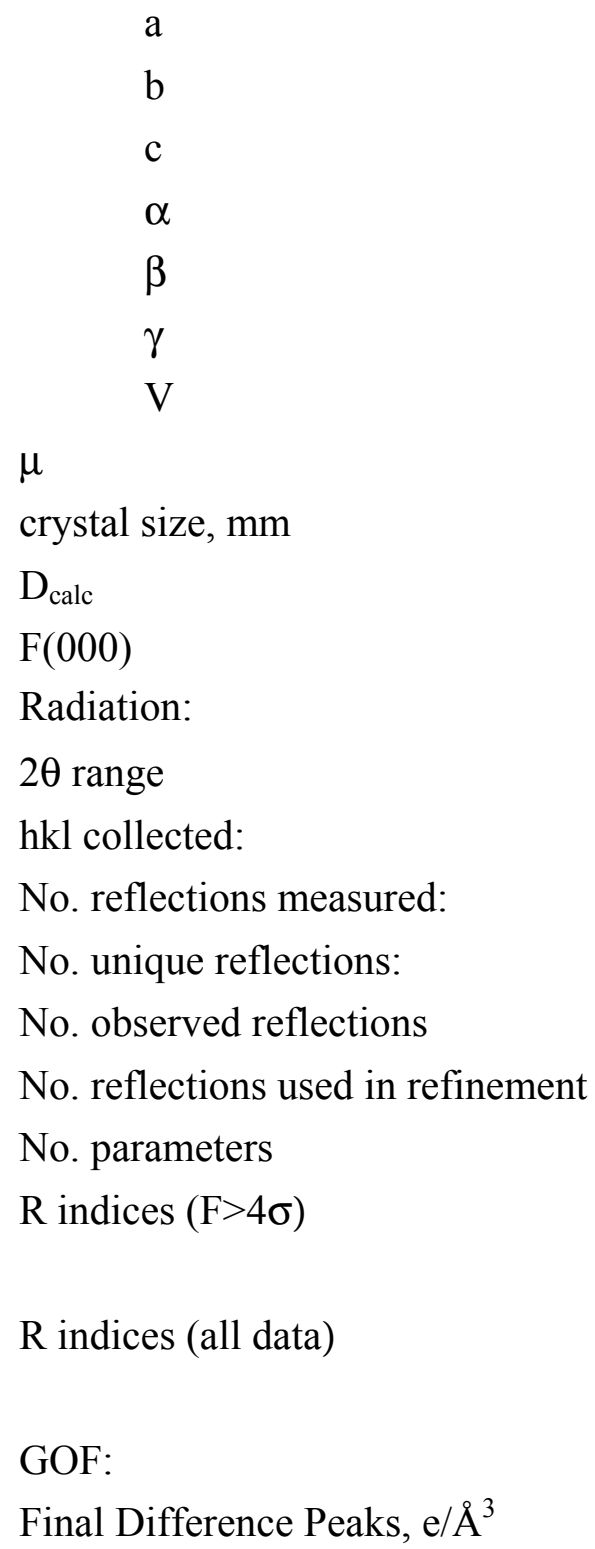

$\mathrm{C}_{20} \mathrm{H}_{28} \mathrm{O}_{6}$

364.42

triclinic

P1 (\#1)

2

13.906(9)A

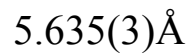

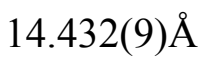

$90.22(3)^{\circ}$

$104.70(2)^{\circ}$

$90.45(3)^{\circ}$

$1093.8(12) \AA^{3}$

$0.81 \mathrm{~cm}^{-1}$

$0.46 \times 0.10 \times 0.01$

$1.107 \mathrm{~g} / \mathrm{cm}^{3}$

392

Mo-K $\mathrm{K}_{\alpha}(\lambda=0.71070 \AA)$

$5.84-44.92^{\circ}$

$-14 \leq \mathrm{h} \leq 14 ;-5 \leq \mathrm{k} \leq 5 ;-15 \leq 1 \leq 11$

4622

$3796\left(\mathrm{R}_{\mathrm{int}}=0.0413\right)$

$2746(\mathrm{~F}>4 \sigma)$

3796

452

$\mathrm{R}_{1}=0.1252$

$\mathrm{wR}_{2}=0.3121$

$\mathrm{R}_{1}=0.1471$

$\mathrm{wR}_{2}=0.3409$

1.322

$+0.943,-0.558$ 
Table S12. Refined Positional Parameters for Compound 18

\begin{tabular}{|c|c|c|c|c|}
\hline Atom & $\mathrm{X}$ & $\mathrm{y}$ & Z & $\mathrm{U}_{\mathrm{eq}}, \AA^{2}$ \\
\hline C1 & $0.6486(9)$ & $0.334(2)$ & $0.4322(9)$ & $0.072(3)$ \\
\hline C2 & $0.6588(7)$ & $0.379(2)$ & $0.3339(7)$ & $0.053(2)$ \\
\hline $\mathrm{H} 2$ & 0.7141 & 0.2824 & 0.3250 & 0.070 \\
\hline C3 & $0.6848(8)$ & $0.646(2)$ & $0.3170(9)$ & $0.064(3)$ \\
\hline $\mathrm{H} 3$ & 0.6897 & 0.7399 & 0.3754 & 0.085 \\
\hline C4 & $0.7780(9)$ & $0.674(2)$ & $0.2838(9)$ & $0.070(3)$ \\
\hline $\mathrm{H} 4 \mathrm{a}$ & 0.8334 & 0.6003 & 0.3290 & 0.093 \\
\hline $\mathrm{H} 4 \mathrm{~b}$ & 0.7936 & 0.8407 & 0.2790 & 0.093 \\
\hline C5 & $0.7603(8)$ & $0.552(2)$ & $0.1846(8)$ & $0.057(3)$ \\
\hline C6 & $0.8372(9)$ & $0.396(2)$ & $0.1501(9)$ & $0.068(3)$ \\
\hline $\mathrm{H} 6$ & 0.8895 & 0.4928 & 0.1341 & 0.090 \\
\hline C7 & $0.7745(9)$ & $0.265(2)$ & $0.0628(9)$ & $0.070(3)$ \\
\hline $\mathrm{H} 7 \mathrm{a}$ & 0.7786 & 0.3463 & 0.0046 & 0.093 \\
\hline $\mathrm{H} 7 \mathrm{~b}$ & 0.7983 & 0.1048 & 0.0604 & 0.093 \\
\hline C8 & $0.6678(8)$ & $0.261(2)$ & $0.0710(8)$ & $0.061(3)$ \\
\hline $\mathrm{H} 8 \mathrm{a}$ & 0.6217 & 0.2759 & 0.0084 & 0.081 \\
\hline $\mathrm{H} 8 \mathrm{~b}$ & 0.6533 & 0.1146 & 0.1000 & 0.081 \\
\hline C9 & $0.6603(8)$ & $0.473(2)$ & $0.1341(8)$ & $0.060(3)$ \\
\hline C10 & $0.5767(8)$ & $0.522(2)$ & $0.1789(7)$ & $0.056(3)$ \\
\hline $\mathrm{H} 10$ & 0.5141 & 0.5456 & 0.1303 & 0.075 \\
\hline C11 & $0.5669(7)$ & $0.321(2)$ & $0.2490(7)$ & $0.055(3)$ \\
\hline $\mathrm{H} 11$ & 0.5752 & 0.1659 & 0.2210 & 0.073 \\
\hline C12 & $0.4664(7)$ & $0.327(2)$ & $0.2779(8)$ & $0.058(3)$ \\
\hline $\mathrm{H} 12$ & 0.4580 & 0.4880 & 0.3000 & 0.077 \\
\hline C13 & $0.4725(10)$ & $0.159(2)$ & $0.3625(10)$ & $0.079(4)$ \\
\hline $\mathrm{H} 13 \mathrm{a}$ & 0.4105 & 0.1658 & 0.3815 & 0.105 \\
\hline $\mathrm{H} 13 \mathrm{~b}$ & 0.4805 & -0.0018 & 0.3421 & 0.105 \\
\hline C14 & $0.5578(9)$ & $0.220(2)$ & $0.4488(10)$ & $0.079(3)$ \\
\hline $\mathrm{H} 14$ & 0.5663 & 0.1182 & 0.5051 & 0.105 \\
\hline C15 & $0.7481(10)$ & $0.303(3)$ & $0.5089(10)$ & $0.096(4)$ \\
\hline $\mathrm{H} 15 \mathrm{a}$ & 0.7349 & 0.2738 & 0.5700 & 0.144 \\
\hline $\mathrm{H} 15 \mathrm{~b}$ & 0.7878 & 0.4442 & 0.5125 & 0.144 \\
\hline $\mathrm{H} 15 \mathrm{c}$ & 0.7833 & 0.1705 & 0.4917 & 0.144 \\
\hline C16 & $0.9698(10)$ & $0.260(2)$ & $0.2841(11)$ & $0.089(4)$ \\
\hline C17 & $1.0022(12)$ & $0.075(3)$ & $0.3494(13)$ & $0.120(6)$ \\
\hline $\mathrm{H} 17 \mathrm{a}$ & 0.9505 & -0.0423 & 0.3421 & 0.180 \\
\hline $\mathrm{H} 17 \mathrm{~b}$ & 1.0178 & 0.1377 & 0.4136 & 0.180 \\
\hline $\mathrm{H} 17 \mathrm{c}$ & 1.0604 & 0.0044 & 0.3373 & 0.180 \\
\hline C18 & $0.3779(8)$ & $0.272(2)$ & $0.1927(9)$ & $0.070(3)$ \\
\hline $\mathrm{H} 18$ & 0.3845 & 0.3770 & 0.1407 & 0.093 \\
\hline C19 & $0.3742(10)$ & $0.019(3)$ & $0.1540(12)$ & $0.101(5)$ \\
\hline $\mathrm{H} 19 \mathrm{a}$ & 0.3175 & -0.0002 & 0.1003 & 0.152 \\
\hline $\mathrm{H} 19 \mathrm{~b}$ & 0.3691 & -0.0910 & 0.2033 & 0.152 \\
\hline $\mathrm{H} 19 \mathrm{c}$ & 0.4337 & -0.0124 & 0.1341 & 0.152 \\
\hline C20 & $0.2788(11)$ & $0.332(3)$ & $0.215(2)$ & $0.115(6)$ \\
\hline $\mathrm{H} 20 \mathrm{a}$ & 0.2251 & 0.2973 & 0.1600 & 0.173 \\
\hline $\mathrm{H} 20 \mathrm{~b}$ & 0.2781 & 0.4978 & 0.2307 & 0.173 \\
\hline $\mathrm{H} 20 \mathrm{c}$ & 0.2710 & 0.2392 & 0.2682 & 0.173 \\
\hline O1 & $0.5779(6)$ & $0.475(2)$ & $0.4662(6)$ & $0.085(2)$ \\
\hline $\mathrm{O} 2$ & $0.6020(5)$ & $0.7259(10)$ & $0.2413(5)$ & $0.059(2)$ \\
\hline
\end{tabular}




\begin{tabular}{|c|c|c|c|c|}
\hline O3 & $0.7049(5)$ & $0.6880(11)$ & $0.1029(5)$ & $0.065(2)$ \\
\hline $\mathrm{O} 4$ & $0.8779(5)$ & $0.2295(12)$ & $0.2269(6)$ & $0.071(2)$ \\
\hline O5 & $1.0187(8)$ & $0.448(2)$ & $0.2736(8)$ & $0.111(4)$ \\
\hline C21 & $0.3758(9)$ & $0.639(2)$ & $0.8666(9)$ & $0.068(3)$ \\
\hline C22 & $0.3635(7)$ & $0.597(2)$ & $0.7616(8)$ & $0.056(3)$ \\
\hline $\mathrm{H} 22$ & 0.3085 & 0.6963 & 0.7269 & 0.075 \\
\hline C23 & $0.3373(8)$ & $0.330(2)$ & $0.7286(9)$ & $0.063(3)$ \\
\hline $\mathrm{H} 23$ & 0.3321 & 0.2346 & 0.7838 & 0.084 \\
\hline C24 & $0.2428(8)$ & $0.310(2)$ & $0.6513(9)$ & $0.065(3)$ \\
\hline $\mathrm{H} 24 \mathrm{a}$ & 0.1891 & 0.3857 & 0.6715 & 0.086 \\
\hline$H 24 b$ & 0.2252 & 0.1438 & 0.6378 & 0.086 \\
\hline C25 & $0.2590(9)$ & $0.431(2)$ & $0.5616(8)$ & $0.063(3)$ \\
\hline C26 & $0.1835(9)$ & $0.584(2)$ & $0.4937(9)$ & $0.071(3)$ \\
\hline H26 & 0.1325 & 0.4874 & 0.4503 & 0.094 \\
\hline C27 & $0.2457(9)$ & $0.725(2)$ & $0.4385(9)$ & $0.074(3)$ \\
\hline $\mathrm{H} 27 \mathrm{a}$ & 0.2374 & 0.6577 & 0.3750 & 0.098 \\
\hline $\mathrm{H} 27 \mathrm{~b}$ & 0.2240 & 0.8891 & 0.4317 & 0.098 \\
\hline C28 & $0.3535(8)$ & $0.715(2)$ & $0.4934(8)$ & $0.062(3)$ \\
\hline $\mathrm{H} 28 \mathrm{a}$ & 0.3960 & 0.6930 & 0.4503 & 0.082 \\
\hline $\mathrm{H} 28 \mathrm{~b}$ & 0.3735 & 0.8595 & 0.5302 & 0.082 \\
\hline C29 & $0.3593(8)$ & $0.507(2)$ & $0.5582(8)$ & $0.057(3)$ \\
\hline C30 & $0.4445(8)$ & $0.457(2)$ & $0.6460(8)$ & $0.057(3)$ \\
\hline H3O & 0.5069 & 0.4327 & 0.6277 & 0.076 \\
\hline C31 & $0.4557(7)$ & $0.652(2)$ & $0.7208(7)$ & $0.052(2)$ \\
\hline H31 & 0.4470 & 0.8062 & 0.6890 & 0.069 \\
\hline C32 & $0.5571(8)$ & $0.649(2)$ & $0.7976(8)$ & $0.059(3)$ \\
\hline H32 & 0.5668 & 0.4877 & 0.8229 & 0.078 \\
\hline C33 & $0.5529(10)$ & $0.812(2)$ & $0.8806(9)$ & $0.077(4)$ \\
\hline H33a & 0.6161 & 0.8077 & 0.9284 & 0.102 \\
\hline $\mathrm{H} 33 \mathrm{~b}$ & 0.5425 & 0.9740 & 0.8576 & 0.102 \\
\hline C34 & $0.4719(9)$ & $0.743(2)$ & $0.9258(9)$ & $0.078(4)$ \\
\hline H34 & 0.4665 & 0.8401 & 0.9807 & 0.104 \\
\hline C35 & $0.2823(11)$ & $0.670(3)$ & $0.8987(11)$ & $0.102(5)$ \\
\hline H35a & 0.2988 & 0.6966 & 0.9667 & 0.152 \\
\hline$H 35 b$ & 0.2415 & 0.5303 & 0.8831 & 0.152 \\
\hline $\mathrm{H} 35 \mathrm{c}$ & 0.2467 & 0.8045 & 0.8668 & 0.152 \\
\hline C36 & $0.0482(11)$ & $0.702(2)$ & $0.5570(11)$ & $0.083(4)$ \\
\hline C37 & $0.0132(12)$ & $0.882(3)$ & $0.615(2)$ & $0.112(5)$ \\
\hline $\mathrm{H} 37 \mathrm{a}$ & 0.0637 & 1.0014 & 0.6356 & 0.168 \\
\hline H37b & -0.0009 & 0.8074 & 0.6698 & 0.168 \\
\hline H37c & -0.0462 & 0.9536 & 0.5770 & 0.168 \\
\hline C38 & $0.6426(8)$ & $0.705(2)$ & $0.7533(8)$ & $0.067(3)$ \\
\hline H38 & 0.6355 & 0.6013 & 0.6972 & 0.090 \\
\hline C39 & $0.6481(13)$ & $0.968(3)$ & $0.7192(14)$ & $0.112(5)$ \\
\hline $\mathrm{H} 39 \mathrm{a}$ & 0.7038 & 0.9859 & 0.6918 & 0.168 \\
\hline H39b & 0.6560 & 1.0735 & 0.7730 & 0.168 \\
\hline $\mathrm{H} 39 \mathrm{c}$ & 0.5879 & 1.0050 & 0.6721 & 0.168 \\
\hline C40 & $0.7459(11)$ & $0.651(3)$ & $0.8241(13)$ & $0.116(6)$ \\
\hline $\mathrm{H} 40 \mathrm{a}$ & 0.7981 & 0.6871 & 0.7937 & 0.174 \\
\hline $\mathrm{H} 40 \mathrm{~b}$ & 0.7486 & 0.4867 & 0.8414 & 0.174 \\
\hline $\mathrm{H} 40 \mathrm{c}$ & 0.7541 & 0.7475 & 0.8807 & 0.174 \\
\hline O6 & $0.4498(6)$ & $0.494(2)$ & $0.9317(6)$ & $0.083(2)$ \\
\hline $\mathrm{O} 7$ & $0.4187(5)$ & $0.2502(11)$ & $0.6944(5)$ & $0.062(2)$ \\
\hline O8 & $0.3131(6)$ & $0.2935(12)$ & $0.5057(5)$ & $0.067(2)$ \\
\hline O9 & $0.1399(6)$ & $0.7459(13)$ & $0.5490(6)$ & $0.075(2)$ \\
\hline
\end{tabular}




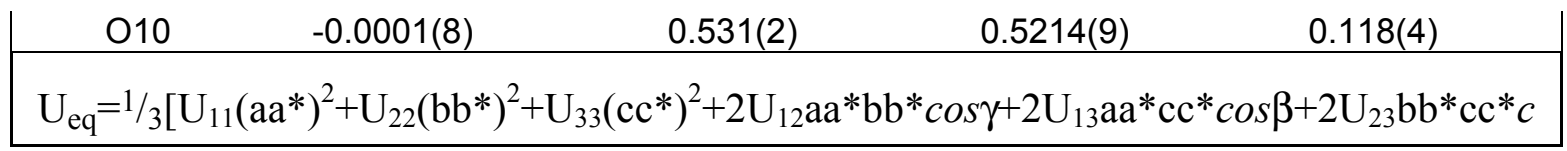

Table S13. Refined Thermal Parameters (U's) for Compound 18

\begin{tabular}{|c|c|c|c|c|c|c|}
\hline Atom & $\mathrm{U}_{11}$ & $\mathrm{U}_{22}$ & $\mathrm{U}_{33}$ & $\mathrm{U}_{23}$ & $\mathrm{U}_{13}$ & $\mathrm{U}_{12}$ \\
\hline C1 & $0.078(8)$ & $0.069(8)$ & $0.063(8)$ & $0.006(6)$ & $0.004(6)$ & $0.005(6)$ \\
\hline C2 & $0.062(6)$ & $0.043(6)$ & $0.049(6)$ & $0.007(4)$ & $0.007(5)$ & $-0.003(4)$ \\
\hline C3 & $0.074(7)$ & $0.035(6)$ & $0.080(8)$ & $0.009(5)$ & $0.014(6)$ & $0.007(5)$ \\
\hline $\mathrm{C} 4$ & $0.078(8)$ & $0.037(6)$ & $0.081(8)$ & $0.014(5)$ & $-0.008(6)$ & $-0.003(5)$ \\
\hline C5 & $0.067(7)$ & $0.030(5)$ & $0.068(7)$ & $0.008(5)$ & $0.009(5)$ & $0.003(4)$ \\
\hline C6 & $0.077(7)$ & $0.055(7)$ & $0.068(8)$ & $0.017(5)$ & $0.013(6)$ & $-0.006(5)$ \\
\hline $\mathrm{C} 7$ & $0.088(9)$ & $0.059(7)$ & $0.059(7)$ & $0.004(5)$ & $0.014(7)$ & $-0.001(5)$ \\
\hline $\mathrm{C} 8$ & $0.077(7)$ & $0.047(6)$ & $0.053(7)$ & $0.003(5)$ & $0.004(5)$ & $0.009(5)$ \\
\hline C9 & $0.085(8)$ & $0.021(5)$ & $0.065(7)$ & $0.014(4)$ & $0.001(6)$ & $0.006(4)$ \\
\hline C10 & $0.083(7)$ & $0.029(5)$ & $0.052(6)$ & $0.007(4)$ & $0.007(6)$ & $0.019(4)$ \\
\hline C11 & $0.064(6)$ & $0.039(5)$ & $0.060(7)$ & $0.006(4)$ & $0.010(5)$ & $0.007(4)$ \\
\hline C12 & $0.053(6)$ & $0.051(6)$ & $0.070(8)$ & $0.000(5)$ & $0.017(6)$ & $0.003(4)$ \\
\hline C13 & $0.100(9)$ & $0.045(7)$ & $0.094(10)$ & $0.012(6)$ & $0.025(8)$ & $0.012(6)$ \\
\hline C14 & $0.078(8)$ & $0.069(8)$ & $0.089(9)$ & $0.012(6)$ & $0.020(7)$ & $-0.008(6)$ \\
\hline C15 & $0.075(8)$ & $0.134(13)$ & $0.066(9)$ & $0.029(8)$ & $-0.006(7)$ & $-0.014(7)$ \\
\hline C16 & $0.077(9)$ & $0.068(9)$ & $0.100(11)$ & $0.001(7)$ & $-0.018(8)$ & $0.011(7)$ \\
\hline C17 & $0.126(12)$ & $0.063(9)$ & $0.123(12)$ & $0.013(8)$ & $-0.059(10)$ & $-0.002(8)$ \\
\hline C18 & $0.081(8)$ & $0.050(7)$ & $0.071(8)$ & $0.001(5)$ & $0.005(6)$ & $-0.007(5)$ \\
\hline C19 & $0.077(8)$ & $0.090(10)$ & $0.118(12)$ & $-0.043(9)$ & $-0.012(8)$ & $0.002(7)$ \\
\hline C20 & $0.096(11)$ & $0.108(12)$ & $0.15(2)$ & $-0.021(11)$ & $0.039(11)$ & $0.010(8)$ \\
\hline O1 & $0.093(6)$ & $0.089(7)$ & $0.076(6)$ & $-0.012(4)$ & $0.031(5)$ & $0.009(4)$ \\
\hline $\mathrm{O} 2$ & $0.074(4)$ & $0.030(4)$ & $0.068(5)$ & $-0.001(3)$ & $0.007(4)$ & $0.002(3)$ \\
\hline $\mathrm{O} 3$ & $0.079(5)$ & $0.041(4)$ & $0.068(5)$ & $0.015(3)$ & $0.005(4)$ & $0.003(3)$ \\
\hline $\mathrm{O} 4$ & $0.074(5)$ & $0.045(4)$ & $0.081(5)$ & $0.009(3)$ & $-0.005(4)$ & $0.008(3)$ \\
\hline O5 & $0.094(7)$ & $0.084(7)$ & $0.132(9)$ & $0.010(6)$ & $-0.014(6)$ & $-0.025(5)$ \\
\hline $\mathrm{C} 21$ & $0.084(8)$ & $0.066(7)$ & $0.056(7)$ & $0.000(5)$ & $0.020(6)$ & $0.004(6)$ \\
\hline $\mathrm{C} 22$ & $0.062(6)$ & $0.044(6)$ & $0.055(7)$ & $0.000(5)$ & $0.001(5)$ & $0.004(4)$ \\
\hline $\mathrm{C} 23$ & $0.070(7)$ & $0.046(6)$ & $0.078(8)$ & $0.009(5)$ & $0.024(6)$ & $-0.002(5)$ \\
\hline $\mathrm{C} 24$ & $0.071(7)$ & $0.041(6)$ & $0.078(8)$ & $-0.010(5)$ & $0.013(6)$ & $-0.005(5)$ \\
\hline C25 & $0.081(8)$ & $0.046(6)$ & $0.060(7)$ & $0.003(5)$ & $0.016(6)$ & $0.010(5)$ \\
\hline $\mathrm{C} 26$ & $0.093(8)$ & $0.046(6)$ & $0.064(7)$ & $-0.002(5)$ & $0.002(6)$ & $0.003(5)$ \\
\hline $\mathrm{C} 27$ & $0.091(8)$ & $0.068(8)$ & $0.052(7)$ & $-0.006(5)$ & $-0.001(6)$ & $0.007(6)$ \\
\hline $\mathrm{C} 28$ & $0.090(8)$ & $0.038(6)$ & $0.049(6)$ & $-0.001(4)$ & $0.002(6)$ & $0.002(5)$ \\
\hline C29 & $0.075(7)$ & $0.023(5)$ & $0.066(7)$ & $-0.005(4)$ & $0.007(6)$ & $0.005(4)$ \\
\hline C30 & $0.070(7)$ & $0.031(5)$ & $0.069(7)$ & $0.004(4)$ & $0.014(6)$ & $0.005(4)$ \\
\hline C31 & $0.062(6)$ & $0.026(5)$ & $0.060(6)$ & $0.008(4)$ & $0.003(5)$ & $0.006(4)$ \\
\hline C32 & $0.065(6)$ & $0.045(6)$ & $0.057(6)$ & $0.013(5)$ & $-0.001(5)$ & $0.006(4)$ \\
\hline C33 & $0.087(8)$ & $0.060(7)$ & $0.073(9)$ & $0.001(6)$ & $0.000(7)$ & $0.008(6)$ \\
\hline C34 & $0.083(9)$ & $0.084(9)$ & $0.058(7)$ & $-0.015(6)$ & $0.003(7)$ & $-0.008(6)$ \\
\hline C35 & $0.109(11)$ & $0.122(13)$ & $0.075(10)$ & $0.005(9)$ & $0.025(9)$ & $0.005(9)$ \\
\hline C36 & $0.077(9)$ & $0.056(8)$ & $0.100(10)$ & $0.007(7)$ & $-0.005(7)$ & $-0.007(6)$ \\
\hline C37 & $0.103(11)$ & $0.073(10)$ & $0.17(2)$ & $0.003(10)$ & $0.042(11)$ & $0.006(7)$ \\
\hline C38 & $0.075(7)$ & $0.052(7)$ & $0.060(7)$ & $0.011(5)$ & $-0.010(6)^{\prime}$ & $-0.009(5)$ \\
\hline C39 & $0.124(12)$ & $0.076(10)$ & $0.135(14)$ & $0.045(9)$ & $0.030(11)$ & $0.015(8)$ \\
\hline $\mathrm{C} 40$ & $0.095(10)$ & $0.121(13)$ & $0.119(13)$ & $0.057(11)$ & $0.001(9)$ & $0.020(9)$ \\
\hline
\end{tabular}




\begin{tabular}{|c|c|c|c|c|c|c|}
\hline O6 & $0.094(6)$ & $0.077(6)$ & $0.067(5)$ & $0.015(4)$ & $-0.001(4)$ & $-0.003(4)$ \\
\hline $\mathrm{O} 7$ & $0.088(5)$ & $0.036(4)$ & $0.060(5)$ & $0.006(3)$ & $0.018(4)$ & $0.006(3)$ \\
\hline O8 & $0.089(5)$ & $0.046(4)$ & $0.062(5)$ & $-0.009(3)$ & $0.013(4)$ & $0.001(3)$ \\
\hline O9 & $0.066(5)$ & $0.057(5)$ & $0.088(6)$ & $-0.008(4)$ & $-0.007(4)$ & $-0.003(4)$ \\
\hline 010 & $0.096(7)$ & $0.111(9)$ & $0.147(11)$ & $-0.029(8)$ & $0.031(7)$ & $-0.019(6)$ \\
\hline
\end{tabular}

The form of the anisotropic displacement parameter is:

$\exp \left[-2 \pi^{2}\left(a^{* 2} U_{11} h^{2}+b^{* 2} U_{22} k^{2}+c^{* 2} U_{33} l^{2}+2 b * c * U_{23} k l+2 a * c * U_{13} h l+2 a * b * U_{12} h k\right)\right]$.

Table S14. Bond Distances in Compound 18, $\AA$

\begin{tabular}{|llllll|}
\hline C1-O1 & $1.448(14)$ & C1-C2 & $1.48(2)$ & C1-C14 & $1.49(2)$ \\
C1-C15 & $1.55(2)$ & C2-C11 & $1.56(2)$ & C2-C3 & $1.578(14)$ \\
C3-O2 & $1.447(13)$ & C3-C4 & $1.50(2)$ & C4-C5 & $1.55(2)$ \\
C5-O3 & $1.457(12)$ & C5-C9 & $1.46(2)$ & C5-C6 & $1.56(2)$ \\
C6-O4 & $1.458(13)$ & C6-C7 & $1.52(2)$ & C7-C8 & $1.52(2)$ \\
C8-C9 & $1.52(2)$ & C9-O3 & $1.481(12)$ & C9-C10 & $1.50(2)$ \\
C10-O2 & $1.442(12)$ & C10-C11 & $1.551(13)$ & C11-C12 & $1.56(2)$ \\
C12-C13 & $1.53(2)$ & C12-C18 & $1.53(2)$ & C13-C14 & $1.52(2)$ \\
C14-O1 & $1.47(2)$ & C16-O5 & $1.28(2)$ & C16-O4 & $1.34(2)$ \\
C16-C17 & $1.41(2)$ & C18-C19 & $1.53(2)$ & C18-C20 & $1.53(2)$ \\
C21-O6 & $1.462(14)$ & C21-C35 & $1.50(2)$ & C21-C22 & $1.50(2)$ \\
C21-C34 & $1.50(2)$ & C22-C31 & $1.569(14)$ & C22-C23 & $1.59(2)$ \\
C23-O7 & $1.421(13)$ & C23-C24 & $1.50(2)$ & C24-C25 & $1.53(2)$ \\
C25-O8 & $1.458(13)$ & C25-C29 & $1.47(2)$ & C25-C26 & $1.52(2)$ \\
C26-O9 & $1.443(14)$ & C26-C27 & $1.54(2)$ & C27-C28 & $1.51(2)$ \\
C28-C29 & $1.49(2)$ & C29-O8 & $1.470(12)$ & C29-C30 & $1.53(2)$ \\
C30-O7 & $1.449(12)$ & C30-C31 & $1.52(2)$ & C31-C32 & $1.557(14)$ \\
C32-C38 & $1.52(2)$ & C32-C33 & $1.52(2)$ & C33-C34 & $1.49(2)$ \\
C34-O6 & $1.44(2)$ & C36-O10 & $1.21(2)$ & C36-O9 & $1.33(2)$ \\
C36-C37 & $1.47(2)$ & C38-C40 & $1.57(2)$ & C38-C39 & $1.57(2)$ \\
\hline
\end{tabular}

Table S15. Bond Angles in Compound 18, ${ }^{\circ}$

\begin{tabular}{|llllll|}
\hline O1-C1-C2 & $117.7(10)$ & O1-C1-C14 & $60.2(8)$ & C2-C1-C14 & $121.4(11)$ \\
O1-C1-C15 & $112.7(11)$ & C2-C1-C15 & $114.8(10)$ & C14-C1-C15 & $118.3(10)$ \\
C1-C2-C11 & $117.2(9)$ & C1-C2-C3 & $113.3(9)$ & C11-C2-C3 & $104.1(8)$ \\
O2-C3-C4 & $108.3(9)$ & O2-C3-C2 & $104.6(8)$ & C4-C3-C2 & $113.2(8)$ \\
C3-C4-C5 & $107.7(9)$ & O3-C5-C9 & $61.0(6)$ & O3-C5-C4 & $116.2(8)$ \\
C9-C5-C4 & $120.0(9)$ & O3-C5-C6 & $108.0(8)$ & C9-C5-C6 & $108.3(9)$ \\
C4-C5-C6 & $125.5(9)$ & O4-C6-C7 & $110.6(9)$ & O4-C6-C5 & $106.9(8)$ \\
C7-C6-C5 & $103.7(9)$ & C8-C7-C6 & $107.8(10)$ & C9-C8-C7 & $104.9(9)$ \\
C5-C9-O3 & $59.4(6)$ & C5-C9-C10 & $118.3(10)$ & O3-C9-C10 & $114.1(8)$ \\
C5-C9-C8 & $109.3(9)$ & O3-C9-C8 & $111.1(9)$ & C10-C9-C8 & $126.1(9)$ \\
O2-C10-C9 & $109.1(9)$ & O2-C10-C11 & $102.8(8)$ & C9-C10-C11 & $110.5(7)$ \\
C10-C11-C12 & $112.8(8)$ & C10-C11-C2 & $100.0(8)$ & C12-C11-C2 & $113.4(9)$ \\
C13-C12-C18 & $113.1(9)$ & C13-C12-C11 & $108.9(8)$ & C18-C12-C11 & $111.9(9)$ \\
C14-C13-C12 & $113.2(10)$ & O1-C14-C1 & $58.6(7)$ & O1-C14-C13 & $115.1(10)$ \\
C1-C14-C13 & $118.5(11)$ & O5-C16-O4 & $118.4(12)$ & O5-C16-C17 & $127.0(13)$ \\
O4-C16-C17 & $114.5(12)$ & C19-C18-C12 & $114.7(9)$ & C19-C18-C20 & $109.9(11)$ \\
C12-C18-C20 & $111.7(11)$ & C1-O1-C14 & $61.1(8)$ & C10-O2-C3 & $103.9(7)$ \\
C5-O3-C9 & $59.7(6)$ & C16-O4-C6 & $120.9(9)$ & O6-C21-C35 & $113.4(10)$ \\
O6-C21-C22 & $116.4(9)$ & C35-C21-C22 & $116.5(11)$ & O6-C21-C34 & $58.1(8)$ \\
C35-C21-C34 & $119.5(11)$ & C22-C21-C34 & $119.3(10)$ & C21-C22-C31 & $117.1(9)$ \\
C21-C22-C23 & $113.9(9)$ & C31-C22-C23 & $102.4(8)$ & O7-C23-C24 & $110.2(10)$ \\
\hline
\end{tabular}




\begin{tabular}{|llllll|}
\hline O7-C23-C22 & $105.4(8)$ & C24-C23-C22 & $112.2(8)$ & C23-C24-C25 & $108.1(9)$ \\
O8-C25-C29 & $60.3(6)$ & O8-C25-C26 & $108.7(9)$ & C29-C25-C26 & $108.7(9)$ \\
O8-C25-C24 & $115.9(8)$ & C29-C25-C24 & $119.9(10)$ & C26-C25-C24 & $125.3(10)$ \\
O9-C26-C25 & $109.1(9)$ & O9-C26-C27 & $109.4(9)$ & C25-C26-C27 & $104.2(10)$ \\
C28-C27-C26 & $108.5(10)$ & C29-C28-C27 & $104.9(9)$ & C25-C29-O8 & $59.5(7)$ \\
C25-C29-C28 & $110.1(8)$ & O8-C29-C28 & $111.7(9)$ & C25-C29-C30 & $117.5(9)$ \\
O8-C29-C30 & $114.6(7)$ & C28-C29-C30 & $125.3(9)$ & O7-C30-C31 & $103.2(8)$ \\
O7-C30-C29 & $108.7(9)$ & C31-C30-C29 & $111.7(7)$ & C30-C31-C32 & $113.9(8)$ \\
C30-C31-C22 & $100.6(8)$ & C32-C31-C22 & $114.1(8)$ & C38-C32-C33 & $113.7(9)$ \\
C38-C32-C31 & $111.2(8)$ & C33-C32-C31 & $109.9(8)$ & C34-C33-C32 & $112.0(10)$ \\
O6-C34-C33 & $118.2(10)$ & O6-C34-C21 & $59.5(8)$ & C33-C34-C21 & $120.8(11)$ \\
O10-C36-O9 & $123.1(14)$ & O10-C36-C37 & $124.0(14)$ & O9-C36-C37 & $113.0(12)$ \\
C32-C38-C40 & $111.7(9)$ & C32-C38-C39 & $115.3(10)$ & C40-C38-C39 & $106.6(11)$ \\
C34-O6-C21 & $62.5(8)$ & C23-O7-C30 & $103.0(7)$ & C25-O8-C29 & $60.2(7)$ \\
C36-O9-C26 & $118.9(9)$ & & & & \\
\hline
\end{tabular}




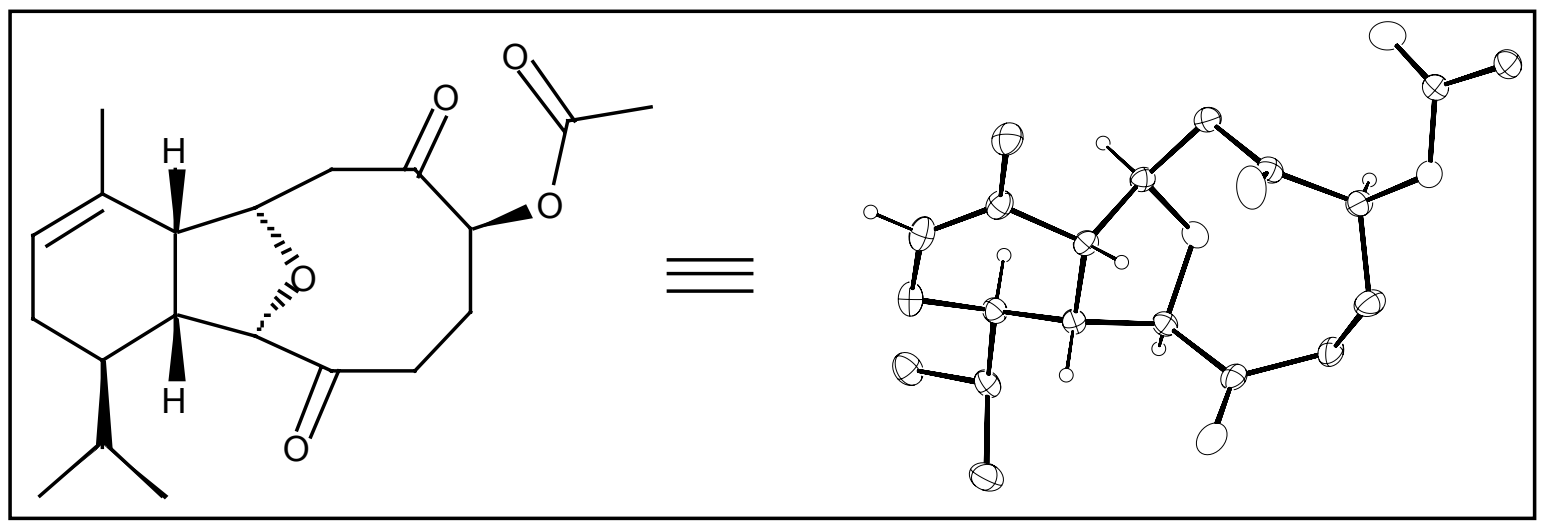

\section{X-ray Structure Determination of Compound 16}

Compound 16, $\mathrm{C}_{20} \mathrm{H}_{28} \mathrm{O}_{5}$, crystallizes in the orthorhombic space group $\mathrm{P} 2{ }_{1} 2_{1} 2_{1}$ (systematic absences $\mathrm{h} 00: \mathrm{h}=\mathrm{odd}, 0 \mathrm{k} 0: \mathrm{k}=\mathrm{odd}$, and 001: $\mathrm{l}=\mathrm{odd}$ ) with $\mathrm{a}=10.3569(13) \AA$, $\mathrm{b}=11.784(2) \AA, \mathrm{c}=14.868(2) \AA, \mathrm{V}=1814.7(4) \AA^{3}, \mathrm{Z}=4$ and $\mathrm{d}_{\mathrm{calc}}=1.275 \mathrm{~g} / \mathrm{cm}^{3}$. X-ray intensity data were collected on a Rigaku Mercury CCD area detector employing graphite-monochromated Mo- $\mathrm{K}_{\alpha}$ radiation $(\lambda=0.71069 \AA)$ at a temperature of $143^{\circ} \mathrm{K}$. Indexing was performed from a series of four $0.5^{\circ}$ oscillation images with exposures of 30 seconds per frame. A hemisphere of data was collected using 30 second exposures and a crystal-to-detector distance of $40 \mathrm{~mm}$. A total of 440 images were collected: one sweep was performed using $\phi$-scans from $-90^{\circ}$ to $+90^{\circ}$ in $0.5^{\circ}$ steps at $\omega=0^{\circ}$ and $\chi=0^{\circ}$ with a detector swing angle of $-15^{\circ}$; a second sweep was done using $\omega$-scans from $-20^{\circ}$ to $+20^{\circ}$ in $0.5^{\circ}$ steps at $\chi=-90^{\circ}$ and $\phi=0^{\circ}$ with a detector swing angle of $-15^{\circ}$. Oscillation images were processed using CrystalClear ${ }^{25}$, producing a listing of unaveraged $\mathrm{F}^{2}$ and $\sigma\left(\mathrm{F}^{2}\right)$ values which were then passed to the CrystalStructure ${ }^{26}$ program package for further processing and structure solution on a Dell Pentium III computer. A total of 8994 reflections were measured over the ranges $5.24 \leq 2 \theta \leq 50.7^{\circ},-12 \leq \mathrm{h} \leq 10,-10 \leq \mathrm{k} \leq 14$, $-16 \leq 1 \leq 17$ yielding 3291 unique reflections $\left(\mathrm{R}_{\text {int }}=0.0168\right)$. The intensity data were corrected for Lorentz and polarization effects and for absorption. using REQAB ${ }^{27}$ (minimum and maximum transmission $0.846,1.000$ ). 
The structure was solved by direct methods $\left(\operatorname{SIR} 97^{28}\right)$. Refinement was by full-matrix least squares based on $\mathrm{F}^{2}$ using SHELXL-9 $7^{29}$. All reflections were used during refinement $\left(\mathrm{F}^{2}\right.$,s that were experimentally negative were replaced by $\left.\mathrm{F}^{2}=0\right)$. The weighting scheme used was $\mathrm{w}=1 /\left[\sigma^{2}\left(\mathrm{~F}_{\mathrm{o}}^{2}\right)+0.0493 \mathrm{P}^{2}+0.2879 \mathrm{P}\right]$ where $\mathrm{P}=\left(\mathrm{F}_{\mathrm{o}}^{2}+2 \mathrm{~F}_{\mathrm{c}}^{2}\right) / 3$. Non-hydrogen atoms were refined anisotropically and hydrogen atoms were refined using a "riding" model. Refinement converged to $R_{1}=0.0343$ and $w_{2}=0.0835$ for 3098 reflections for which $F>4 \sigma(F)$ and $\mathrm{R}_{1}=0.0377, \mathrm{wR}_{2}=0.0858$ and $\mathrm{GOF}=1.030$ for all 3291 unique, non-zero reflections and 230 variables $^{30}$. The maximum $\Delta / \sigma$ in the final cycle of least squares was 0.020 and the two most prominent peaks in the final difference Fourier were +0.148 and $-0.164 \mathrm{e} / \AA^{3}$.

Table S16. lists cell information, data collection parameters, and refinement data. Final positional and equivalent isotropic thermal parameters are given in Table S17. Anisotropic thermal parameters are in Table S18. Tables S19 and S20 list bond distances and bond angles. Figure S5. is an ORTEP ${ }^{31}$ representation of the molecule with $30 \%$ probability thermal ellipsoids displayed.

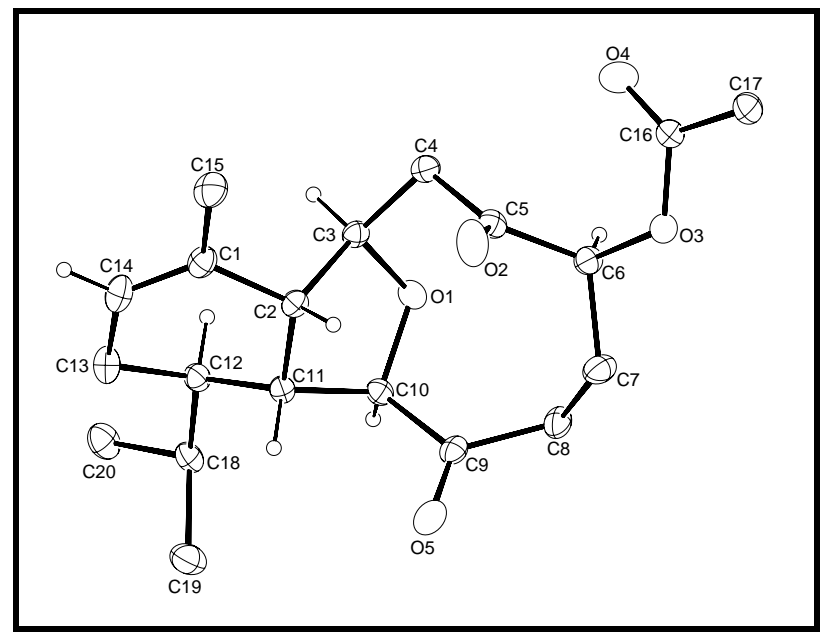

Figure S5. ORTEP drawing of the title compound with 30\% probability thermal ellipsoids. 
Table S16. Summary of Structure Determination of Compound 16

Formula:

Formula weight:

Crystal class:

Space group:

Z

Cell constants:

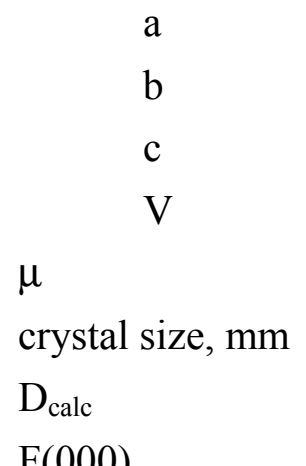

Radiation:

$2 \theta$ range

hkl collected:

No. reflections measured:

No. unique reflections:

No. observed reflections

No. reflections used in refinement

No. parameters

$R$ indices $(F>4 \sigma)$

$\mathrm{R}$ indices (all data)

GOF:

Final Difference Peaks, e/ $\AA^{3}$
$\mathrm{C}_{20} \mathrm{H}_{28} \mathrm{O}_{5}$

348.42

orthorhombic

$\mathrm{P} 22_{1} 2_{1}$ (\#19)

4

$10.3569(13) \AA$

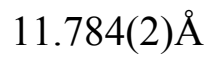

$14.868(2) \AA$

$1814.7(4) \AA^{3}$

$0.90 \mathrm{~cm}^{-1}$

$0.30 \times 0.24 \times 0.12$

$1.275 \mathrm{~g} / \mathrm{cm}^{3}$

752

$\operatorname{Mo}^{-K_{\alpha}}(\lambda=0.71070 \AA)$

$5.24-50.7^{\circ}$

$-12 \leq \mathrm{h} \leq 10 ;-10 \leq \mathrm{k} \leq 14 ;-16 \leq 1 \leq 17$

8994

$3291\left(\mathrm{R}_{\mathrm{int}}=0.0168\right)$

$3098(\mathrm{~F}>4 \sigma)$

3291

230

$\mathrm{R}_{1}=0.0343$

$\mathrm{wR}_{2}=0.0835$

$\mathrm{R}_{1}=0.0377$

$\mathrm{wR}_{2}=0.0858$

1.030

$+0.148,-0.164$ 
Table S17. Refined Positional Parameters for Compound 16

\begin{tabular}{|c|c|c|c|c|}
\hline Atom & $\mathrm{X}$ & $\mathrm{y}$ & $\mathrm{Z}$ & $\mathrm{U}_{\mathrm{eq}}, \AA^{2}$ \\
\hline C1 & $0.3146(2)$ & $0.03218(14)$ & $0.54845(12)$ & $0.0303(4)$ \\
\hline $\mathrm{C} 2$ & $0.35771(14)$ & $0.15195(13)$ & $0.52567(11)$ & $0.0244(3)$ \\
\hline $\mathrm{H} 2$ & 0.2906 & 0.1870 & 0.4885 & 0.032 \\
\hline C3 & $0.48654(14)$ & $0.16056(13)$ & $0.47461(11)$ & $0.0248(3)$ \\
\hline $\mathrm{H} 3$ & 0.5459 & 0.1029 & 0.4979 & 0.033 \\
\hline C4 & $0.4823(2)$ & $0.15195(14)$ & $0.37212(11)$ & $0.0298(4)$ \\
\hline $\mathrm{H} 4 \mathrm{a}$ & 0.5684 & 0.1644 & 0.3484 & 0.040 \\
\hline $\mathrm{H} 4 \mathrm{~b}$ & 0.4558 & 0.0759 & 0.3553 & 0.040 \\
\hline C5 & $0.3913(2)$ & $0.23623(14)$ & $0.33043(10)$ & $0.0273(3)$ \\
\hline C6 & $0.4398(2)$ & $0.35464(14)$ & $0.30380(10)$ & $0.0279(3)$ \\
\hline $\mathrm{H} 6$ & 0.5338 & 0.3588 & 0.3103 & 0.037 \\
\hline $\mathrm{C} 7$ & $0.3761(2)$ & $0.4527(2)$ & $0.35371(12)$ & $0.0377(4)$ \\
\hline $\mathrm{H} 7 \mathrm{a}$ & 0.2856 & 0.4345 & 0.3623 & 0.050 \\
\hline$H 7 b$ & 0.3802 & 0.5196 & 0.3158 & 0.050 \\
\hline C8 & $0.4346(2)$ & $0.48278(14)$ & $0.44593(11)$ & $0.0338(4)$ \\
\hline H8a & 0.5269 & 0.4693 & 0.4437 & 0.045 \\
\hline $\mathrm{H} 8 \mathrm{~b}$ & 0.4217 & 0.5631 & 0.4569 & 0.045 \\
\hline C9 & $0.3787(2)$ & $0.41695(12)$ & $0.52411(11)$ & $0.0270(3)$ \\
\hline C10 & $0.46059(14)$ & $0.32275(13)$ & $0.56557(10)$ & $0.0243(3)$ \\
\hline $\mathrm{H} 10$ & 0.5176 & 0.3552 & 0.6115 & 0.032 \\
\hline C11 & $0.37994(14)$ & $0.22791(13)$ & $0.60733(10)$ & $0.0234(3)$ \\
\hline $\mathrm{H} 11$ & 0.2971 & 0.2589 & 0.6276 & 0.031 \\
\hline C12 & $0.44488(14)$ & $0.16479(13)$ & $0.68544(10)$ & $0.0262(3)$ \\
\hline $\mathrm{H} 12$ & 0.5251 & 0.1323 & 0.6618 & 0.035 \\
\hline C13 & $0.3583(2)$ & $0.0648(2)$ & $0.71250(12)$ & $0.0371(4)$ \\
\hline $\mathrm{H} 13 \mathrm{a}$ & 0.4060 & 0.0152 & 0.7527 & 0.049 \\
\hline $\mathrm{H} 13 \mathrm{~b}$ & 0.2841 & 0.0935 & 0.7451 & 0.049 \\
\hline C14 & $0.3122(2)$ & $-0.0028(2)$ & $0.63340(13)$ & $0.0373(4)$ \\
\hline $\mathrm{H} 14$ & 0.2794 & -0.0749 & 0.6446 & 0.050 \\
\hline C15 & $0.2685(2)$ & $-0.0392(2)$ & $0.47149(13)$ & $0.0403(4)$ \\
\hline $\mathrm{H} 15 \mathrm{a}$ & 0.3408 & -0.0610 & 0.4350 & 0.061 \\
\hline $\mathrm{H} 15 \mathrm{~b}$ & 0.2085 & 0.0035 & 0.4358 & 0.061 \\
\hline $\mathrm{H} 15 \mathrm{c}$ & 0.2267 & -0.1060 & 0.4944 & 0.061 \\
\hline C16 & $0.4475(2)$ & $0.29988(14)$ & $0.15040(11)$ & $0.0306(4)$ \\
\hline C17 & $0.4028(2)$ & $0.3261(2)$ & $0.05728(11)$ & $0.0363(4)$ \\
\hline $\mathrm{H} 17 \mathrm{a}$ & 0.4462 & 0.2773 & 0.0153 & 0.054 \\
\hline $\mathrm{H} 17 \mathrm{~b}$ & 0.4221 & 0.4038 & 0.0433 & 0.054 \\
\hline $\mathrm{H} 17 \mathrm{c}$ & 0.3113 & 0.3141 & 0.0533 & 0.054 \\
\hline C18 & $0.4828(2)$ & $0.2420(2)$ & $0.76477(10)$ & $0.0291(4)$ \\
\hline H18 & 0.5474 & 0.2954 & 0.7418 & 0.039 \\
\hline C19 & $0.3716(2)$ & $0.3133(2)$ & $0.80177(13)$ & $0.0428(5)$ \\
\hline $\mathrm{H} 19 \mathrm{a}$ & 0.3360 & 0.3589 & 0.7544 & 0.064 \\
\hline $\mathrm{H} 19 \mathrm{~b}$ & 0.4030 & 0.3617 & 0.8489 & 0.064 \\
\hline $\mathrm{H} 19 \mathrm{c}$ & 0.3059 & 0.2641 & 0.8253 & 0.064 \\
\hline C20 & $0.5477(2)$ & $0.1749(2)$ & $0.83997(12)$ & $0.0388(4)$ \\
\hline $\mathrm{H} 20 \mathrm{a}$ & 0.5837 & 0.2265 & 0.8832 & 0.058 \\
\hline $\mathrm{H} 20 \mathrm{~b}$ & 0.6153 & 0.1287 & 0.8152 & 0.058 \\
\hline $\mathrm{H} 20 \mathrm{c}$ & 0.4851 & 0.1272 & 0.8689 & 0.058 \\
\hline O1 & $0.53713(10)$ & $0.27187(9)$ & $0.49601(7)$ & $0.0279(3)$ \\
\hline
\end{tabular}




\begin{tabular}{|c|c|c|c|c|}
\hline $\mathrm{O} 2$ & $0.27807(11)$ & $0.21458(12)$ & $0.31883(9)$ & $0.0454(3)$ \\
\hline 03 & $0.40305(12)$ & $0.37466(9)$ & $0.21014(8)$ & $0.0316(3)$ \\
\hline O4 & $0.5161(2)$ & $0.22154(12)$ & $0.17152(9)$ & $0.0549(4)$ \\
\hline O5 & $0.27379(12)$ & $0.43998(11)$ & $0.55445(9)$ & $0.0427(3)$ \\
\hline
\end{tabular}

Table S18. Refined Thermal Parameters (U's) for Compound 16

\begin{tabular}{|c|c|c|c|c|c|c|}
\hline Atom & $\mathrm{U}_{11}$ & $\mathrm{U}_{22}$ & $\mathrm{U}_{33}$ & $\mathrm{U}_{23}$ & $\mathrm{U}_{13}$ & $\mathrm{U}_{12}$ \\
\hline C1 & $0.0261(8)$ & $0.0260(9)$ & $0.0387(10)$ & $0.0019(7)$ & $-0.0019(7)$ & $-0.0024(6)$ \\
\hline $\mathrm{C} 2$ & $0.0220(7)$ & $0.0244(8)$ & $0.0268(8)$ & $0.0007(7)$ & $-0.0030(6)$ & $0.0009(6)$ \\
\hline C3 & $0.0218(7)$ & $0.0234(7)$ & $0.0291(8)$ & $0.0014(7)$ & $-0.0012(6)$ & $0.0038(6)$ \\
\hline C4 & $0.0358(8)$ & $0.0257(8)$ & $0.0280(8)$ & $-0.0015(7)$ & $0.0013(7)$ & $0.0026(7)$ \\
\hline C5 & $0.0295(8)$ & $0.0329(9)$ & $0.0194(8)$ & $-0.0018(7)$ & $-0.0026(6)$ & $0.0003(7)$ \\
\hline C6 & $0.0320(8)$ & $0.0290(8)$ & $0.0227(8)$ & $-0.0008(7)$ & $-0.0045(7)$ & $0.0025(7)$ \\
\hline $\mathrm{C7}$ & $0.0536(11)$ & $0.0310(9)$ & $0.0283(9)$ & $-0.0020(8)$ & $-0.0085(8)$ & $0.0114(9)$ \\
\hline C8 & $0.0510(10)$ & $0.0230(9)$ & $0.0274(9)$ & $-0.0013(7)$ & $-0.0059(8)$ & $-0.0041(7)$ \\
\hline C9 & $0.0322(9)$ & $0.0209(7)$ & $0.0278(8)$ & $-0.0033(7)$ & $-0.0052(7)$ & $-0.0035(7)$ \\
\hline C10 & $0.0238(7)$ & $0.0281(8)$ & $0.0211(7)$ & $-0.0018(7)$ & $-0.0008(6)$ & $-0.0008(6)$ \\
\hline C11 & $0.0207(7)$ & $0.0253(8)$ & $0.0243(8)$ & $0.0017(6)$ & $0.0006(6)$ & $0.0011(7)$ \\
\hline C12 & $0.0244(7)$ & $0.0296(9)$ & $0.0245(8)$ & $0.0033(7)$ & $0.0014(6)$ & $0.0034(7)$ \\
\hline C13 & $0.0403(9)$ & $0.0365(10)$ & $0.0345(10)$ & $0.0119(8)$ & $-0.0015(8)$ & $-0.0028(8)$ \\
\hline C14 & $0.0386(9)$ & $0.0290(9)$ & $0.0444(11)$ & $0.0090(8)$ & $-0.0016(8)$ & $-0.0061(8)$ \\
\hline C15 & $0.0405(9)$ & $0.0322(9)$ & $0.0483(11)$ & $-0.0016(9)$ & $-0.0006(9)$ & $-0.0099(8)$ \\
\hline C16 & $0.0361(8)$ & $0.0282(8)$ & $0.0276(9)^{\prime}$ & $0.0000(7)$ & $0.0046(7)$ & $0.0009(7)$ \\
\hline C17 & $0.0478(10)$ & $0.0343(10)$ & $0.0268(9)$ & $-0.0005(7)$ & $0.0012(8)$ & $-0.0014(8)$ \\
\hline C18 & $0.0279(8)$ & $0.0373(9)$ & $0.0220(8)$ & $0.0021(7)$ & $0.0007(6)$ & $0.0003(7)$ \\
\hline C19 & $0.0448(10)$ & $0.0528(12)$ & $0.0308(10)$ & $-0.0021(9)$ & $0.0017(8)$ & $0.0123(9)$ \\
\hline C20 & $0.0354(9)$ & $0.0517(11)$ & $0.0293(9)$ & $0.0039(8)$ & $-0.0035(7)$ & $0.0021(8)$ \\
\hline 01 & $0.0235(5)$ & $0.0310(6)$ & $0.0291(6)$ & $-0.0013(5)$ & $0.0052(4)$ & $-0.0038(4)$ \\
\hline $\mathrm{O} 2$ & $0.0329(7)$ & $0.0591(9)$ & $0.0443(8)$ & $0.0162(7)$ & $-0.0129(6)$ & $-0.0120(6)$ \\
\hline O3 & $0.0438(6)$ & $0.0282(6)$ & $0.0229(6)$ & $0.0010(5)$ & $-0.0025(5)$ & $0.0038(5)$ \\
\hline 04 & $0.0783(10)$ & $0.0530(9)$ & $0.0335(7)$ & $-0.0013(6)$ & $0.0003(7)$ & $0.0298(8)$ \\
\hline O5 & $0.0360(7)$ & $0.0326(7)$ & $0.0596(9)$ & $0.0078(6)$ & $0.0046(6)$ & $0.0062(6)$ \\
\hline
\end{tabular}


Table S19. Bond Distances in Compound 16, $\AA$

\begin{tabular}{|llllll|}
\hline C1-C14 & $1.329(3)$ & C1-C15 & $1.499(2)$ & C1-C2 & $1.518(2)$ \\
C2-C11 & $1.526(2)$ & C2-C3 & $1.539(2)$ & C3-O1 & $1.448(2)$ \\
C3-C4 & $1.528(2)$ & C4-C5 & $1.503(2)$ & C5-O2 & $1.212(2)$ \\
C5-C6 & $1.535(2)$ & C6-O3 & $1.463(2)$ & C6-C7 & $1.524(2)$ \\
C7-C8 & $1.540(2)$ & C8-C9 & $1.512(2)$ & C9-O5 & $1.208(2)$ \\
C9-C10 & $1.527(2)$ & C10-O1 & $1.434(2)$ & C10-C11 & $1.527(2)$ \\
C11-C12 & $1.534(2)$ & C12-C13 & $1.535(2)$ & C12-C18 & $1.540(2)$ \\
C13-C14 & $1.498(3)$ & C16-O4 & $1.206(2)$ & C16-O3 & $1.333(2)$ \\
C16-C17 & $1.492(2)$ & C18-C20 & $1.526(2)$ & C18-C19 & $1.528(2)$ \\
\hline
\end{tabular}

Table S20. Bond Angles in Compound 16,

\begin{tabular}{|llllll|}
\hline C14-C1-C15 & $123.1(2)$ & C14-C1-C2 & $120.4(2)$ & C15-C1-C2 & $116.4(2)$ \\
C1-C2-C11 & $114.34(13)$ & C1-C2-C3 & $115.23(13)$ & C11-C2-C3 & $102.89(12)$ \\
O1-C3-C4 & $106.84(12)$ & O1-C3-C2 & $105.37(12)$ & C4-C3-C2 & $117.59(13)$ \\
C5-C4-C3 & $112.66(13)$ & O2-C5-C4 & $121.8(2)$ & O2-C5-C6 & $118.10(14)$ \\
C4-C5-C6 & $120.12(13)$ & O3-C6-C7 & $103.22(12)$ & O3-C6-C5 & $107.88(12)$ \\
C7-C6-C5 & $114.99(14)$ & C6-C7-C8 & $115.95(14)$ & C9-C8-C7 & $114.59(14)$ \\
O5-C9-C8 & $121.1(2)$ & O5-C9-C10 & $120.8(2)$ & C8-C9-C10 & $118.09(14)$ \\
O1-C10-C11 & $106.83(12)$ & O1-C10-C9 & $108.64(12)$ & C11-C10-C9 & $113.11(12)$ \\
C2-C11-C10 & $100.86(12)$ & C2-C11-C12 & $112.58(12)$ & C10-C11-C12 & $115.00(12)$ \\
C13-C12-C11 & $108.33(13)$ & C13-C12-C18 & $113.69(13)$ & C11-C12-C18 & $113.90(13)$ \\
C14-C13-C12 & $112.8(2)$ & C1-C14-C13 & $125.1(2)$ & O4-C16-O3 & $122.4(2)$ \\
O4-C16-C17 & $125.7(2)$ & O3-C16-C17 & $111.95(14)$ & C20-C18-C19 & $110.70(14)$ \\
C20-C18-C12 & $111.57(14)$ & C19-C18-C12 & $114.08(13)$ & C10-O1-C3 & $109.71(11)$ \\
C16-O3-C6 & $115.96(12)$ & & & & \\
\hline
\end{tabular}




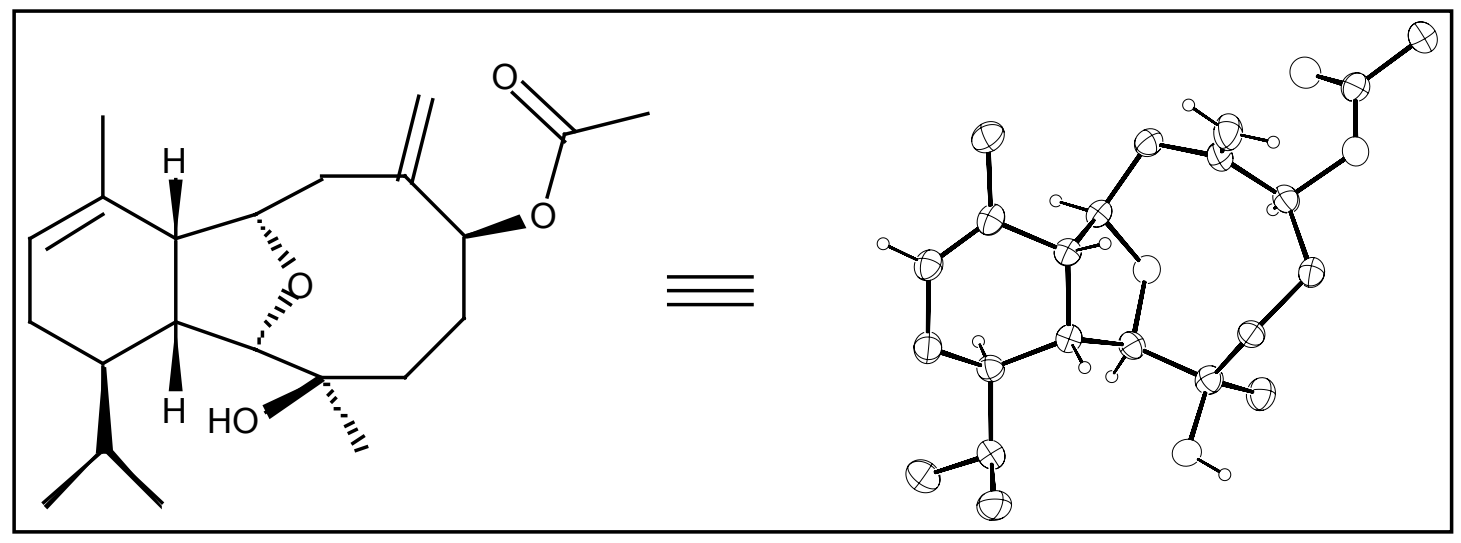

X-ray Structure Determination of Compound 2

Compound 2, $\mathrm{C}_{22} \mathrm{H}_{34} \mathrm{O}_{4}$, crystallizes in the orthorhombic space group $\mathrm{P} 2{ }_{1} 2_{1} 2_{1}$ (systematic absences $\mathrm{h} 00: \mathrm{h}=\mathrm{odd}, 0 \mathrm{k} 0: \mathrm{k}=\mathrm{odd}$, and $001: \mathrm{l}=\mathrm{odd})$ with $\mathrm{a}=8.652(2) \AA, \mathrm{b}=13.943(3) \AA$, $\mathrm{c}=17.078(3) \AA, \mathrm{V}=2060.4(7) \AA^{3}, \mathrm{Z}=4$ and $\mathrm{d}_{\text {calc }}=1.169 \mathrm{~g} / \mathrm{cm}^{3}$. X-ray intensity data were collected on a Rigaku Mercury CCD area detector employing graphite-monochromated Mo- $\mathrm{K}_{\alpha}$ radiation $(\lambda=0.71069 \AA)$ at a temperature of $143^{\circ} \mathrm{K}$. Preliminary indexing was performed from a series of twelve $0.5^{\circ}$ rotation images with exposures of 30 seconds. A total of 440 rotation images were collected with a crystal to detector distance of $36 \mathrm{~mm}$, a $2 \theta$ swing angle of $-10^{\circ}$, rotation widths of $0.5^{\circ}$ and exposures of 90 seconds: scan no. 1 was a $\phi$-scan from $-90^{\circ}$ to $90^{\circ}$ at $\omega=0^{\circ}$ and $\chi=$ $0^{\circ}$ and scan no. 2 was a $\omega$-scan from $-20^{\circ}$ to $20^{\circ}$ at $\phi=0^{\circ}$ and $\chi=-90^{\circ}$. Rotation images were processed using CrystalClear ${ }^{32}$, producing a listing of unaveraged $F^{2}$ and $\sigma\left(F^{2}\right)$ values which were then passed to the CrystalStructure ${ }^{33}$ program package for further processing and structure solution on a Dell Pentium III computer. A total of 11925 reflections were measured over the ranges $5.28 \leq 2 \theta \leq 50.66^{\circ}, \quad-10 \leq \mathrm{h} \leq 8, \quad-16 \leq \mathrm{k} \leq 15, \quad-14 \leq 1 \leq 20$ yielding 3770 unique reflections $\left(\mathrm{R}_{\mathrm{int}}=0.0439\right)$. The intensity data were corrected for Lorentz and polarization effects and for absorption. using REQAB ${ }^{34}$ (minimum and maximum transmission $0.768,1.000$ ).

The structure was solved by direct methods $\left(\operatorname{SIR} 97^{35}\right)$. Refinement was by full-matrix least squares based on $\mathrm{F}^{2}$ using SHELXL-97 ${ }^{36}$. All reflections were used during refinement 
$\left(\mathrm{F}^{2}\right.$ 's that were experimentally negative were replaced by $\mathrm{F}^{2}=0$ ). The weighting scheme used was $\mathrm{w}=1 /\left[\sigma^{2}\left(\mathrm{~F}_{\mathrm{o}}^{2}\right)+0.0921 \mathrm{P}^{2}+0.8109 \mathrm{P}\right]$ where $\mathrm{P}=\left(\mathrm{F}_{\mathrm{o}}^{2}+2 \mathrm{~F}_{\mathrm{c}}^{2}\right) / 3$. Non-hydrogen atoms were refined anisotropically and hydrogen atoms were refined using a "riding" model. Refinement converged to $\mathrm{R}_{1}=0.0656$ and $\mathrm{wR}_{2}=0.1636$ for 3110 reflections for which $\mathrm{F}>4 \sigma(\mathrm{F})$ and $\mathrm{R}_{1}=0.0787, \mathrm{wR}_{2}=0.1764$ and $\mathrm{GOF}=1.064$ for all 3770 unique, non-zero reflections and 242 variables $^{37}$. The maximum $\Delta / \sigma$ in the final cycle of least squares was 0.000 and the two most prominent peaks in the final difference Fourier were +0.225 and $-0.219 \mathrm{e} / \AA^{3}$.

Table S21. lists cell information, data collection parameters, and refinement data. Final positional and equivalent isotropic thermal parameters are given in Table S22. Anisotropic thermal parameters are in Table S23. Tables S24. and S25. list bond distances and bond angles. Figure 1. is an $\mathrm{ORTEP}^{38}$ representation of the molecule with $30 \%$ probability thermal ellipsoids displayed.

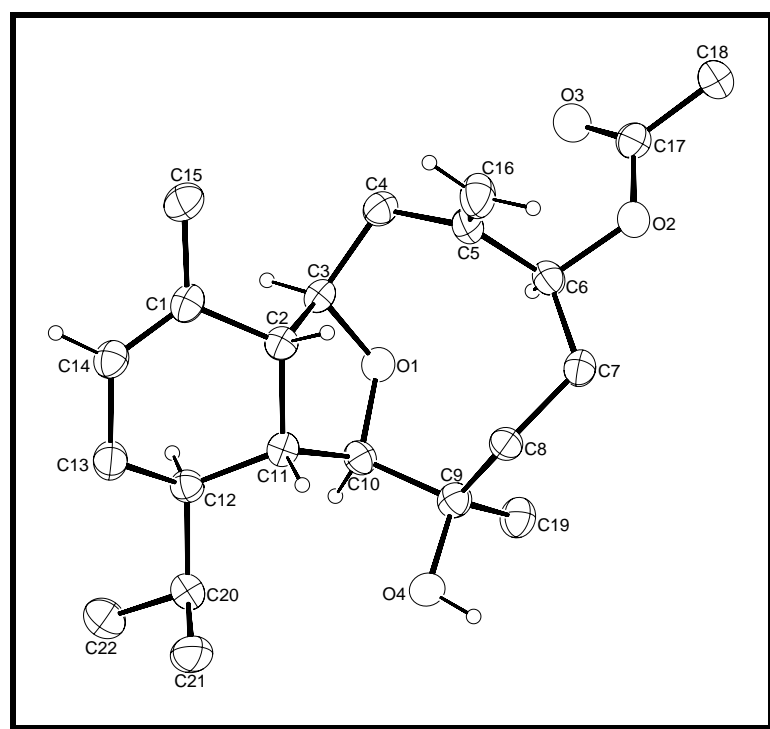

Figure S6. ORTEP drawing of the title compound with $30 \%$ probability thermal ellipsoids. 


\section{Table S21. Summary of Structure Determination of Compound 2}

Formula:

Formula weight:

Crystal class:

Space group:

Z

Cell constants:

a

$\mathrm{b}$

c

$\mathrm{V}$

$\mu$

crystal size, $\mathrm{mm}$

$\mathrm{D}_{\text {calc }}$

$\mathrm{F}(000)$

Radiation:

$2 \theta$ range

hkl collected:

No. reflections measured:

No. unique reflections:

No. observed reflections

No. reflections used in refinement

No. parameters

$\mathrm{R}$ indices $(\mathrm{F}>4 \sigma)$

$\mathrm{R}$ indices (all data)

GOF:

Final Difference Peaks, e/ $\AA^{3}$
$\mathrm{C}_{22} \mathrm{H}_{34} \mathrm{O}_{4}$

362.49

orthorhombic

$\mathrm{P} 2{ }_{1} 22_{1}$ (\#19)

4

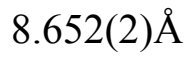

$13.943(3) \AA$

$17.078(3) \AA$

2060.4(7) $\AA^{3}$

$0.78 \mathrm{~cm}^{-1}$

$0.32 \times 0.15 \times 0.04$

$1.169 \mathrm{~g} / \mathrm{cm}^{3}$

792

$\mathrm{Mo}-\mathrm{K}_{\alpha}(\lambda=0.71069 \AA)$

$5.28-50.66^{\circ}$

$-10 \leq \mathrm{h} \leq 8 ;-16 \leq \mathrm{k} \leq 15 ;-14 \leq 1 \leq 20$

11925

$3770\left(\mathrm{R}_{\mathrm{int}}=0.0439\right)$

$3110(\mathrm{~F}>4 \sigma)$

3770

242

$\mathrm{R}_{1}=0.0656$

$\mathrm{wR}_{2}=0.1636$

$\mathrm{R}_{1}=0.0787$

$\mathrm{wR}_{2}=0.1764$

1.064

$+0.225,-0.219$ 
Table S22. Refined Positional Parameters for Compound 2

\begin{tabular}{|c|c|c|c|c|}
\hline Atom & $\mathrm{X}$ & $\mathrm{y}$ & $\mathrm{Z}$ & $\mathrm{U}_{\mathrm{eq}}, \AA^{2}$ \\
\hline C1 & $0.6531(4)$ & $0.4767(2)$ & $0.5595(2)$ & $0.0421(8)$ \\
\hline C2 & $0.6051(4)$ & $0.4424(2)$ & $0.4788(2)$ & $0.0370(7)$ \\
\hline $\mathrm{H} 2$ & 0.5907 & 0.3728 & 0.4816 & 0.049 \\
\hline C3 & $0.7272(4)$ & $0.4626(2)$ & $0.4151(2)$ & $0.0386(7)$ \\
\hline $\mathrm{H} 3$ & 0.7780 & 0.5239 & 0.4263 & 0.051 \\
\hline C4 & $0.8503(4)$ & $0.3853(3)$ & $0.4032(2)$ & $0.0455(8)$ \\
\hline $\mathrm{H} 4 \mathrm{a}$ & 0.9218 & 0.4067 & 0.3631 & 0.060 \\
\hline $\mathrm{H} 4 \mathrm{~b}$ & 0.9082 & 0.3780 & 0.4515 & 0.060 \\
\hline C5 & $0.7866(4)$ & $0.2895(2)$ & $0.3799(2)$ & $0.0436(8)$ \\
\hline C6 & $0.7296(4)$ & $0.2784(2)$ & $0.2960(2)$ & $0.0403(7)$ \\
\hline H6 & 0.7478 & 0.3378 & 0.2669 & 0.054 \\
\hline C7 & $0.5611(4)$ & $0.2481(2)$ & $0.2877(2)$ & $0.0434(8)$ \\
\hline $\mathrm{H} 7 \mathrm{a}$ & 0.5541 & 0.1814 & 0.3036 & 0.058 \\
\hline $\mathrm{H} 7 \mathrm{~b}$ & 0.5357 & 0.2504 & 0.2324 & 0.058 \\
\hline C8 & $0.4333(4)$ & $0.3035(2)$ & $0.3319(2)$ & $0.0385(7)$ \\
\hline $\mathrm{H} 8 \mathrm{a}$ & 0.3390 & 0.2663 & 0.3274 & 0.051 \\
\hline $\mathrm{H} 8 \mathrm{~b}$ & 0.4614 & 0.3037 & 0.3869 & 0.051 \\
\hline C9 & $0.3932(4)$ & $0.4076(2)$ & $0.3095(2)$ & $0.0398(8)$ \\
\hline C10 & $0.4812(3)$ & $0.4847(2)$ & $0.3556(2)$ & $0.0365(7)$ \\
\hline $\mathrm{H} 10$ & 0.4527 & 0.5477 & 0.3346 & 0.049 \\
\hline C11 & $0.4551(4)$ & $0.4860(2)$ & $0.4453(2)$ & $0.0352(7)$ \\
\hline $\mathrm{H} 11$ & 0.3678 & 0.4442 & 0.4583 & 0.047 \\
\hline C12 & $0.4227(3)$ & $0.5875(2)$ & $0.4763(2)$ & $0.0382(7)$ \\
\hline $\mathrm{H} 12$ & 0.5080 & 0.6286 & 0.4589 & 0.051 \\
\hline C13 & $0.4252(4)$ & $0.5870(3)$ & $0.5662(2)$ & $0.0479(8)$ \\
\hline H13a & 0.4188 & 0.6524 & 0.5851 & 0.064 \\
\hline $\mathrm{H} 13 \mathrm{~b}$ & 0.3353 & 0.5526 & 0.5853 & 0.064 \\
\hline C14 & $0.5670(4)$ & $0.5413(2)$ & $0.5982(2)$ & $0.0445(8)$ \\
\hline $\mathrm{H} 14$ & 0.5982 & 0.5587 & 0.6484 & 0.059 \\
\hline C15 & $0.7946(4)$ & $0.4332(3)$ & $0.5954(2)$ & $0.0565(10)$ \\
\hline $\mathrm{H} 15 \mathrm{a}$ & 0.8029 & 0.4533 & 0.6490 & 0.085 \\
\hline $\mathrm{H} 15 \mathrm{~b}$ & 0.8844 & 0.4537 & 0.5670 & 0.085 \\
\hline $\mathrm{H} 15 \mathrm{c}$ & 0.7871 & 0.3645 & 0.5933 & 0.085 \\
\hline C16 & $0.7830(5)$ & $0.2147(3)$ & $0.4281(2)$ & $0.0633(11)$ \\
\hline $\mathrm{H} 16 \mathrm{a}$ & 0.8211 & 0.2205 & 0.4788 & 0.084 \\
\hline $\mathrm{H} 16 \mathrm{~b}$ & 0.7424 & 0.1566 & 0.4111 & 0.084 \\
\hline C17 & $0.9648(4)$ & $0.2130(3)$ & $0.2440(2)$ & $0.0460(8)$ \\
\hline C18 & $1.0422(5)$ & $0.1225(3)$ & $0.2188(3)$ & $0.0634(11)$ \\
\hline $\mathrm{H} 18 \mathrm{a}$ & 1.1246 & 0.1374 & 0.1832 & 0.095 \\
\hline $\mathrm{H} 18 \mathrm{~b}$ & 0.9684 & 0.0817 & 0.1932 & 0.095 \\
\hline $\mathrm{H} 18 \mathrm{c}$ & 1.0834 & 0.0901 & 0.2639 & 0.095 \\
\hline C19 & $0.4115(5)$ & $0.4248(3)$ & $0.2214(2)$ & $0.0500(9)$ \\
\hline $\mathrm{H} 19 \mathrm{a}$ & 0.3532 & 0.3776 & 0.1931 & 0.075 \\
\hline $\mathrm{H} 19 \mathrm{~b}$ & 0.5186 & 0.4199 & 0.2074 & 0.075 \\
\hline $\mathrm{H} 19 \mathrm{c}$ & 0.3741 & 0.4877 & 0.2086 & 0.075 \\
\hline C20 & $0.2723(4)$ & $0.6313(2)$ & $0.4442(2)$ & $0.0484(9)$ \\
\hline $\mathrm{H} 20$ & 0.2707 & 0.6186 & 0.3877 & 0.064 \\
\hline C21 & $0.1256(4)$ & $0.5867(4)$ & $0.4785(3)$ & $0.0651(11)$ \\
\hline $\mathrm{H} 21 \mathrm{a}$ & 0.1274 & 0.5186 & 0.4703 & 0.098 \\
\hline
\end{tabular}




\begin{tabular}{|lllll|}
$\mathrm{H} 21 \mathrm{~b}$ & 0.0367 & 0.6137 & 0.4531 & 0.098 \\
$\mathrm{H} 21 \mathrm{c}$ & 0.1209 & 0.6000 & 0.5336 & 0.098 \\
$\mathrm{C} 22$ & $0.2709(6)$ & $0.7400(3)$ & $0.4543(3)$ & $0.0726(13)$ \\
$\mathrm{H} 22 \mathrm{a}$ & 0.1791 & 0.7659 & 0.4309 & 0.109 \\
$\mathrm{H} 22 \mathrm{~b}$ & 0.3602 & 0.7670 & 0.4293 & 0.109 \\
$\mathrm{H} 22 \mathrm{c}$ & 0.2725 & 0.7554 & 0.5091 & 0.109 \\
$\mathrm{O} 1$ & $0.6443(2)$ & $0.4703(2)$ & $0.34265(12)$ & $0.0389(5)$ \\
$\mathrm{O} 2$ & $0.8129(2)$ & $0.1987(2)$ & $0.2587(2)$ & $0.0462(6)$ \\
$\mathrm{O} 3$ & $1.0272(3)$ & $0.2897(2)$ & $0.2526(2)$ & $0.0568(7)$ \\
$\mathrm{O} 4$ & $0.2348(3)$ & $0.4260(2)$ & $0.3300(2)$ & $0.0478(6)$ \\
$\mathrm{H} 4$ & 0.1794 & 0.3846 & 0.3106 & 0.072 \\
\hline \multicolumn{2}{r}{$\mathrm{U}_{\mathrm{eq}}=1 / 3\left[\mathrm{U}_{11}(\mathrm{aa})^{2}+\mathrm{U}_{22}(\mathrm{bb})^{2}+\mathrm{U}_{33}(\mathrm{cc} *)^{2}+2 \mathrm{U}_{12} \mathrm{aa} * \mathrm{bb} * \cos \gamma+2 \mathrm{U}_{13} \mathrm{aa} * \mathrm{cc} * \cos \beta+2 \mathrm{U}_{23} \mathrm{bb}{ }^{*} \mathrm{cc} * c\right.$} \\
\hline
\end{tabular}

Table S23. Refined Thermal Parameters (U's) for Compound 2

\begin{tabular}{|c|c|c|c|c|c|c|}
\hline Atom & $\mathrm{U}_{11}$ & $\mathrm{U}_{22}$ & $\mathrm{U}_{33}$ & $\mathrm{U}_{23}$ & $\mathrm{U}_{13}$ & $\mathrm{U}_{12}$ \\
\hline C1 & $0.051(2)$ & $0.038(2)$ & $0.038(2)$ & $0.002(2)$ & $-0.0055(14)$ & $-0.0047(14)$ \\
\hline $\mathrm{C} 2$ & $0.039(2)$ & $0.036(2)$ & $0.036(2)$ & $0.0011(13)$ & $-0.0006(13)$ & $-0.0008(12)$ \\
\hline C3 & $0.037(2)$ & $0.040(2)$ & $0.038(2)$ & $-0.0050(14)$ & $-0.0056(13)$ & $-0.0035(13)$ \\
\hline C4 & $0.039(2)$ & $0.051(2)$ & $0.046(2)$ & $-0.008(2)$ & $-0.002(2)$ & $-0.0040(14)$ \\
\hline C5 & $0.048(2)$ & $0.038(2)$ & $0.045(2)$ & $-0.007(2)$ & $-0.0026(14)$ & $0.008(2)$ \\
\hline C6 & $0.042(2)$ & $0.036(2)$ & $0.043(2)$ & $-0.0034(14)$ & $0.0008(14)$ & $0.0047(14)$ \\
\hline C7 & $0.043(2)$ & $0.042(2)$ & $0.045(2)$ & $-0.008(2)$ & $-0.0019(14)$ & $-0.0021(14)$ \\
\hline C8 & $0.035(2)$ & $0.042(2)$ & $0.038(2)$ & $-0.0007(14)$ & $0.0019(13)$ & $-0.0010(13)$ \\
\hline C9 & $0.039(2)$ & $0.046(2)$ & $0.035(2)$ & $0.0005(14)$ & $-0.0002(13)$ & $-0.0013(14)$ \\
\hline C10 & $0.038(2)$ & $0.034(2)$ & $0.038(2)$ & $0.0027(14)$ & $0.0018(13)$ & $0.0018(12)$ \\
\hline C11 & $0.038(2)$ & $0.034(2)$ & $0.033(2)$ & $0.0012(13)$ & $0.0007(12)$ & $-0.0041(12)$ \\
\hline C12 & $0.034(2)$ & $0.040(2)$ & $0.041(2)$ & $-0.0009(14)$ & $0.0028(13)$ & $-0.0011(13)$ \\
\hline C13 & $0.052(2)$ & $0.047(2)$ & $0.044(2)$ & $-0.007(2)$ & $-0.003(2)$ & $-0.001(2)$ \\
\hline C14 & $0.051(2)$ & $0.044(2)$ & $0.038(2)$ & $0.001(2)$ & $-0.006(2)$ & $-0.002(2)$ \\
\hline C15 & $0.058(2)$ & $0.067(2)$ & $0.045(2)$ & $-0.001(2)$ & $-0.014(2)$ & $0.007(2)$ \\
\hline C16 & $0.091(3)$ & $0.046(2)$ & $0.053(2)$ & $-0.006(2)$ & $-0.019(2)$ & $0.012(2)$ \\
\hline C17 & $0.045(2)$ & $0.054(2)$ & $0.039(2)$ & $-0.005(2)$ & $-0.005(2)$ & $0.003(2)$ \\
\hline C18 & $0.057(2)$ & $0.067(3)$ & $0.067(3)$ & $-0.021(2)$ & $-0.006(2)$ & $0.018(2)$ \\
\hline C19 & $0.059(2)$ & $0.052(2)$ & $0.039(2)$ & $-0.001(2)$ & $-0.001(2)$ & $-0.001(2)$ \\
\hline C20 & $0.045(2)$ & $0.047(2)$ & $0.053(2)$ & $-0.011(2)$ & $-0.002(2)$ & $0.008(2)$ \\
\hline $\mathrm{C} 21$ & $0.040(2)$ & $0.093(3)$ & $0.063(3)$ & $-0.016(2)$ & $0.000(2)$ & $0.003(2)$ \\
\hline C22 & $0.072(3)$ & $0.057(2)$ & $0.089(3)$ & $-0.012(2)$ & $-0.015(2)$ & $0.023(2)$ \\
\hline 01 & $0.0360(12)$ & $0.0471(13)$ & $0.0337(11)$ & $-0.0013(10)$ & $0.0041(9)$ & $-0.0032(9)$ \\
\hline $\mathrm{O} 2$ & $0.0430(13)$ & $0.0444(13)$ & $0.0513(14)$ & $-0.0114(12)$ & $-0.0013(10)$ & $0.0019(9)$ \\
\hline O3 & $0.0458(14)$ & $0.064(2)$ & $0.061(2)$ & $-0.0128(14)$ & $0.0030(12)$ & $-0.0011(12)$ \\
\hline O4 & $0.0338(12)$ & $0.053(2)$ & $0.057(2)$ & $-0.0098(12)$ & $-0.0042(10)$ & $-0.0008(10)$ \\
\hline
\end{tabular}


Table S24. Bond Distances in Compound 2, $\AA$

\begin{tabular}{|llllll|}
\hline C1-C14 & $1.344(5)$ & C1-C15 & $1.498(5)$ & C1-C2 & $1.516(5)$ \\
C2-C11 & $1.544(4)$ & C2-C3 & $1.543(4)$ & C3-O1 & $1.434(4)$ \\
C3-C4 & $1.528(5)$ & C4-C5 & $1.500(5)$ & C5-C16 & $1.328(5)$ \\
C5-C6 & $1.523(5)$ & C6-O2 & $1.469(4)$ & C6-C7 & $1.525(5)$ \\
C7-C8 & $1.546(5)$ & C8-C9 & $1.540(5)$ & C9-O4 & $1.438(4)$ \\
C9-C19 & $1.531(5)$ & C9-C10 & $1.535(5)$ & C10-O1 & $1.443(4)$ \\
C10-C11 & $1.547(4)$ & C11-C12 & $1.536(4)$ & C12-C13 & $1.536(5)$ \\
C12-C20 & $1.539(5)$ & C13-C14 & $1.486(5)$ & C17-O3 & $1.208(4)$ \\
C17-O2 & $1.353(4)$ & C17-C18 & $1.492(5)$ & C20-C22 & $1.525(5)$ \\
C20-C21 & $1.530(6)$ & & & & \\
\hline
\end{tabular}

Table S25. Bond Angles in Compound 2,

\begin{tabular}{|llllll|}
\hline C14-C1-C15 & $121.5(3)$ & C14-C1-C2 & $120.5(3)$ & C15-C1-C2 & $118.0(3)$ \\
C1-C2-C11 & $116.3(3)$ & C1-C2-C3 & $113.3(3)$ & C11-C2-C3 & $104.0(2)$ \\
O1-C3-C4 & $106.7(3)$ & O1-C3-C2 & $106.2(2)$ & C4-C3-C2 & $116.3(3)$ \\
C5-C4-C3 & $114.0(3)$ & C16-C5-C4 & $122.9(3)$ & C16-C5-C6 & $119.7(3)$ \\
C4-C5-C6 & $117.4(3)$ & O2-C6-C5 & $109.1(3)$ & O2-C6-C7 & $102.7(2)$ \\
C5-C6-C7 & $115.2(3)$ & C6-C7-C8 & $120.0(3)$ & C9-C8-C7 & $120.7(3)$ \\
O4-C9-C19 & $108.0(3)$ & O4-C9-C10 & $102.9(3)$ & C19-C9-C10 & $110.1(3)$ \\
O4-C9-C8 & $108.8(3)$ & C19-C9-C8 & $111.7(3)$ & C10-C9-C8 & $114.9(3)$ \\
O1-C10-C9 & $108.0(3)$ & O1-C10-C11 & $107.2(2)$ & C9-C10-C11 & $116.3(3)$ \\
C12-C11-C2 & $112.8(2)$ & C12-C11-C10 & $112.3(3)$ & C2-C11-C10 & $103.9(2)$ \\
C13-C12-C11 & $109.8(3)$ & C13-C12-C20 & $111.7(3)$ & C11-C12-C20 & $113.4(3)$ \\
C14-C13-C12 & $112.4(3)$ & C1-C14-C13 & $124.3(3)$ & O3-C17-O2 & $122.8(3)$ \\
O3-C17-C18 & $125.7(3)$ & O2-C17-C18 & $111.4(3)$ & C22-C20-C21 & $110.7(3)$ \\
C22-C20-C12 & $111.2(3)$ & C21-C20-C12 & $113.8(3)$ & C3-O1-C10 & $111.5(2)$ \\
C17-O2-C6 & $116.5(3)$ & & & & \\
\hline
\end{tabular}

\section{References}

${ }^{1}$ Molander, G. A.; Jeffery, S. C. Tetrahedron Lett. 2002, 43, 359-362.

${ }^{2}$ The neat compound exits exclusively in the enol tautomer but upon standing in solution a mixture of both the keto and enol forms are obtained.

${ }^{3}$ Uchio, Y.; Kodama, M.; Usui, S.; Fukazawa, Y. Tetrahedron 1992, 33, 1317-1320.

${ }^{4}$ MacMillan, D. W. C.; Overman, L. E. J. Am. Chem. Soc. 1995, 117, 10391-10392.

5 . bioteX: A suite of Programs for the Collection, Reduction and Interpretation of Imaging Plate

Data, Molecular Structure Corporation (1995). 
${ }^{6}$. teXsan: Crystal Structure Analysis Package, Molecular Structure Corporation (1985 \& 1992).

7. SIR92: Altomare, A., Burla, M.C., Camalli, M., Cascarano, M., Giacovazzo, C., Guagliardi, A., Polidoro, G. (1994). J. Appl. Cryst., 27, 435.

${ }^{8}$. SHELXL-93: Program for the Refinement of Crystal Structures, Sheldrick, G.M. (1993), University of Göttingen, Germany.

9. $\mathrm{R}_{1}=\sum|| \mathrm{F}_{\mathrm{o}}|-| \mathrm{F}_{\mathrm{c}}\left|/ \sum\right| \mathrm{F}_{\mathrm{o}} \mid$

$\mathrm{wR}_{2}=\left\{\sum \mathrm{w}\left(\mathrm{F}_{\mathrm{o}}^{2}-\mathrm{F}_{\mathrm{c}}^{2}\right)^{2} / \sum \mathrm{w}\left(\mathrm{F}_{\mathrm{o}}^{2}\right)^{2}\right\}^{1 / 2}$

$\mathrm{GOF}=\left\{\sum \mathrm{w}\left(\mathrm{F}_{\mathrm{o}}^{2}-\mathrm{F}_{\mathrm{c}}^{2}\right)^{2} /(\mathrm{n}-\mathrm{p})\right\}^{1 / 2}$

where $\mathrm{n}=$ the number of reflections and $\mathrm{p}=$ the number of parameters refined.

10. "ORTEP-II: A Fortran Thermal Ellipsoid Plot Program for Crystal Structure Illustrations". C.K. Johnson (1976) ORNL-5138.

${ }^{11}$. CrystalClear: Rigaku Corporation, 1999.

12. CrystalStructure: Crystal Structure Analysis Package, Rigaku Corp. \% Rigaku/MSC (2002).

13. REQAB4: R.A. Jacobsen, (1994). Private Communication.

${ }^{14}$. SIR97: Altomare, A., M. Burla, M. Camalli, G. Cascarano, C. Giacovazzo, A. Guagliardi, A. Moliterni, G. Polidori \& R. Spagna (1999). J. Appl. Cryst., 32, 115-119.

${ }^{15}$. SHELXL-97: Program for the Refinement of Crystal Structures, Sheldrick, G.M. (1997), University of Göttingen, Germany.

${ }^{16} . \mathrm{R}_{1}=\sum|| \mathrm{F}_{\mathrm{o}}|-| \mathrm{F}_{\mathrm{c}}\left|/ / \sum\right| \mathrm{F}_{\mathrm{o}} \mid$

$\mathrm{wR}_{2}=\left\{\sum \mathrm{w}\left(\mathrm{F}_{\mathrm{o}}^{2}-\mathrm{F}_{\mathrm{c}}^{2}\right)^{2} / \sum \mathrm{w}\left(\mathrm{F}_{\mathrm{o}}^{2}\right)^{2}\right\}^{1 / 2}$

$\mathrm{GOF}=\left\{\sum \mathrm{w}\left(\mathrm{F}_{\mathrm{o}}^{2}-\mathrm{F}_{\mathrm{c}}^{2}\right)^{2} /(\mathrm{n}-\mathrm{p})\right\}^{1 / 2}$

where $n=$ the number of reflections and $\mathrm{p}=$ the number of parameters refined.

17. "ORTEP-II: A Fortran Thermal Ellipsoid Plot Program for Crystal Structure Illustrations". C.K. Johnson (1976) ORNL-5138.

${ }^{18}$. CrystalClear: Rigaku Corporation, 1999.

${ }^{19}$. CrystalStructure: Crystal Structure Analysis Package, Rigaku Corp. \% Rigaku/MSC (2002).

${ }^{20}$. REQAB4: R.A. Jacobsen, (1994). Private Communication.

${ }^{21}$. SIR97: Altomare, A., M. Burla, M. Camalli, G. Cascarano, C. Giacovazzo, A. Guagliardi, A. Moliterni, G. Polidori \& R. Spagna (1999). J. Appl. Cryst., 32, 115-119. 
22. SHELXL-97: Program for the Refinement of Crystal Structures, Sheldrick, G.M. (1997), University of Göttingen, Germany.

${ }^{23} \cdot \mathrm{R}_{1}=\sum|| \mathrm{F}_{\mathrm{o}}|-| \mathrm{F}_{\mathrm{c}}\left|/ / \sum\right| \mathrm{F}_{\mathrm{o}} \mid$

$\mathrm{wR}_{2}=\left\{\sum \mathrm{w}\left(\mathrm{F}_{\mathrm{o}}^{2}-\mathrm{F}_{\mathrm{c}}^{2}\right)^{2} / \sum \mathrm{w}\left(\mathrm{F}_{\mathrm{o}}^{2}\right)^{2}\right\}^{1 / 2}$

$\mathrm{GOF}=\left\{\sum \mathrm{w}\left(\mathrm{F}_{\mathrm{o}}^{2}-\mathrm{F}_{\mathrm{c}}^{2}\right)^{2} /(\mathrm{n}-\mathrm{p})\right\}^{1 / 2}$

where $\mathrm{n}=$ the number of reflections and $\mathrm{p}=$ the number of parameters refined.

24 . "ORTEP-II: A Fortran Thermal Ellipsoid Plot Program for Crystal Structure Illustrations". C.K. Johnson (1976) ORNL-5138.

${ }^{25}$. CrystalClear: Rigaku Corporation, 1999.

${ }^{26}$. CrystalStructure: Crystal Structure Analysis Package, Rigaku Corp. \% Rigaku/MSC (2002).

${ }^{27}$. REQAB4: R.A. Jacobsen, (1994). Private Communication.

${ }^{28}$ SIR97: Altomare, A., M. Burla, M. Camalli, G. Cascarano, C. Giacovazzo, A. Guagliardi, A. Moliterni, G. Polidori \& R. Spagna (1999). J. Appl. Cryst., 32, 115-119.

29. SHELXL-97: Program for the Refinement of Crystal Structures, Sheldrick, G.M. (1997), University of Göttingen, Germany.

${ }^{30} \cdot \mathrm{R}_{1}=\sum|| \mathrm{F}_{\mathrm{o}}|-| \mathrm{F}_{\mathrm{c}}\left|/ / \sum\right| \mathrm{F}_{\mathrm{o}} \mid$

$\mathrm{wR}_{2}=\left\{\sum \mathrm{w}\left(\mathrm{F}_{\mathrm{o}}^{2}-\mathrm{F}_{\mathrm{c}}^{2}\right)^{2} / \sum \mathrm{w}\left(\mathrm{F}_{\mathrm{o}}^{2}\right)^{2}\right\}^{1 / 2}$

$\mathrm{GOF}=\left\{\sum \mathrm{w}\left(\mathrm{F}_{\mathrm{o}}^{2}-\mathrm{F}_{\mathrm{c}}^{2}\right)^{2} /(\mathrm{n}-\mathrm{p})\right\}^{1 / 2}$

where $\mathrm{n}=$ the number of reflections and $\mathrm{p}=$ the number of parameters refined.

31 . "ORTEP-II: A Fortran Thermal Ellipsoid Plot Program for Crystal Structure Illustrations". C.K. Johnson (1976) ORNL-5138.

32. CrystalClear: Rigaku Corporation, 1999.

${ }^{33}$. CrystalStructure: Crystal Structure Analysis Package, Rigaku Corp. Rigaku/MSC (2002).

${ }^{34}$. REQAB4: R.A. Jacobsen, (1994). Private Communication.

${ }^{35}$. SIR97: Altomare, A., M. Burla, M. Camalli, G. Cascarano, C. Giacovazzo, A. Guagliardi, A. Moliterni, G. Polidori \& R. Spagna (1999). J. Appl. Cryst., 32, 115-119.

36. SHELXL-97: Program for the Refinement of Crystal Structures, Sheldrick, G.M. (1997), University of Göttingen, Germany.

37. $\mathrm{R}_{1}=\sum|| \mathrm{F}_{\mathrm{o}}|-| \mathrm{F}_{\mathrm{c}}\left|/ / \sum\right| \mathrm{F}_{\mathrm{o}} \mid$ 


$$
\begin{aligned}
& \mathrm{wR}_{2}=\left\{\sum \mathrm{w}\left(\mathrm{F}_{\mathrm{o}}^{2}-\mathrm{F}_{\mathrm{c}}^{2}\right)^{2} / \sum \mathrm{w}\left(\mathrm{F}_{\mathrm{o}}^{2}\right)^{2}\right\}^{1 / 2} \\
& \mathrm{GOF}=\left\{\sum \mathrm{w}\left(\mathrm{F}_{\mathrm{o}}^{2}-\mathrm{F}_{\mathrm{c}}^{2}\right)^{2} /(\mathrm{n}-\mathrm{p})\right\}^{1 / 2}
\end{aligned}
$$

where $\mathrm{n}=$ the number of reflections and $\mathrm{p}=$ the number of parameters refined.

38 . "ORTEP-II: A Fortran Thermal Ellipsoid Plot Program for Crystal Structure Illustrations”. C.K. Johnson (1976) ORNL-5138. 